GA-A21093

UC-712

\title{
CONCEPTUAL DESIGN REPORT FOR THE UNIVERSITY OF ROCHESTER CRYOGENIC TARGET DELIVERY SYSTEM
}

by

R.L. FAGALY, N.B. ALEXANDER, D.N. BITTNER, ${ }^{*}$ R.F. BOURQUE, C.F. DAHMS, C.D. HENDRICKS, ${ }^{*}$ J.R. LINDGREN, and W.J. MILLER

Prepared under

Contract No. DE-AC03-91SF18601 for the U.S. Department of Energy

*W.J. Schafer Associates, Livermore, CA.

GENERAL ATOMICS PROJECT 3896 DATE PUBLISHED: MAY 1993

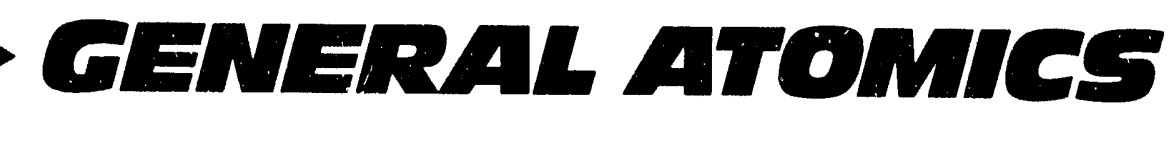




\section{DISCLAIMER}

This report was prepared as an account of work sponsored by an agency of the United States Government. Neither the United States Government nor any agency thereof, nor any of their employees, maloes any warranty, express or implied, or assumes any legal liability or responsibility for the accuracy, completeness, or usefulness of any information, apparatus, product, or process disclosed, or represents that its use would not infringe privately owned rights. Reference herein to any specific commercial product, process, or service by trade name, trademark, manufacturer, or otherwise, does not necessarily constitute or imply its endorsement, recommendation, or favoring by the United States Government or any agency thereof. The views and opinions of authors expressed herein do not necessarily state or reflect those of the United States Government or any agency thereof.

This report has been reproduced directly from the best available copy.

Available to DOE and DOE contractors from the

Office of Scientific and Technical Information

P.O. Box 62

Oak Ridge, TN 37831

Prices available from (615) 576-8401, FTS 626-8401

Available to the public from the

National Technical Information Service

U.S. Department of Commerce

5285 Port Royal Road

Springfield, VA 22161 


\section{CONTENTS}

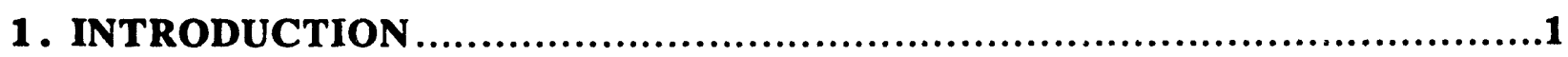

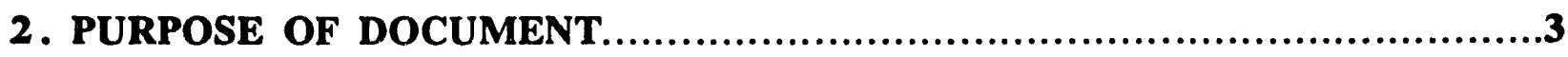

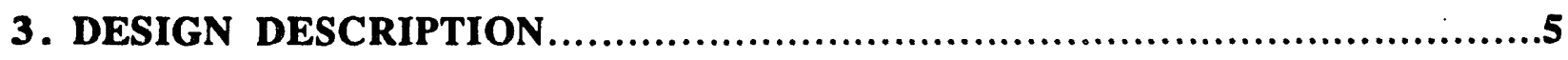

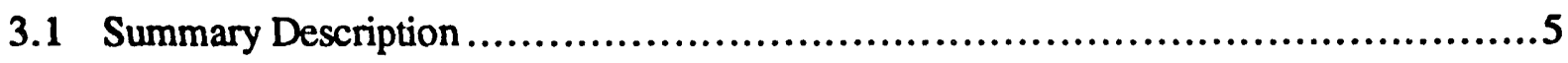

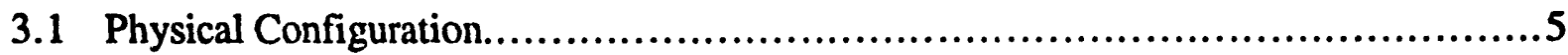

3.3 Integration with the rest of the Omega Upgrade program......................................6

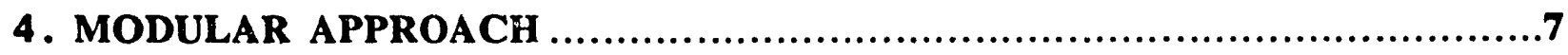

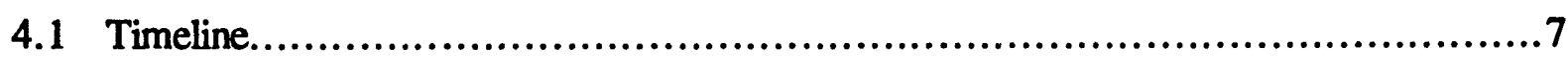

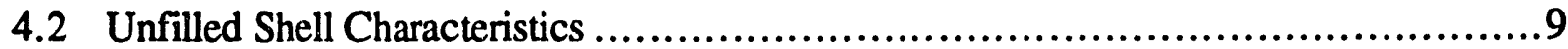

4.3 Target Support................................................................... 11

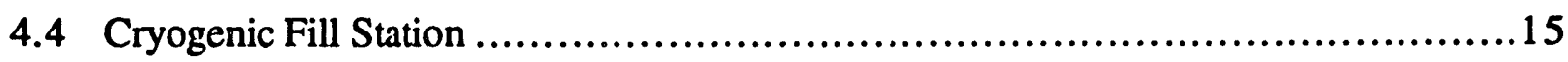

4.5 Transport to Storage Cryostat ..................................................27

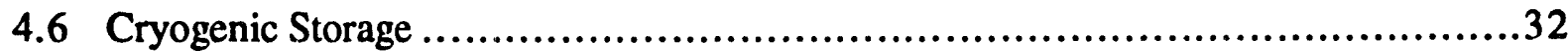

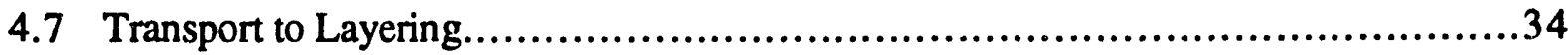

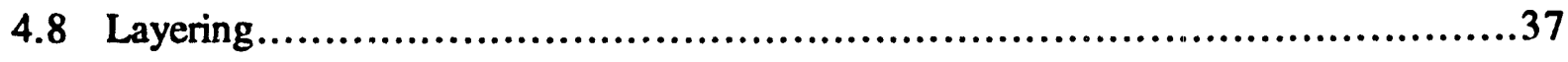

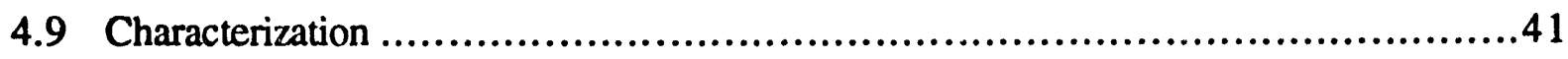

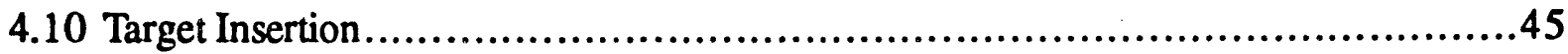

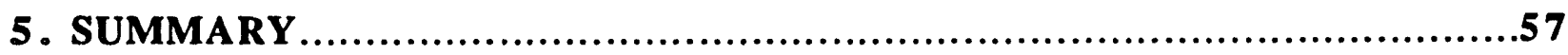

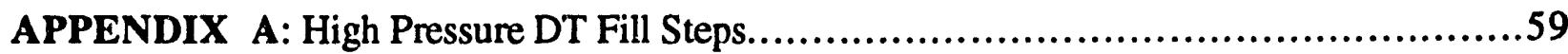

APPENDIX B: Description of Transport Cryogenic Storage Modules..........................61

APPENDIX C: Description of Transport to the Layering Module.............................79

ATTACHMENT A: Design Requirements Report ........................................95 


\section{FIGURES}

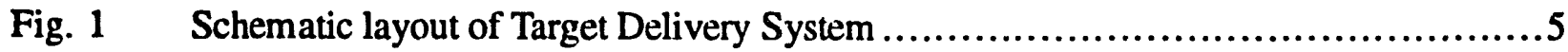

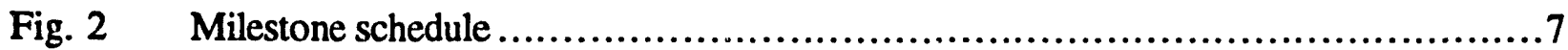

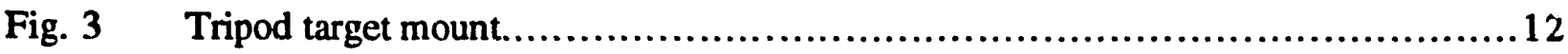

Fig. $4 \quad$ Laser beam paths and target support structure.................................... 13

Fig. 5 Block diagram of existing UR/LLE tritium fill system ......................... 18

Fig. 6 Block diagram of the high pressure fill system.......................................19

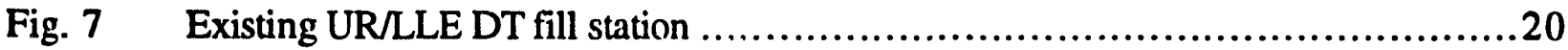

Fig. 8 Major components of the high pressure cryogenic fill station .......................21

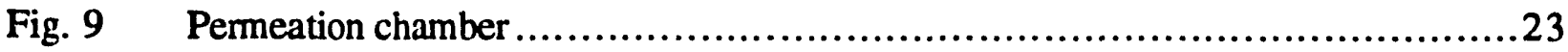

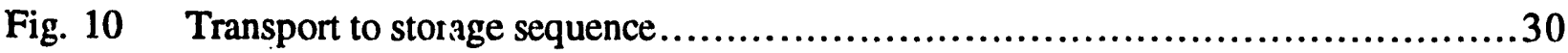

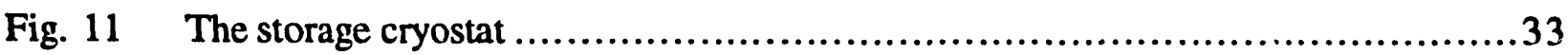

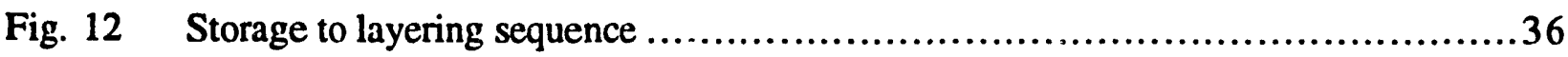

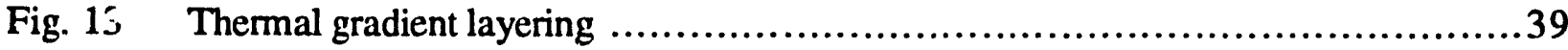

Fig. 14 Modified Mach-Zehnder Interferometer......................................42

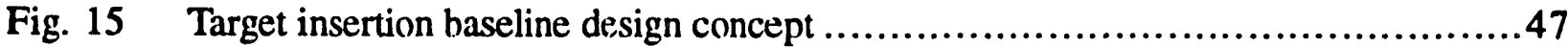

Fig. 16 Cross-section of cryogenic target insertion channel ..............................48

Fig. 17 Dual shroud system for protecting target.......................................49

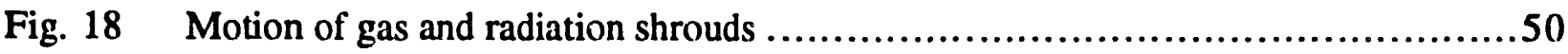

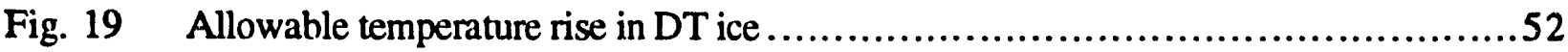

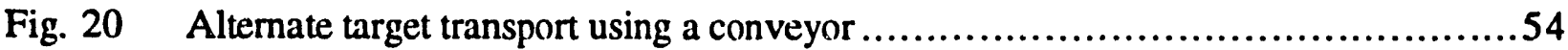

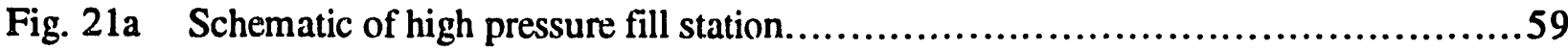

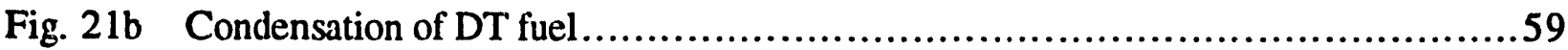

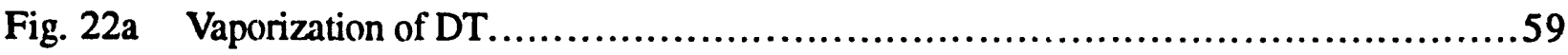

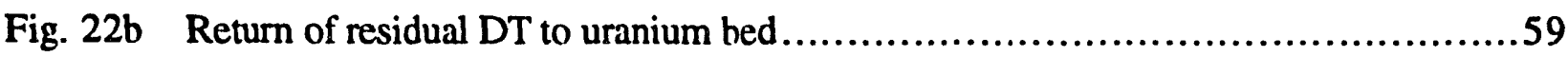

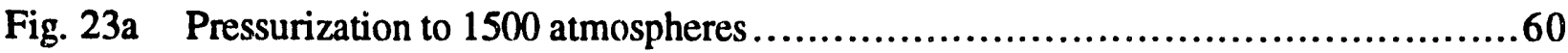

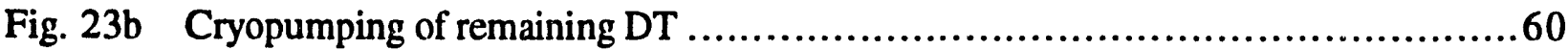

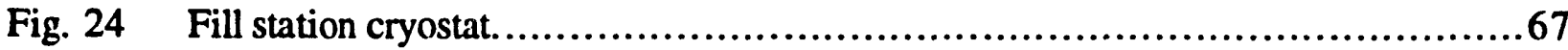

Fig. 25 Cryogenic extension wrench mated to the fill station cryostat.......................68

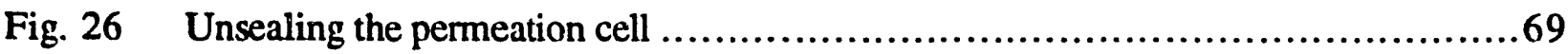

Fig. 27 Cryogenic extension wrench with the perneation cell cap.........................70

Fig. 28 Basic design of cold transport tube $\# 1$........................................... 71 
Fig. 29 Transport tube \#1 mated to the fill station cryostat.............................72

Fig. 30 Cryo-shroud being removed from transport tube $\# 1$............................73

Fig. 31 Transport tube \#1 picking up target rack........................................74

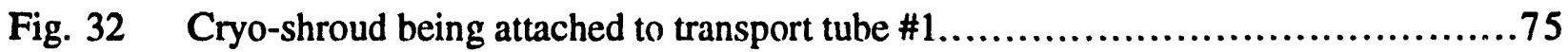

Fig. 33 Transport tube \#1 detached from the fill station cryostat...........................76

Fig. 34 Target rack and targets placed into the bottom of the storage cryostat ................77

Fig. 35 Layout of Target Delivery System ............................................8

Fig. 36 Transport tube \#2 attached to the top of the storage cryostat ......................85

Fig. 37 The basic design of transport tube \#2 ......................................86

Fig. 38 Transport tube \#2 picking up a target from the storage cryostat.....................87

Fig. 39 Transport tube \#2 detached from the storage cryostat .............................88

Fig. 40 Transport tube \#2 is attached to the top of the layering cryostat.....................89

Fig. 41 Upper horizontal section of layering cryostat.................................90

Fig. 42 Lower horizontal section of layering cryostat ...............................91

Fig. 43 Placement of interferometers, layering jets and transport arm.....................92

Fig. 44 Maximum radius for the target inset post ......................................93

Fig. 45 Position of $f 1.6$ lens in the interferometer system .................................94

\section{TABLES}

Table I Omega Upgrade tritium till requirements....................................... 16

Table II Summary of design requirements............................................57 


\section{INTRODUCTION}

The upgrade of the Omega laser at the University of Rochester's Laboratory for Laser Energetics (UR/LLE) will result in a need for large targets filled with $\mathrm{D}_{2}$ or DT and maintained at cryogenic temperatures. This mandates a cryogenic tazget delivery system capable of filling, layering, characterizing and delivering cryogenic targets trs the Omega Upgrade target chamber. The program goal is to design, construct, and test the enti.e target delivery system by June 1996 . When completed (including an operational demonstraion), the system will be shipped to Rochester for reassembly and commissioning in time for the Omega Upgrade cryogenic campaign, scheduled to start in 1998. General Atomics has been assigned the task of developing the conceptual design for the cryogenic target delivery system. Design and áabrication activities will be closely coordinated with the University of Rochester, Lawrence Livermore National Laboratory (LLNL) and Los Alamos National Laboratory (LANL), drawing upon their knowledge base in fuel layering and cryogenic characterization. The development of a target delivery system for Omega could also benefit experiments at Lawrence Livermore National Laboratory and the other ICF Laboratories in that the same technologies could be applied to NOVA, the National Ignition Facility or the future Laboratory Microfusion Facility. 


\section{PURPOSE OF DOCUMENT}

The purpose of this document is to describe the conceptual design for the Omega Upgrade target delivery system. The conceptual design comprises individual modules that perform unique tasks. The discussion of each module includes a listing of the system design requirements. Bracketed numbers (in boldface) preceding each requirement are those used in the May 1992 Design Requirements Report (Attachment A). A baseline design is listed along with optional methods that were considered for each task. The recommended approach is compared to the design requirements and problem areas (if any) noted. Research and development needs are identified, especially in areas where risk requires backup approaches.

We believe that a final design can be developed from this conceptual design that satisfies all the design requirements. However, it must be recognized that this design is preliminary. It is possible that certain requirements (existing or future) may prove to be incompatible with our implementation of the conceptual design. Evaluation of the conceptual design against the design requirements will provide a mechanism for identification and resolution of any such incompatibilities. The conceptual design process was also used to identify areas where the design requirements need to be modified (e.g., the requirement for opaque shells-not specified as a requirement in the May 1992 Design Requirements Report-and the relaxation of the thermal constraints on the transport of layered targets to $\pm 0.5 \mathrm{~K}$ ). The basis for the need to modify the Design Requirements Report will be discussed in the appropriate module. 


\section{DESIGN DESCRIPTION}

\subsection{SUMMARY DESCRIPTION}

The target delivery system can be thought of as modules: Target Support, Cryogenic Fill Station, Transport, Layering, Characterization and Target Insertion. Suitable plastic target shells will be supplied by GA. These targets will be mounted, filled with appropriate fuel gases, cryogenically cooled and transported into a module that will create a uniform solid fuel layer. They will then be characterized and transported into the target chamber. A cryogenic storage system will also be developed to allow for transport of filled targets and for inventory purposes. A flow diagram of the process is shown below.

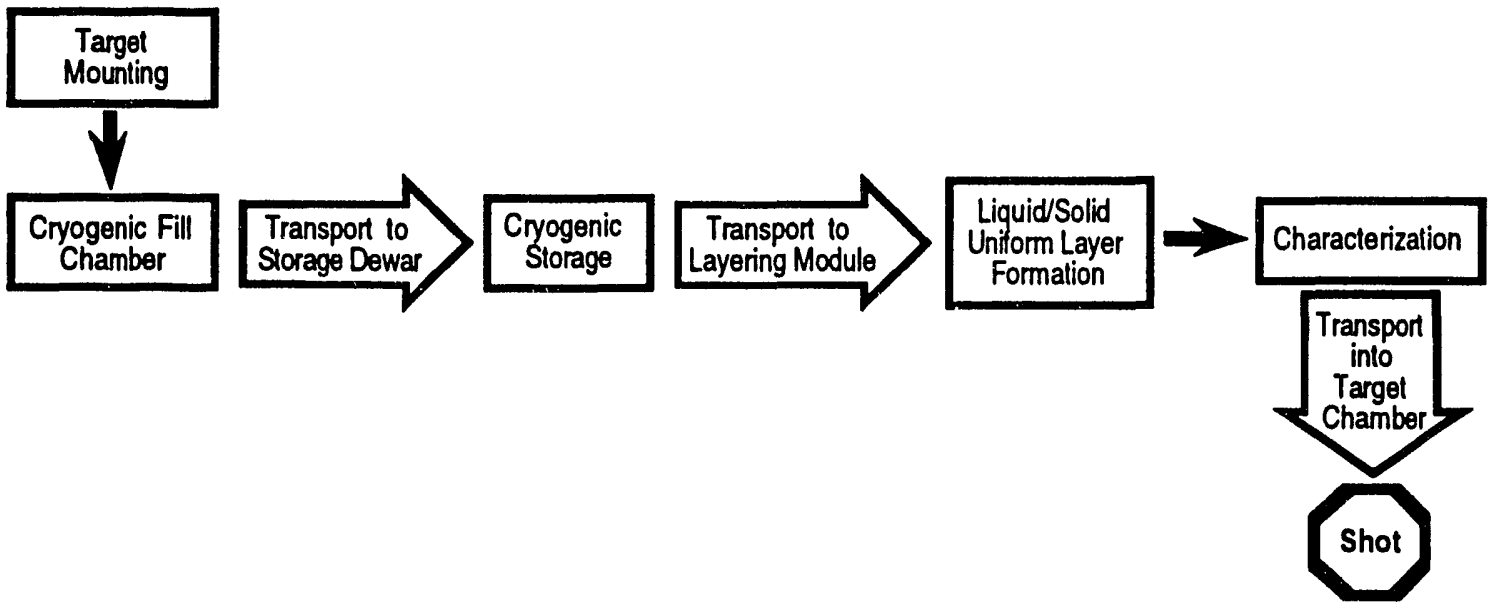

\subsection{PHYSICAL CONFIGURATION}

To better visualize the actual movement of a target, the physical layout of the target delivery system is described below:

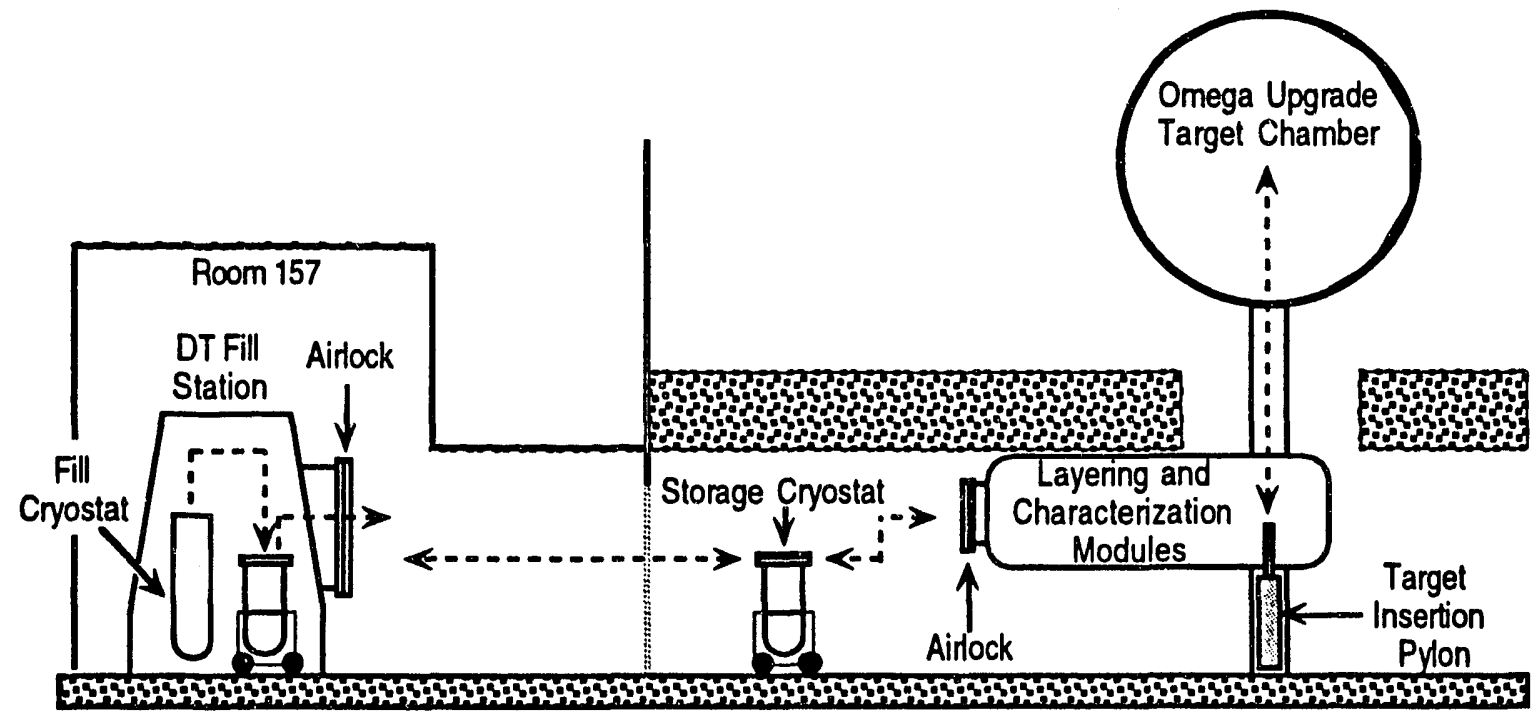

Figure 1. Schematic layout of Target Delivery System (not to scale). 
1) The empty targets are placed in a target rack within the Fill Cryostat, filled and then cooled below the critical point of the fill gas ( $D_{2}$ or DT).

2) The excess DT is pumped away. The permeation cell (in the Fill Cryostat) is then opened with a cryogenic wrench (not shown).

3) A Cryogenic Transport Mechanism (not shown) is used to transfer the targets from the Fill Cryostat to the Storage Dewar (still within the DT fill station glove box).

4) The Storage Cryostat is moved through the air lock of the fill station glove box and on to the room containing the Layering and Characterization Cryostat. This room is located in the basement directly below the Omega Upgrade target chamber.

5) After the Storage Cryostat is moved through an airlock into the Layering and Characterization Cryostat, another Cryogenic Transport Mechanism is used to transfer a single target from the Storage Cryostat into the Layering Module.

6) After the target has been layered and characterized, it is placed at the tip of the Target Insertion pylon.

7) The Target Insertion pylon is elevated and positions the target at the center of the Omega Upgrade target chamber.

8) Thermal radiation shrouds surrounding the target are retracted exposing the target to the laser optics. Within $\sim 30$ milliseconds after the shrouds are removed, the laser is fired.

\subsection{INTEGRATION WITH THE REST OF THE OMEGA UPGRADE PROGRAM}

Although not explicitly discussed in this conceptual design, each module's function will be designed to integrate with all other aspects of the Omega Inertial Confinement Fusion Program. Prior to the final design of the Omega Upgrade Target Delivery S:-stem, every aspect of the design will be examined to ensure compatibility with the rest of Omega Upgrade (e.g., interlocks to prevent laser misfire). Specific attention will he paid to interface requirements imposed by other systems in Omega Upgrade. All instrumentation and control functions will be designed to follow UR/LLE protocols. Specific care will be taken to ensure that all mechanical constraints on weight, cooling water, dimensions, etc. are known well before designs are finalized. Likewise, all electrical designs (line voltages, required power, duty cycle, etc.) will be matched to UR/LLE's requirements and capabilities. Finally, the strictest attention will be paid to environmental safety and health (ES\&H) considerations, especially those imposed by the requirement to operate with tritium. 


\subsection{TIMELINE}

\section{MODULAR APPROACH}

Omega is scheduled to shut down operations in December 1992. The Omega Upgrade is expected to begin non-cryogenic operations in 1995 . Our overall goal is to have the complete cryogenic target delivery system in place, fully tested, and performing to the design specifications when the rest of the Omega Upgrade is ready for a cryogenic campaign, currently scheduled for mid-1998. The baseline milestone schedule, Fig. 2, shows how we intend to have the system ready on time.

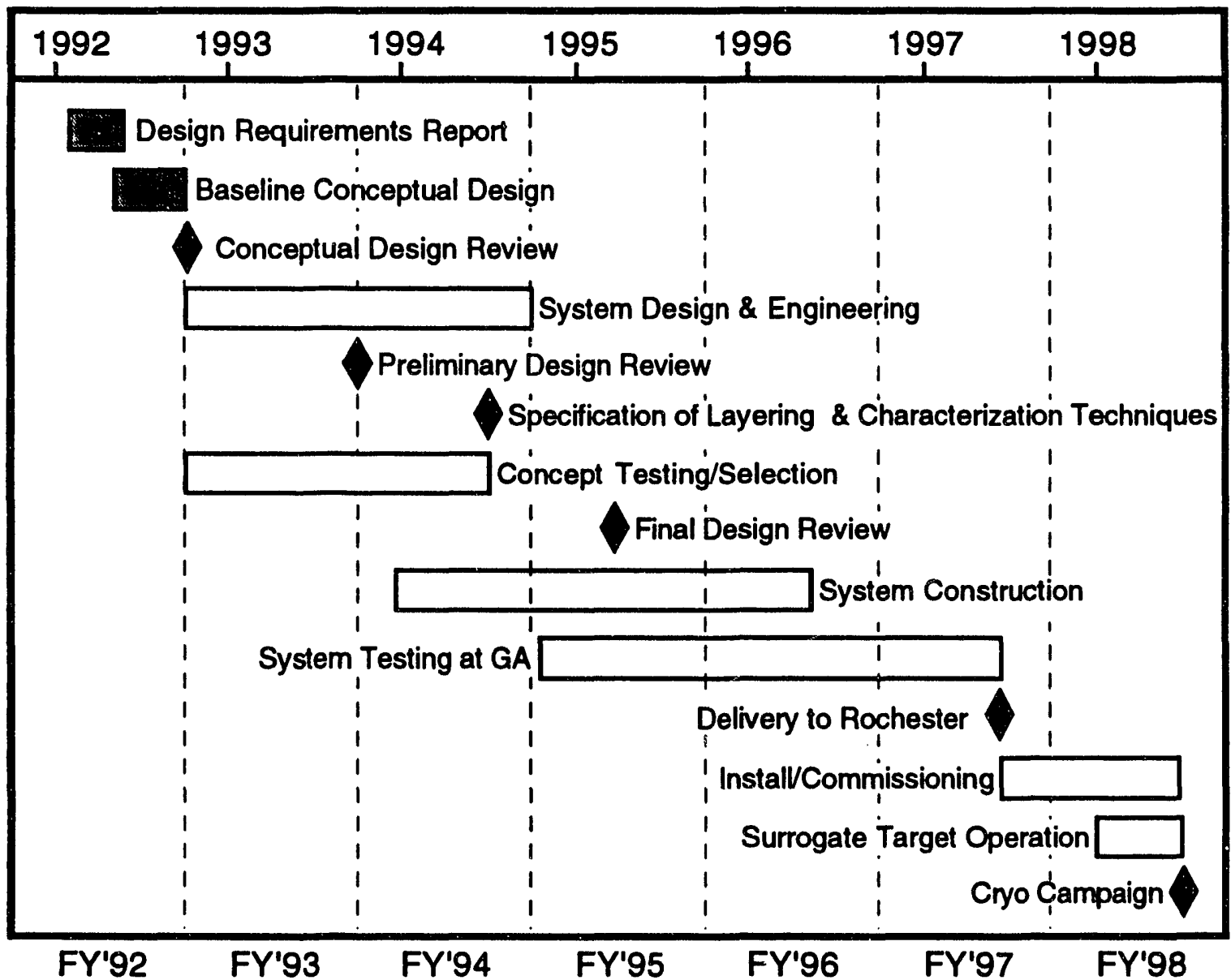

Figure 2. The milestone schedule shows how we expect to have a proven system ready for the Omega Upgrade cryogenic campaign. Appropriate funding and a timely demonstration of the layering and characterization techniques are critical to meeting the schedule.

The schedule is based on two major, yet uncertain, assumptions: a) funding on a year-to-year basis is appropriate for the work scheduled, and b) the techniques for the two items of greatest technical uncertainty, layering and characterization, are identified and demonstrated to be suitable for Omega Upgrade cryogenic targets by December 1993. The failure of either of the assumptions will substantially delay the schedule. 
Unfortunately the funding for FY93 is marginal. All reasonable candidate techniques ought to be seriously explored for layering and characterization to increase the chance that suitable techniques will be demonstrated by December 1993. However only two layering techniques (thermal gradient and beta-layering) and three characterization techniques (interferometry, ultrasonic, and NMR) will be funded. FY93 funding is based on the assumption that techniques from this list will prove to be acceptable.

In FY93, we will perform concept testing and selection. This entails assisting LLNL and LANL with exploring layering and characterization techniques, and determining appropriate materials of construction for the target support, fill station, transport, and cryogenic storage modules. In addition we will perform system design and engineering on the target support, fill station, transport, and cryogenic storage modules. A preliminary design review on those modules is scheduled for October 1993.

In FY94, we will assemble and test mockups of the modules, re-engineering or re-designing when appropriate. The final design review is scheduled for Octoher 1994. Since the layering and characterization techniques may not be identified until December 1993, the layering and characterization modules will need extraordinary efforts to be ready for the final design review. Substantial capital equipment money will be required in FY94. The staff will be approximately tripled.

In FY95, we will complete testing and re-engineering the modules. Final construction will begin on all modules except for the DT fill station. Funding for FY9s will be roughly equivalent to FY94.

In FY96, all modules will be fully constructed and tested (except for the DT fill station) to ensure that all components perform to design specifications and integrate with each other. In mid-year the system will be disassembled for shipment to LLE. Installation at LLE will begin. Construction of the DT fill station will begin.

In FY97, installation of the system at LLE will be completed. Working closely with LLE staff, we will test and improve the system as appropriate. Construction and final testing of the DT fill station will be completed. Licensing for tritium operations by the entire cryogenic target delivery system will be completed by LLE personnel.

In FY98, testing and commissioning will be completed in time for the start of the cryogenic target campaign.

In summary the milestone schedule is possible provided that funding and technical developments occur as required. We will perform more detailed planning and cost estimates after this conceptual design report has been accepted by UR/LLE and LLNL. 


\subsection{UNFILLED SHELL CHARACTERISTICS}

\subsubsection{Introduction}

Aithough shell production is not parı of this task, the shells used must satisfy specific criteria (diameter, sphericity, wall thickness and concentricity, surface finish, etc.). The shell material will be a $\mathrm{CH}$-based polymer plastic. The need to reduce the atomic number in the shell material precludes the use of glass shells. Shells will be procured from normal target fabrication production runs. It may be necessary to modify these production shells to meet the reqrirements of a cryogenic target.

\subsubsection{Design Requirements}

[4.2.1] The target delivery system will utilize 700-1100 $\mu$ m diameter $\mathrm{CH}$-based polymer shells filled with $\mathrm{D}_{2}$ or DT.

[4.2.2] The shell wall thickness shall be $5-10 \mu \mathrm{m}$.

\subsubsection{Options Considered}

Glass $\left(\mathrm{SiO}_{2}\right)$ is an alternate material, but has the disadvantage of higher atomic number and density compared to plastic.

\subsubsection{Baseline Design Description}

\section{Shell Material}

The shell material will be a $\mathrm{CH}$-based polymer. The physical properties (e.g., yield strength, permeability, radiation damage) and dimensions of the polymer(s) used will be important in determining DT and $\mathrm{D}_{2}$ permeation rates and fill pressures. Permeation data ${ }^{23}$ indicate reasonable rates for candidate materials such as polystyrene.

\section{Physical Quantities}

The shell dimensions will be determined by experimental requirements. On the basis of current estimates ${ }^{4}$ of Omega Upgrade targets, the shell diameter will range between 700 and $1400 \mu \mathrm{m}$, with wall thicknesses from 5 to $25 \mu \mathrm{m}$. These values more than exceed design requirements [4.2.1 and 4.2.2]. The shape shall be spherical with less than $2 \%$ departure from sphericity. Wall concentricity deviations [(outer diameter - inner diameter)+ mean diameter] must be below 2-3\%. The surface finish of selected shells must be at the

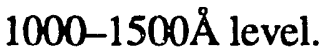

\footnotetext{
'Bracketed numbers (in boldface) are those used in the May 1992 Design Requirements Report.

${ }^{2}$ L. A. Scott, R. G. Schneggenburger and P. R. Anderson, "Deuterium permeation properties of beta-irradiated and unirradiated poly (vinyl alcohol) and polystyrene shells", J. Vac. Sci. Technol. A 51155 (1986)

3 G. W. Collins, J. J. Sanchez and E. M. Fearon, "DT-Filled, Plastic Direct-Drive Targets", LLNL ICF Quarterly Report 1141 (1991)

4 per H. Kim and S. Letzring. UR/LLE
} 


\section{Overcoating(s)}

The permeability of plastic decreases exponentially with decreasing temperature. Thus no overcoatings for the purposes of creating a permeation barrier will be required. Overcoatings may be needed to increase wall thickness to $25 \mu \mathrm{m}$. Although not called out as a design requirement, specific targets may require shine-through barriers or ablator coatings. The effect of shine-through barriers or ablator material on opacity (affecting characterization methods) and permeation rates will have to be assessed for each material.

\subsubsection{Comparison to Design Requirements}

We expect that R\&D for microencapsulation techniques in FY93 will lead to a tinnely supply of target-quality plastic shells with diameters exceeding $1,400 \mu \mathrm{m}$ and wall thicknesses in the 5-19 $\mu \mathrm{m}$ range (requirement [4.2.1 and 4.2.2]). Overcoating with materials such as $\mathrm{CH}$ (via GDP coating or parylene) should satisfy the need for wall thicknesses above 20 $\mu \mathrm{m}$.

The need for finished targets to have an optical shine-through barrier for $351 \mathrm{~nm}$ light was not included in the original design requirements. If this is truly a target requirement as determined by UR/LLE, the design requirements report will have to be amended. Shinethrough barriers are further discussed in the characterization section.

\subsubsection{Problem Areas, $R \& D$ Requirements and R\&D plans}

It is assumed that standard shell production methods will yield product of the appropriate diameter, concentricity, sphericity, surface finish, etc. It may be necessary to develop overcoating techniques if siandard shell production techniques (e.g., microencapsulation) are not sufficient to produce thick wall shells when required. It should be noted that this is not a target delivery system task, but is mentioned to identify a potential problem.

\subsubsection{Backup Approaches}

Opaque targets have been produced utilizing 1000-1500 $\AA$ aluminum coatings. Unfortunately, this yields a barrier that is nearly impermeable to hydrogen. An absolute need for an opaque, hydrogen impermeable shell would require a completely different approach to filling of DT and $D_{2}$ targets. Foam targets are a potential backup, both as a shell and a layering method.

\subsubsection{Summary}

With the exception of the shine-through barrier requirement, acquisition of target quality shells is considered a low risk item. The inability to develop a $351 \mathrm{~nm}$ opaque, but visibly transparent shell material or coating will significantly increase the need for alternative characterization methods. 


\subsection{TARGET SUPPORT}

\subsubsection{Introduction}

The purpose of the target support is to place the filled target at the focus of the Omega Upgrade laser beams and to maintain it during the laser shot. Obviously, the target will need to be rigidly held and the mounting method must not interfere with the laser beams or associated diagnostics in Omega Upgrade. Additionally, the target mounting method must not cause a major perturbation on surface finish, as this could instigate Rayleigh-Taylor instabilities.

Current Omega targets $(\sim+\mathrm{mm}$ diameter) are supported between spider webs, and glued in place with a thin coating of parylene. The proposed cryogenic targets will be about $4 x$ larger, and contain 50-100x as much fuel as present targets (up to $0.15 \mathrm{mg}$ DT). Omega Upgrade targets will be filled with $D_{2}$ or DT at pressures in excess of 1,000 atmospheres (at room temperature). Thin plastic shells can only survive relatively small differential pressures. To prevent bursting the shell, the target must be refrigerated to cryogenic temperatures, reducing the internal pressure to a few atmospheres (or less). This allows the filled target to be removed from the permeation cell without rupturing when the fill gas is removed.

Because of the difficulty in handling bare, filled targets at cryogenic temperatures (especially DT-filled), it is desirable to have some sort of holder to reduce (or eliminate) thermal and mechanical shocks to the target during transport and insertion operations. Preferably, a single holder performs this function as well as providing a support for the target during the actual laser shot.

\subsubsection{Design Requirements}

[4.3.1] The target mounting method must he thermally and mechanically stable to hetter than $\pm 5 \mu \mathrm{m}$ dimensionality.

[4.3.2] The target mount must not interfere with any of the 60 Omega Upgrade laser beams or associated diagnostics.

Although not a formal requirement, the target mounting method must not cause a major perturbation on (the target) surface finish.

\subsubsection{Options Considered}

A number of options for target support were considered (crescent mounting, pendulum and pedestal mounts, free-fall target injection, etc.). Compared to crescent mounting (the current method for mounting targets for Omega), a single stalk or pendulum mount minimizes contact to the target. This also requires a glue that will survive a single thermal cycle while 
loaded in tension. To date, no glue has been found that will survive thermal cycling and retain the strength to hold a target without using excessive amounts of glue. Stalk or pendulum mounts also exhibit significant lateral (pendulum) displacements. In the (vacuum) environment of the Omega Upgrade chamber, no damping forces exist to reduce lateral displacements that could easily exceed the $\pm 5 \mu \mathrm{m}$ positioning constraints for targets.

A modification of the existing UR/LLE copper crescent/spider web was seriously considered. This method is "proven", but requires a significantly larger dead volume for the DT fill station. This severely impacts the ability of the DT fill station to meet UR/LLE's tritium site license. Additionally, the crescent mount requires a convoluted non-planar shape to avoid Omega Upgrade's laser beams.

\subsubsection{Baseline Design Description}

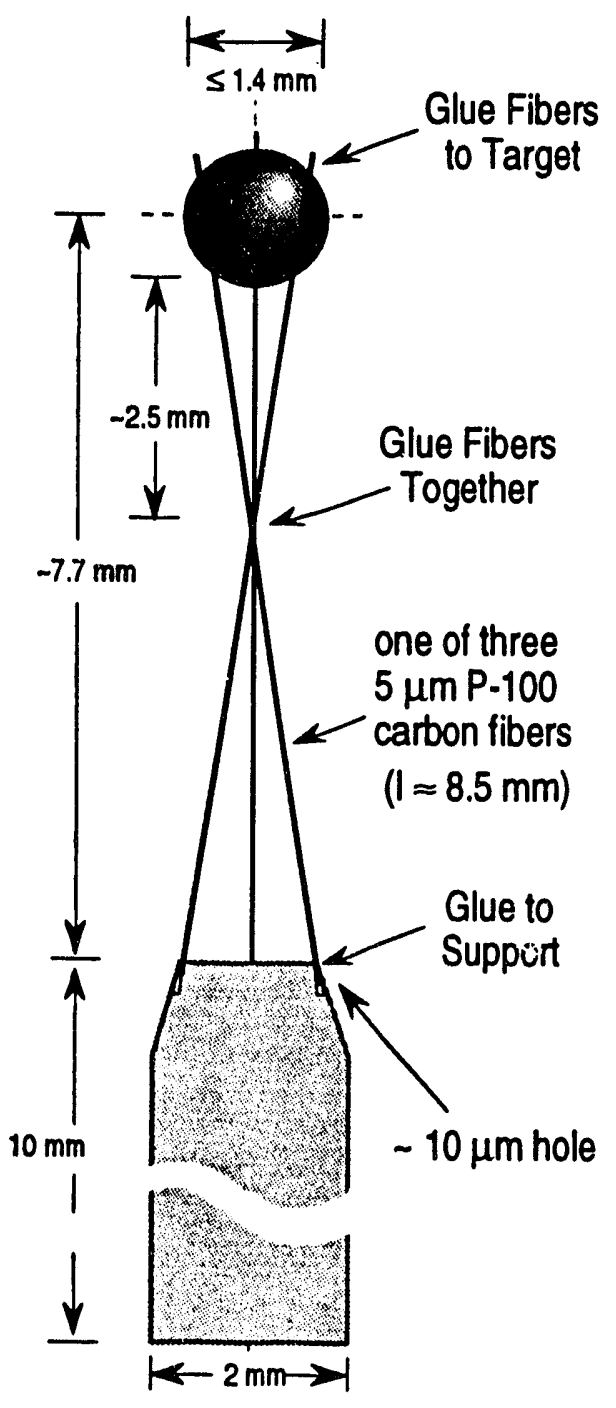

Figure 3 Tripod Target Mount
A tripod mounting technique was chosen as the preferred design. The target support is a stiff, three-legged carbon fiber support that is glued in several locations (Fig. 3). Unlike a hanging pendulum mount where the glue is loaded in tension, the glue attaching the three carbon fibers is loaded in compression or shear. The glue causes the target to be part of the support structure, furthe: increasing its strength. Commercially available carbon fibers can be quite thin $(<5$ $\mu \mathrm{m}$ diameter) and long (>10 mm). The use of long length, small diameter carbon fibers minimize both thermal conduction and contact area to the target. Positioning the fiber intersection at least $3.2 \mathrm{~mm}$ below the center of the target and using a $2 \mathrm{~mm}$ diameter support cylinder ensures minimal interference with the (60) Omega Upgrade laser beams (Fig. 4 ).

Unlike a crescent mount, where the prongs of the mount must be $1 \sim 2 \mathrm{~cm}$ apart, the height and diameter of the tripod mount minimize the DT dead volume required in the DT fill station permeation cell $(<0.1 \mathrm{cc})$. Additionally, the tripod mount design allows for bottom positioning of the Target Insertion system in the Omega Upgrade target chamber, a significant design advantage over suspended mounting techniques. The $-2 \mathrm{~mm}$ o.d. of the support cylinder allows for a smaller diameter radiation shroud in the Target Insertion module. This decreases shroud mass, yielding higher accelerations and shorter shroud retraction times 


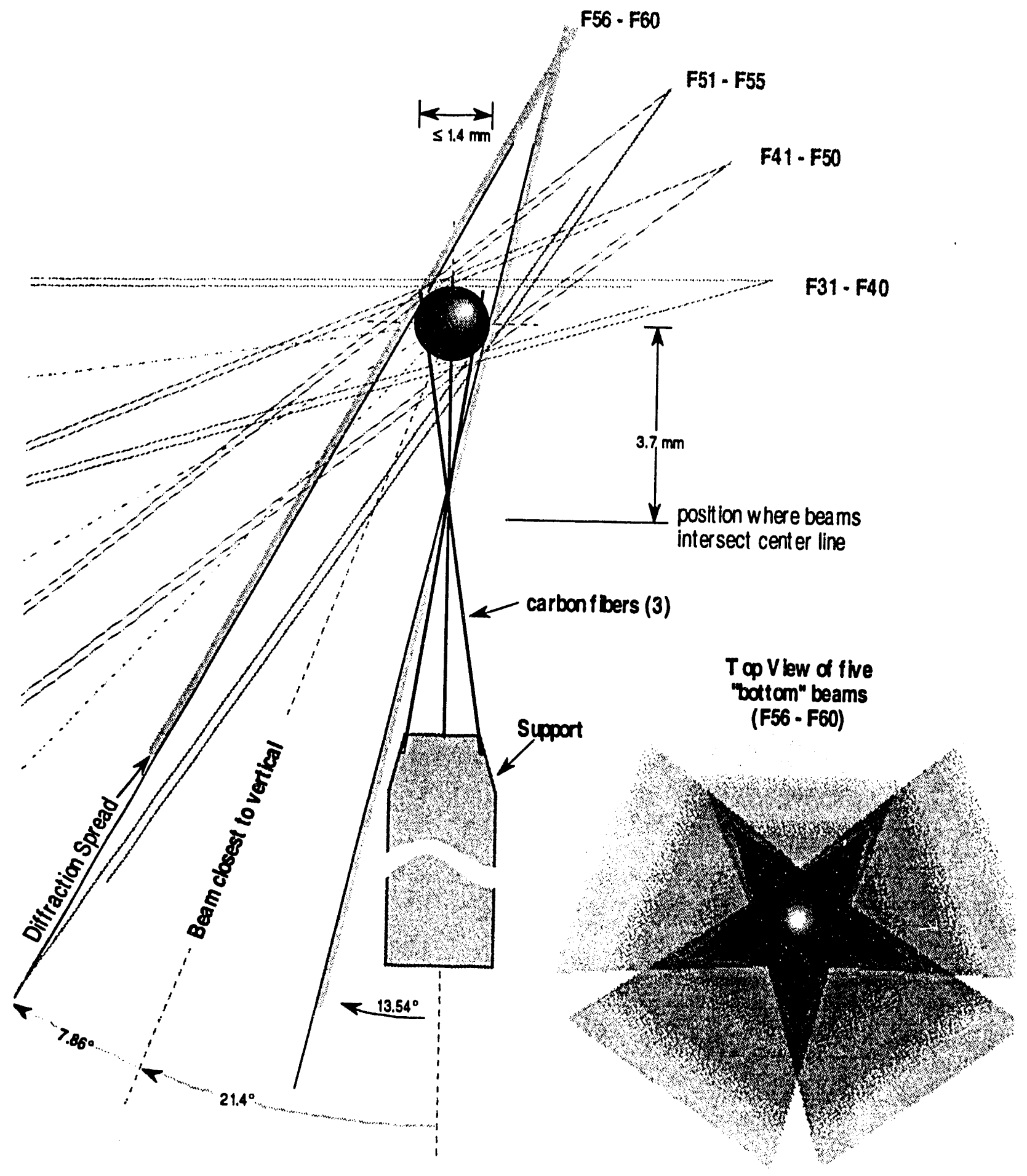

Figure 4 Beam Paths for Omega Upgrade 
The inherent rigidity of a tripod allows accurate positioning of the target. Both vapor grown and P-100 carbon fiber have adequate modulus ( $>600 \mathrm{GPa})$ and tensile strength $(2-7$ GPa) to support large DT-filled targets appropriate to Omega Upgrade. Even without gluing the tripod intersection, the buckling stability is sufficient to support a filled target.

A finite element analysis was used to examine the tripod mount concept. The finite element COSMOS/M computer code was used to determine stresses, deflections and resonant frequencies. The resonant frequencies found were:

$22.4 \mathrm{~Hz}$ for lateral motion

$77.2 \mathrm{~Hz}$ for torsional motion

$32.9 \mathrm{kHz}$ for "whiplash" $(X-Y)$ motion

$33.6 \mathrm{kHz}$ for vertical motion

The conclusion of the finite element analysis is that the tripod mounting technique is stable to buckling with a safety factor of three. This factor can be increased four-fold by extending the base conically to the fiber intersection and gluing the fibers to the cone. The greatest deflection is in torsional motion, but even this is survivable-a moment equal to the target weight $x$ target radius gives only a $12^{\circ}$ target rotation. Finally, the vertical deflection from the target weight is $0.001 \mu \mathrm{m}$. The conclusion of the finite element analysis is that the tripod mounting technique will survive reasonable handling.

\subsubsection{Comparison to Design Requirements}

We believe that the proposed design concept meets all design requirements. The contact dimensions $(<5 \mu \mathrm{m})$ minimize surface perturbations. The high modulus and tensile strength of the carbon fibers maximize support rigidity (e.g., predicted vertical deflections three orders of magnitude below that permitted by the design requirements [4.3.1]). Finally, the tripod design minimizes interference with the Omega Upgrade laser beams ([4.3.2]) .

\subsubsection{Problem Areas, $R \& D$ Requirements and $R \& D$ plans}

The tripod mounting technique has not yet been built. Dr. Hyo-gun Kim at the University of Rochester has agreed to perform the research and development to establish the preferred target support design. We believe that this can be performed over the next six months. Failure of this concept requires significant redesign of the DT fill station module. One concern to be addressed is if existing glues, held in shear and/or compression, will survive thermal cycling (It should be recognized that actual targets will only be subjected to a single thermal cycle. Once a target is filled and cooled, it will always be kept at cryogenic temperatures.) The support cylinder material needs to be decided upon as well as an adequate stalk length to avoid debris damaging the insertion device. We do not believe that the carbon fiber properties will not degrade with radiation dose (beta decay). We need to 
determine if the target support or carbon fiber adsorbs tritium during the DT-fill and is later given off. Finally, it may be of interest to consider five fiber configurations to symmetrize the shadows (from the five-fold symmetry of the 60 Omega Upgrade laser beams) or configurations where the fibers do not intersect.

\subsubsection{Backup Approaches}

Should Dr. Kim's research show the tripod mounting technique to be unrealistic, a modification of the existing UR/LLE copper crescent/spider web will be used

As noted, the significantly larger dead volume of a crescent mount impacts design of the DT fill station permeation cell. In the existing crescent mount, a thin parylene coating is used to glue the shell to the spider webs. We will need to verify that this is appropriate for the larger Omega Upgrade targets. Additional strands of spider web may be necessary to support a $>1$ $\mathrm{mm}$ filled shell. And, like the carbon fiber tripod mount, we need to verify that the spider web properties will not degrade with radiation dose(s) or if the target support and/or spider web adsorb tritium.

\subsubsection{Summary}

As mentioned earlier, the tripod mounting technique meets all design requirements. It is a low risk item whose proof-of-principle can be verified in a relatively short period. It uses commercially available existing carbon fibers whose mechanical properties exceed those needed for a rigid mount. It offers minimal dead volume and allows bottom positioning of the target insertion system. Finally, a viable, but less desired, backup method (the crescent mount) exists.

\subsection{CRYOGENIC FILL STATION}

\subsubsection{Introduction}

The need for thick $(100 \mu \mathrm{m})$ liquid or solid layers of $D_{2}$ or DT fuel in large diameter plastic shells requires that empty targets be filled to densities as high as $0.16 \mathrm{gm} / \mathrm{cc}$, equivalent to 1500 atmospheres at room temperature. During fills to high pressure, polymer targets have to be filled in steps of less than 10 atmospheres to keep the targets from collapsing. High pressure fills in polymer capsules require maintaining shells at cryogenic temperatures after filling to prevent the shells from bursting. This, combined with the requirements that the gas permeate into the shells, implies that the system must work in a temperature range from room temperature down to as low as liquid helium temperatures. It is assumed that no targets will require overcoatings that are significantly less permeable to $D_{2}$ or DT than that of the $\mathrm{CH}$-based polymer used to make the shells. Factors that affect the design of the fill station include requirements for target throughput and tritium inventory capacity. The fill 
station must be designed to the highest environmental safety and health standards. Emphasis will be on design of the DT system. It should be noted that the DT fuel is really a mixture of isotopic $D_{2}$, DT and $T_{2}$ in a 1:2:1 ratio. Except for radiological safety constraints, the $D_{2}$ system requirements are identical. Both $\mathrm{D}_{2}$ and $\mathrm{DT}$ fill stations will be identical, except that the $D_{2}$ fill station will not be located in a triple containment environment, and the $D_{2}$ will be supplied from a high pressure gas bottle rather than a uranium DT bed. The $\mathrm{D}_{2}$ fill station will be constructed first and serve as a prototype for the DT fill station.

Even though the surrogate cryogenic target campaign will not start until 1998, there will be a requirement for low pressure $\mathrm{D}_{2}$ and DT fills when Omega Upgrade comes online in 1995. The UR/LLE tritium fill requirements up to and including the year 2000 are summarized in Table I.

\begin{tabular}{|c|c|c|c|c|c|c|}
\hline \multirow{3}{*}{ Year } & \multicolumn{5}{|c|}{ Table I } & \multirow{3}{*}{$\begin{array}{l}\text { \# targets } \\
\text { per year }\end{array}$} \\
\hline & \multirow[t]{2}{*}{ Typet } & \multicolumn{2}{|c|}{$\begin{array}{l}\text { wall thickness } \ddagger \\
\mu \mathrm{m}\end{array}$} & \multicolumn{2}{|c|}{$\begin{array}{l}\text { average fill } \\
\text { pressure } \\
\text { atm. }\end{array}$} & \\
\hline & & glass & plastic & glass & plastic & \\
\hline FY95 & $\mathrm{SiO}_{2}+\mathrm{CH}$ & $1-50$ & $10-50$ & 5 & 30 & 400 \\
\hline FY96 & $\mathrm{SiO}_{2}+\mathrm{CH}$ & $1-50$ & $10-50$ & $\mathbf{5}$ & 30 & 800 \\
\hline FY97 & $\mathrm{SiO}_{2}+\mathrm{CH}$ & $1-50$ & $10-50$ & 50 & 30 & 800 \\
\hline \multirow[t]{2}{*}{ FY98 } & $\mathrm{SiO}_{2}+\mathrm{CH}$ & $1-50$ & $10-50$ & $\mathbf{5}$ & 30 & 400 \\
\hline & surrogate cryo and CDT polymer & & $10-50$ & & 30 & 400 \\
\hline \multirow[t]{3}{*}{ FY99 } & $\mathrm{SiO}_{2}+\mathrm{CH}$ & $1-50$ & $10-50$ & 5 & 30 & 200 \\
\hline & surrogate cryo and CDT polymer & & $10-50$ & & 30 & 100 \\
\hline & cryogenic $\mathrm{CH}$ & & $10-50$ & & $\leq 1300$ & $<100$ \\
\hline \multirow[t]{3}{*}{ FYOO } & $\mathrm{SiO}_{2}+\mathrm{CH}$ & $1-50$ & $10-50$ & $\mathbf{5 0}$ & 30 & 200 \\
\hline & surrogate cryo and CDT polymer & & $10-50$ & & 30 & 100 \\
\hline & cryogenic $\mathrm{CH}$ & & $10-50$ & & $\leq 1300$ & 100 \\
\hline
\end{tabular}

- Supplied by John Soures, UR/LLE ( $\mathrm{SiO}_{2}$ targets will be used for non-cryogenic experiments)

t Target diarneters range from 700 to $1000 \mu \mathrm{m}$

$\ddagger$ Glass target fill temperature $=360^{\circ} \mathrm{C}, \mathrm{CH}$ target fill temperatures $<77^{\circ} \mathrm{C}$, typically $25^{\circ} \mathrm{C}$

\subsubsection{Design Requirements}

[4.4.1] The target delivery system shall include the ability to fill empty targets with $D_{2}$ or DT gas to pressures greater than 1000 atmospheres at room temperature.

[4.4.2] The target delivery system shall include the ability to cool filled targets to below the triple point of the filled gas without rupturing the filled target.

[4.4.3] The tritium inventory of the DT fill station shall be less than one gram of tritium, but sufficient to fill at least six months supply of targets.

[4.4.4] The DT fill station must not release tritium to the environment. 
[4.4.5] The DT fill station must contain a backup system for tritium recovery.

[4.4.6] The DT fill station must include power failure protection.

\subsubsection{Options Considered}

While the option of building a completely new system was considered, our conceptual design study for the cryogenic fill station indicates that many of the basic design features of the existing UR/LLE tritiurn fill system seem to be acceptable to the needs of a future tritium fill facility. Components in the process system will need to be re-sized to correspond with the quantities of $D_{2}$, DT and $T_{2}$ required by the new target filling parameters. Safety features of the fill system will likely be added to and/or enhanced to assure safe operation with larger target and higher density fills. The maximum pressure for filling polymer shells will be 1500 atmospheres $(152,000 \mathrm{kPa})$ at $300 \mathrm{~K}$.

\subsubsection{Baseline Design Description}

\section{Processing Component}

The processing component of the cryogenic fill station will consist of the same features as those in the existing UR/LLE tritium fill system.

1) receiving and inventory of tritium and deuterium gases

2) pumping system for evacuation of process gases,

3) storage of tritium in stable hydride form,

4) target charging loop.

\section{Pumping System, Receiving and Inventory Loop}

The high vacuum and rougking pumps used in the existing UR/LLE tritium fill system seem satisfactory for the cryogenic fill system. No major change is anticipated for the receiving and inventory loop.

\section{DT Storage Loop}

The uranium beds, which store the DT at room temperature in the form of uranium hydride in the UR/LLE tritium fill system, each have a capacity of $9,600 \mathrm{Ci}(\sim 1 \mathrm{gram})$ of tritium with equimolar deuterium. Upon heating the beds to $450^{\circ} \mathrm{C}(723 \mathrm{~K})$, the DT is released/ desorbed from the uranium. The present UR/LLE tritium fill system has the capability to charge eight microballoons at $4.2 \mathrm{Ci}$, each using a working inventory of $8,000 \mathrm{Ci}(0.83$ grams of tritium). Careful design should be able to produce much higher density targets without an increase in working inventory. If this is the case, then similar size uranium beds to the UR/LLE tritium fill station can be used in the cryogenic fill system. Our preliminary design calculations show the working inventory of the fill system with 1,500 atmosphere 
capability can be limited to 0.79 grams of tritium ( 1 gram tritium $\equiv 1.67$ gram DT).

Since the uranium beds are the storage location of tritium, it has been suggested that additional safety be considered in the uranium bed design. There is already inherent safety built into the uraniuin beds since high heat of $650 \mathrm{~K}\left(373{ }^{\circ} \mathrm{C}\right)$ is required to drive hydrides off to recombine as $D_{2}, D T$ and $T_{2}$ in a 1:2:1 ratio. $A$ back-up over-temperature control will be used to prevent over heating of the uranium bed. But hydrides could be released if the temperature is inadvertently raised or if contamination occurs through leakage into the uranium bed causing oxidation. If the uranium bed enclosure cannot contain the tritium, then a secondary containment around the uranium beds will need to be added. E.g., a "jacket" enclosure which would allow circulation of this small enclosed volume around the uranium bed and through a detritiation system.

\section{Permeation Loop}

The permeation loop of the cryogenic fill system will require major design changes compared to the current UR/LLE tritium fill system (Fig. 5).

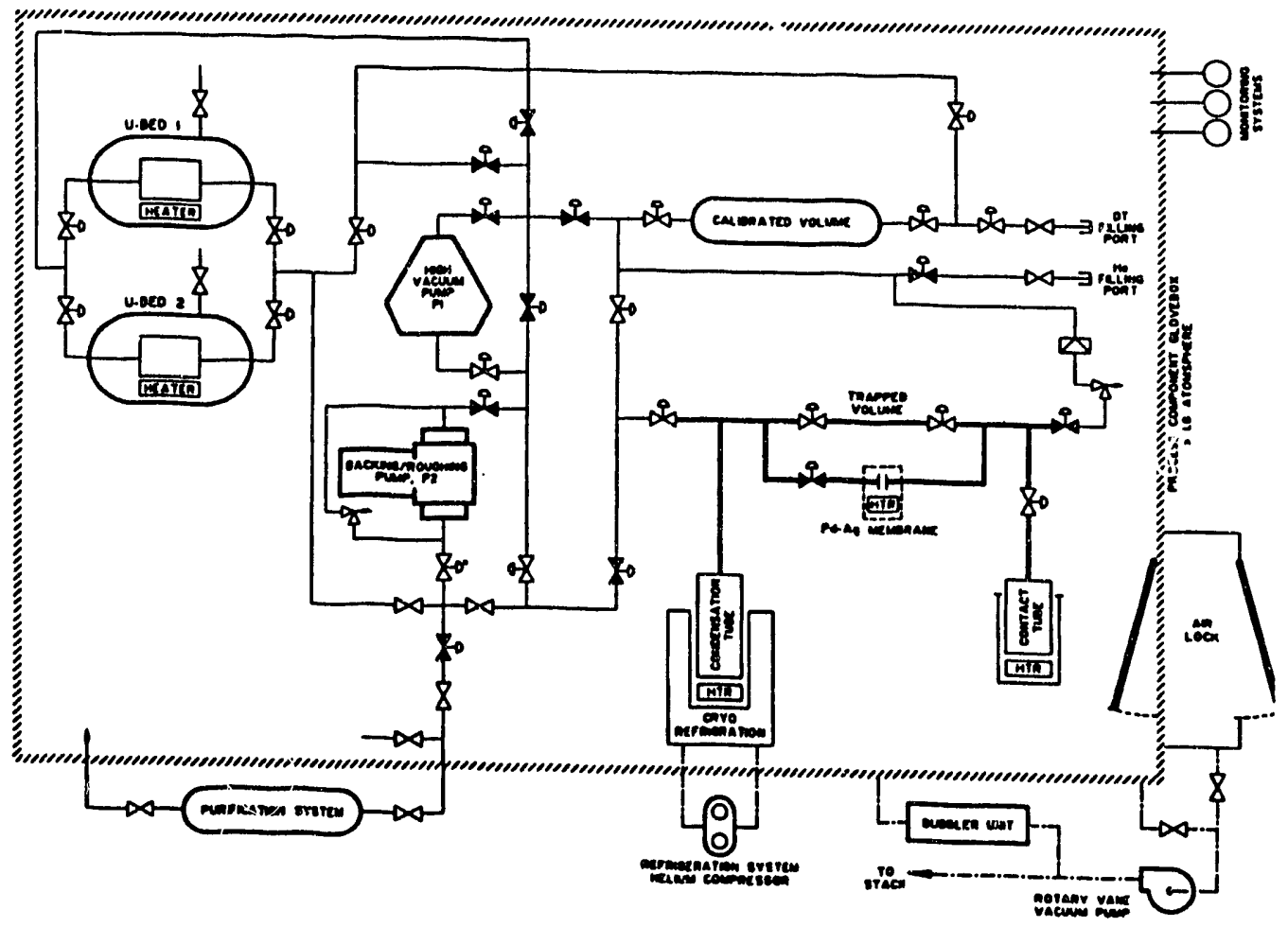

Figure 5. Simplified block diagram of the existing UR/LE tritium fill system.

The high pressure needed in the cryogenic fill system to produce high density targets will necessitate the use of higher rated equipment. 
The broad range of target fills will be difficult to achieve with a single permeation loop. The volume sizing of a permeation loop to accommodate a particular fill parameter will not lend itself to a wide variety of target densities. In addition, pressure measurement of a high pressure system will not have the degree of high accuracy needed for a low density target fill. Other permeation loop components such as valves and tubing may be selected for a high density target fill (high pressure), but may be impractical for a low density fill. Therefore, the cryogenic fill system will likely have more than one permeation loop, possibly two or three, to accomplish a wide range of target density fills. It is assumed that one of the loops would be that currently used in the UR/LLE tritium fill system.

The cryogenic fill system (Fig. 6) has only the one high pressure permeation loop shown (within the secondary containment), but this is only for clarification and the existing permeation loop would remain in the system parallel to the single permeation loop shown. Other components of the process system (i.e., receiving and inventory, pumping and DT storage) would be common to all permeation loops in the cryogenic fill system. Figure 7 shows the current UR/LLE fill station looking at it from the end, which will be modified to accommodate the high pressure fill station.

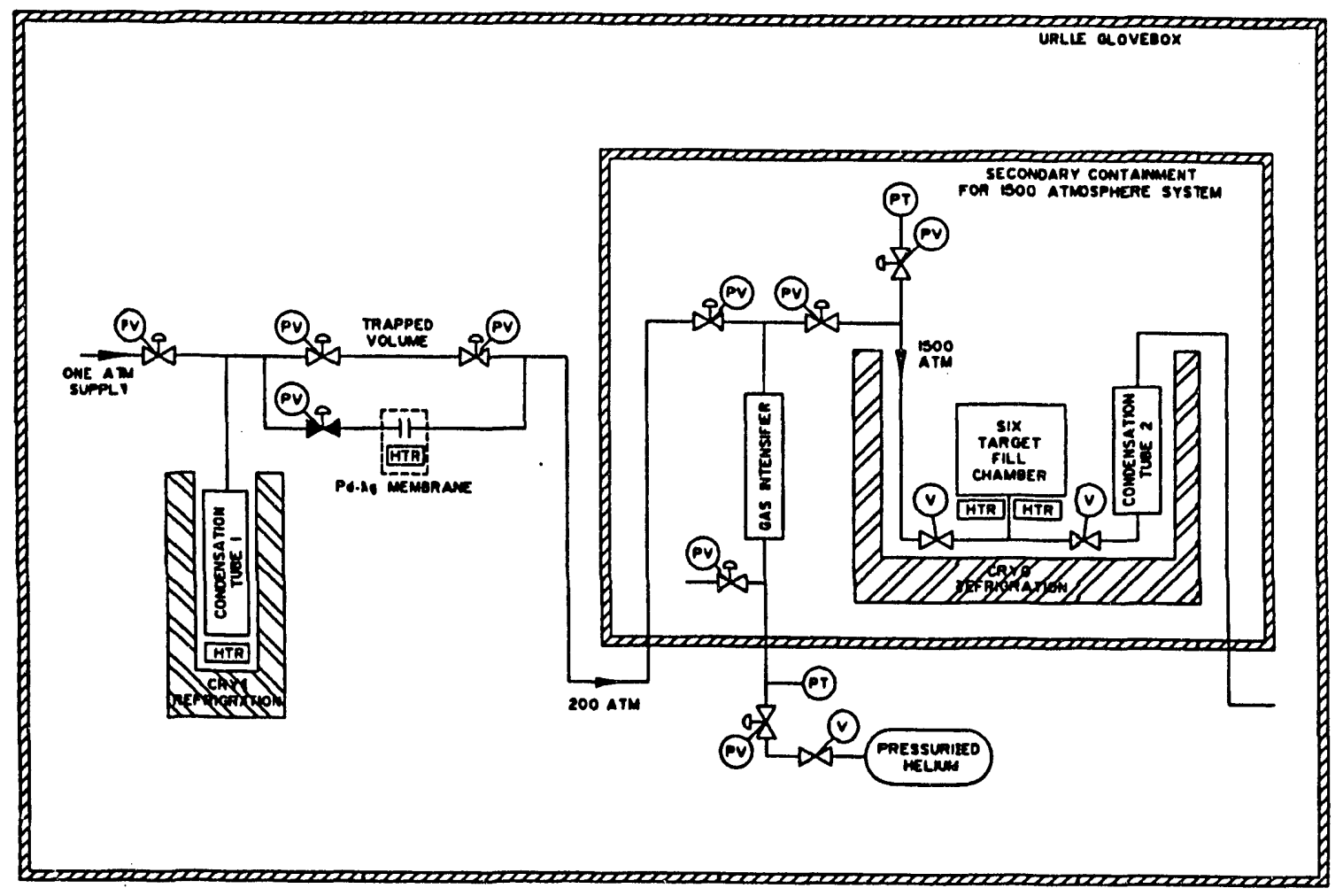

Figure 6. Block diagram of the proposed high pressure portion of the cryogenic fill system for performing high density target fills. 


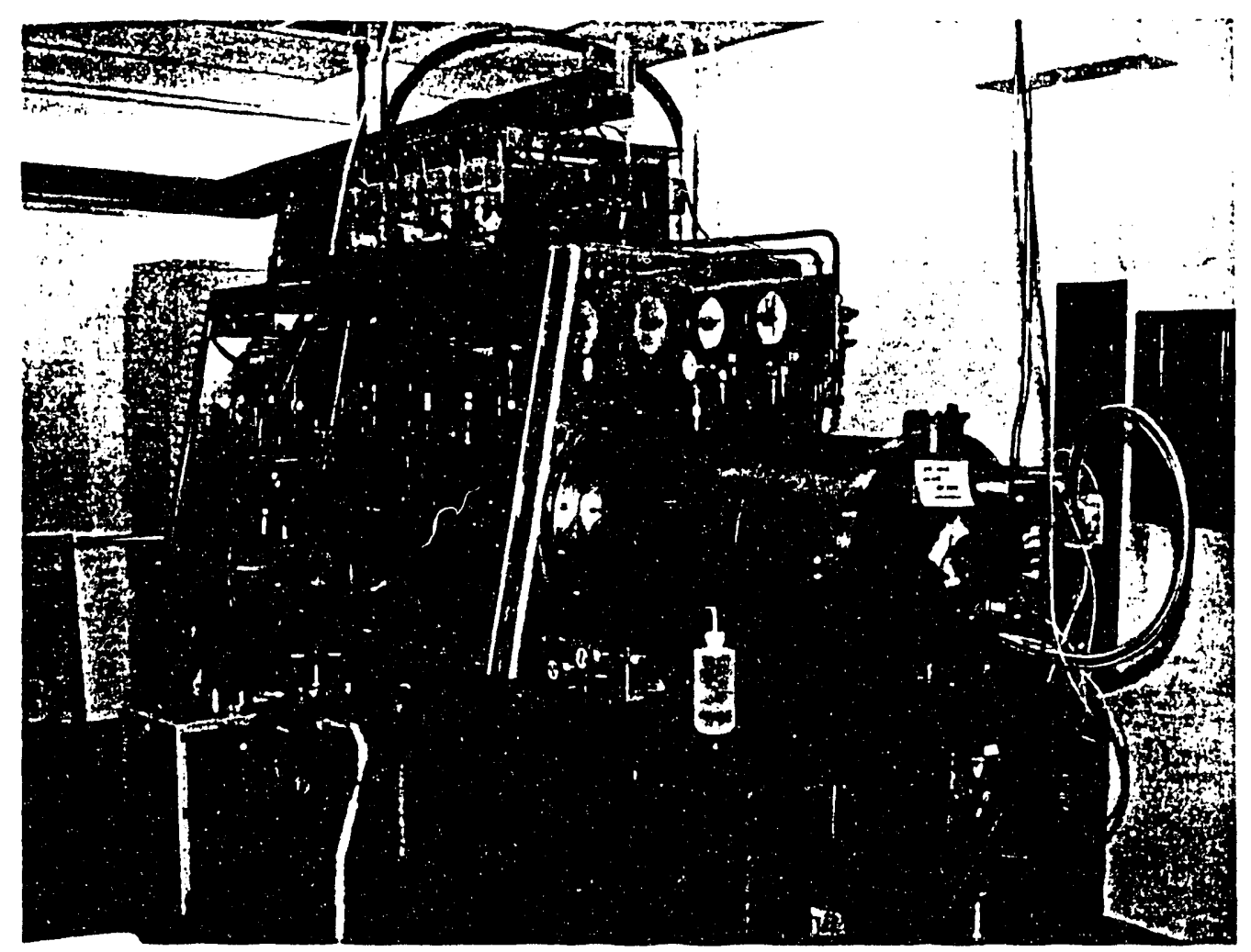

Figure 7. The existing UR/LLE DT fill station. A new section will be added between the glove box and the airlock to accommodate the cryogenic fill components.

Various methods can be used to achieve high pressures for a high density fill. The permeation loop volume can be decreased, or other pumping systems can be utilized. It appears that the current $9600 \mathrm{Ci}$ inventory used in the UR/LLE tritium fill system can produce the pressures (in a closed loop) required of a high density fill. The UR/LLE tritium fill system produces an equilibrium pressure of $15,600 \mathrm{kPa}(2260 \mathrm{psi})$ from an initial pressure of $23,80() \mathrm{kPa}(3450 \mathrm{psi})$ in the condensation loop. In the existing UR/LLE fill system, the volumes of the condensation and permeation loops are $37 \mathrm{cc}\left(2.25 \mathrm{in}^{3}\right)$ and 17.4 cc $\left(1.06\right.$ in $\left.^{3}\right)$ respectively. Designing the cryogenic fill system with a permeation loop that has less than a third the volume, but can collect $9600 \mathrm{Ci}$ of tritium into the system, requires additional methods of increasing pressure in the permeation loop be employed. The permeation loop can use a device like a gas intensifier as shown in Fig. 8. 


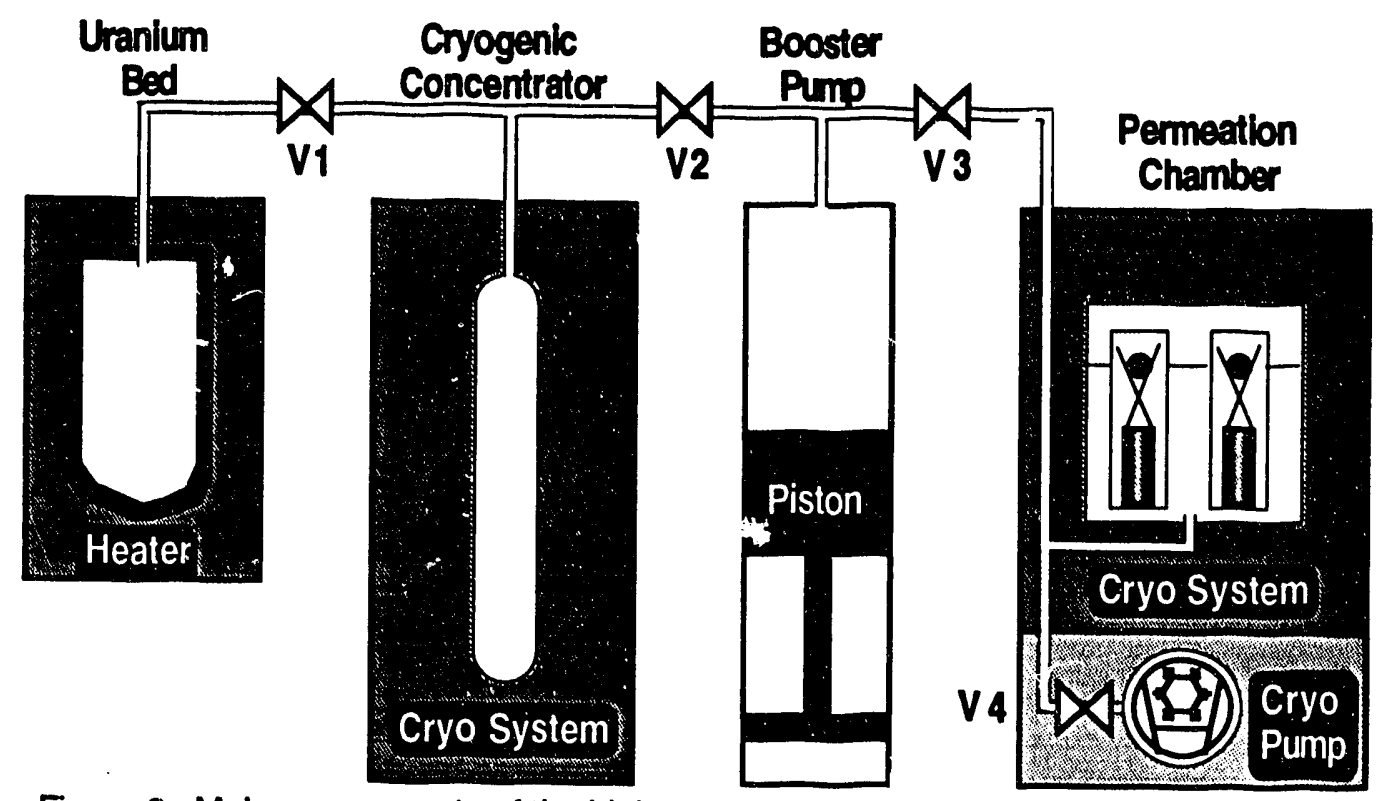

Figure 8. Major components of the high pressure cryogenic fill station. The cryogenic concentrator, booster pump and permeation chamber comprise the permeation loop

The volume of the condensation loop or cryogenic soncentrator is planned to be $10 \mathrm{cc}$ and the contact loop fill permeation chamber will be $5 \mathrm{cc}$ for a total of $15 \mathrm{cc}$ in the planned high pressure system.

\section{Valves}

The valves used in the UR/LLE tritium fill system permeation loop are Nupro bellows type valves. The bellows refers to the method in which the stem of the valve is sealed. A bellows is manufactured from thin, uniform tubing of a ductile metal and the convolutions allow flexibility during linear movement of the i alve stem (like an accordion). The bellows provides a metal barrier between the systern and atmosphere which is called a static seal. Bellows valves are used when greater reliability than packed valves is required (i.e., leakage past valve stem). This is why the valves were selected for the UR/LLE tritium fill system.

The design pressure of the UR/LLE tritium fill system was limited to $24,100 \mathrm{kPa}$ ( $3500 \mathrm{psi}$ ) because the Nupro HB series is the highest pressure rated bellows valve available. Significantly higher pressures are not possible with the bellows because of its thin-wall nature. Therefore, a packed valve with a sliding stem is required to achieve high pressure ratings.

A packed valve consists of $360^{\circ}$ seals around the stem. A packing nut is tightened and applies a compressive force on the packing, which deforms around the stem, to achieve a seal. A tight fit is required to produce a leak-proof valve in a tritium (hydrogen) environment. A packed valve will meet the requirements of the cryogenic fill system, but 
may involve significantly more maintenance than a bellows valve. Becaus: of this reason, it is recommended that the permeation loop design minimize the number of packed valves to reduce maintenance and possible locations of DT leakage. In addition, the packing can create substantial friction around the stem making pneumatic operation difficult. Therefore, the pneumatic actuators of the high pressure packed vaives will be large (e.g., $25 \mathrm{~cm}$.) and their location will have to be carefully planned to minimize the permeation loop system volume. It is planned to place the actuators in tubes on the outside of the glovebox to maximize working space inside the glovebox.

Manufacturers of high pressure valves are Autoclave Engineering Group, High Pressure Equipment Company, and Sno-Trik Company. All of them produce pneumatic-actuated high pressure valves with packing material compatible with Tritium (i.e., Buna-N or Grafoil).

\section{Condensation Concentrator/Condensation Loop}

The condensation loop needs to be carefully designed to minimize the internal volume. The high pressure $6.4 \mathrm{~mm}$ (1/4 inch) tubing in the permeation loop will have an inside diameter of only $1.6 \mathrm{~mm}(1 / 16 \mathrm{inch})$ resulting in a volume of $0.02 \mathrm{ml}$ per linear centimeter $(0.0031$ in $^{3}$ per linear inch) of tubing. Valves will also have a similar inside diameter. However, caution must be used when working with these small diameters near the condensation tube. The condensation tube should be designed so that its volume, or part thereof, is not blocked by the DT ice which initially forms in the tube. Small diameters are susceptible to icing.

The condensation tube must also operate at cryogenic temperatures for cryo-pumping the DT into the permeation fill permeation loop. The cryogenic temperatures are also used to pump the residual DT from the contact loop back into the condensation loop subsequent to a target fill. The wide temperature range the condensation tube experiences may require that it be thermally insulated from the remaining permeation loop system, primarily the high pressure valves whose packing may not be able to tolerate the extreme temperatures (warm or cold). Detailed heat transfer analyses are needed to determine what methods are necessary to isolate the temperature in the condensation tube.

\section{Permeation Fill Contact Loop}

The filling requirements for the permeation loop are for polymer targets. The high density fills will require that the targets he removed at cryogenic temperatures. However, emphasis should be placed on reducing the number of valves and tubing to minimize permeation contact loop system volume and possible locations of DT leakage. 


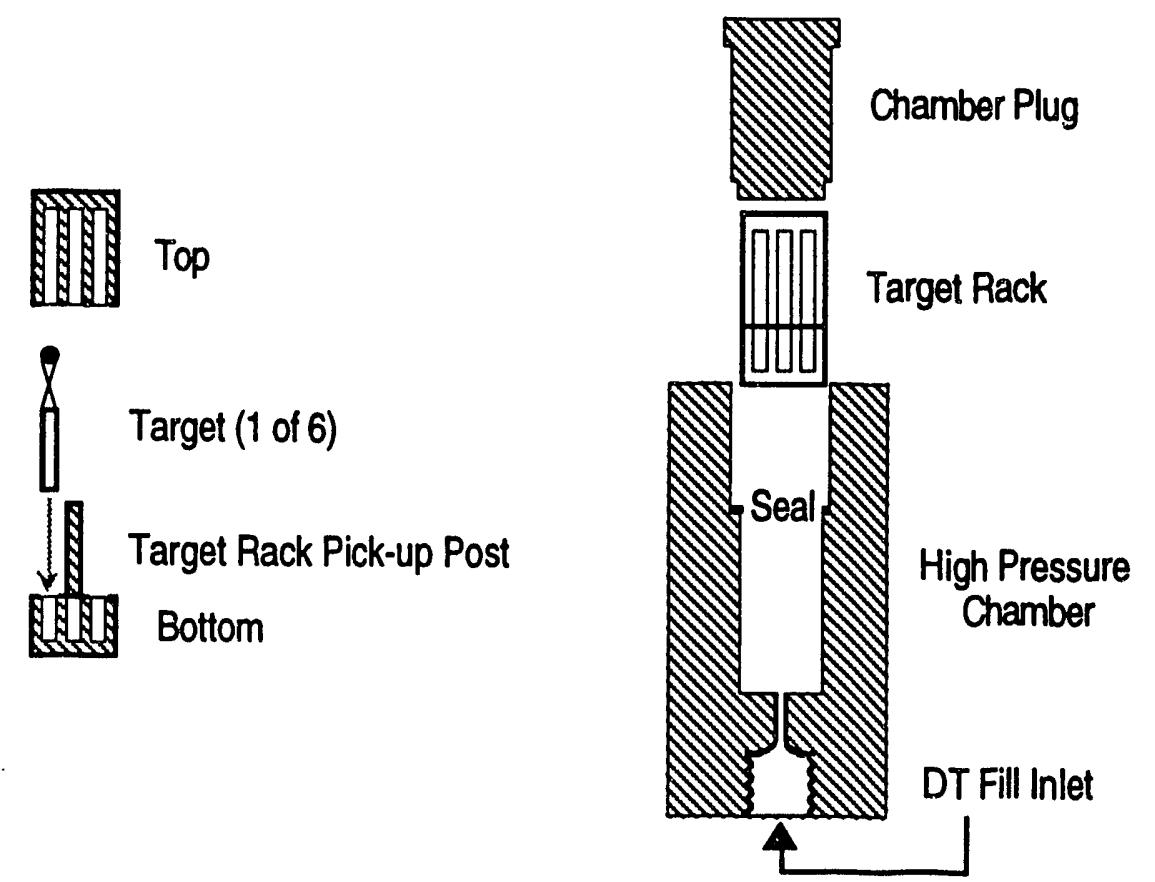

Figure 9. The permeation chamber will withstand high pressure, low temperature and have low volume

Because DT permeates through the polymer spheres rapidly at ambient temperature and it would be difficult to attach the target to a holding device at cryogenic temperatures, it will be necessary to mount them on their holders prior to filling with DT. An example of a target mounted on a holder is shown in Fig. 9. The plastic spheres are placed in the contact tube or permeation chamber along with their holders. Six spheres can be charged simultaneously. Space is provided in the holder or permeation chamber to place a small gripper and lift the target/holder out of the contact tube. Figure 9 shows a concept for a six target/holder insert and permeation pressure vessel. A heavy wall thickness is needed for the high pressure and the large inside diameter (required of the six target configuration). Design of the contact tube should be performed with the assistance of a high pressure equipment manufacturer. Their experience with experimental pressure vessels and sealing liquids and gases at various temperatures will be extremely helpful. Current design indicates a target rack of $10 \mathrm{~mm}$ diameter $\times 22 \mathrm{~mm}$ high. The permeation chamber vessel is $28 \mathrm{~mm}$ o.d. $\times 56 \mathrm{~mm}$ high. As indicated in Fig. 8, the inner volume of the cryogenic concentrator is $10 \mathrm{cc}$ and the inner volume of the permeation chamber is $5 \mathrm{cc}$ for a total of $15 \mathrm{cc}$ of dead volume. A system with only a cryogenic concentrator would have $15 \mathrm{cc}$ of dead volume. The cryogenic concentrator is allowed to warm to room temperature allowing DT to fill the $15 \mathrm{cc}$ in the system. By itself, this would only bring the pressure up to 590 atmospheres. By introducing a mechanical intensifier (compressing a $15 \mathrm{cc}$ volume to essentially zero), it can increase the pressure in the permeation chamber to 1500 atmospheres by decreasing the dead volume. 
With reference to Fig. 8, details of the steps and amounts of DT involved in 1500 atmosphere fills are shown in Appendix A.

In the UR/LLE tritium fill system, subsequent to a target fill sequence, the perneation loop is evacuated of DT by vacuum pumping prior to disconnect of the contact tube. This is accomplished at ambient temperature. In the cryogenic fill system, after filling, the contact tube will slowly be lowered to cryogenic temperatures to maintain target integrity. Condensation tube \#2 (Fig. 6) will also be lowered to cryogenic temperatures, but to lower temperatures than the contact permeation chamber (i.e., the contact cube is at $-20 \mathrm{~K}$ while the condensation tube is at 4.2 . ). This would provide a cryo-pumping action to evacuate the contact loor of all residual DT. Once this is accomplished, the contact loop would be isolated a: the condensation loop allowed to warm to ambient temperature. All DT would then be evacuated from the condensation loop by opening the valve to the uranium bed where the DT will be absorbed.

Pressure Differential Reducing Mechanism

The pressure differential reducing methods used in the UR/LLE design used both a palladium-silver ( $\mathrm{Pd}-\mathrm{Ag}$ ) membrane and trapped volume. The $\mathrm{Pd}-\mathrm{Ag}$ membrane is not applicable for a very high pressure system and will not be used in the new high pressure fill system. The trapped volume method seems to be the safe method of operation, but requires continuous op:ration of the valves resulting in their frequent maintenance.

An estimate has been made of the DT fill cycle duration:

Insert targets into holder and fill chamber

$30 \mathrm{~min}$

Insertion of fili chamber into fill system

$20 \mathrm{~min}$

System pump down

$30 \mathrm{~min}$

Heating of uranium hed trap and letting DT into calibrated volume

$30 \mathrm{~min}$

Time for DT permeation and stepwise pressurization to 1500 atmospheres

$240 \mathrm{~min}$

Cool down of fill chamber to cryogenic temperatures

$60 \mathrm{~min}$

Evacuation of excess DT from fill chamber and tubes

$10 \mathrm{~min}$

Transfer of target rack from fill chamber to transport device

$50 \mathrm{~min}$

Removal of iill chaznher for reloading

$30 \mathrm{~min}$

TOTAL cycle time

$510 \mathrm{~min}$

Assuming that up to $0.8 \mathrm{Ci}$ of tritium or $0.13 \mathrm{mg}$ of DT is contained is a target after a fill, the amount of tritium that will he used over a six month period (at 30 targets/month) is about $25 \mathrm{mg}$ of DT or $144 \mathrm{Ci}$ of tritiun.

\section{Pressure Enhancement Mechanism}

As was previously mentioned, the perneation loop will not be able to produce the desired maximum equilibrium pressure by a closed loop method (i.e., temperature control of a fixed volume). Therefore, a gas intensifier needs to be used to increase the final pressure without 
increasing the total working volume of DT. A possible gas intensifier system would have a work ratio of at least 10:1. The gas used for operating the gas intensifier would be helium and it would be a self contained system inside the glovebox. A pressurized container could hold enough helium gas to operate the intensifier numerous times before needing a recharge. The intensifier would te designed by a high pressure equipment manufacturer such as Autoclave Engineers. The actuators will be placed in tubes or pipes outside the glovebox to conserve working space in the glovebox.

\section{Tritium Confinement}

The glovebox used for containing the process system in the UR/LLE tritium fill station would seem suitable for the cryogenic fill system. However, to increase safety, the high pressure permeation loop will be isolated in a smaller containment box. It will be designed so that it could be opened to change the contact tube or permeation chamber and perform required maintenance on valves and fittings. A possible configuration might have all penetrations into the permeation loop containment through a single wall with the remaining box portion removable to allow servicing and maintenance. A more desirable configuration is to have a pass-through airlock large enough to allow' removal of any component that will be in the glovebox. The permeation loop containment box will be operated at slightly less than atmospheric pressure so DT leakage will be less likely to occur through the permeation loop containment wall. The permeation loop containment box will be connected to a purification system similar to the larger process component glovebox. It might be possible to use the same purification system, but the difference in glovebox pressure (i.e., the permeation loop is slightly negative while the process component glovebox is slightly positive) may require separate systems. The permeation loop containment box could also serve the purpose of a burst shield for the high pressure permeation loop system. Fortunately, the volume of the permeation loop is small and, therefore, would have a small energy level.

Exposure of the public from operation of the system must be zero. The UR/LLE tritium fill station design has the process component glovebox at slightly above atmospheric pressure to minimize contaminants leaking into the glovebox. This, however, raises some questions since DT leakage inside the glovebox will result in higher probability of leakage to the atmosphere outside the glovebox.

To minimize the possability of tritium leakage, the high pressure fill components to be supplied by GA will be enclosed in a containment vessel maintained sligh'ly below one atmosphere (a negative gauge pressure). A rupture of the contact permeation chamber would 
require leakage through two additional containment chambers before entering room 157 , which is a controlled area in itself.

An emergency backup power generator has been provided by UR/LLE to assure no loss of power will occur during tritium fill operations.

\subsubsection{Comparison to Design Requirements}

We believe that the proposed conceptual design for the cryogenic DT fill station meets all of the design requirements.

1) A combination of cryogenic and mechanical pressure intensification will be employed to fill empty targets with $D_{2}$ or DT gas to pressures up to 1500 atmospheres [requirement 4.4.1].

2) A closed cycle cryogenic cooling system will cool filled targets to below the triple point [requirement 4.4.2].

3) The fill station design requires only 0.79 grams of tritium. The remaining 0.21 grams reserve is sufficient to fill nearly 2000 targets, ten times more than would be shot in a six month period [requirement 4.4.3].

4) A triple containment environment with scrubbers, tritium cleanup and appropriate operating procedures will prevent the release of tritium to the environment [requirement 4.4.4].

5) Primary tritium recovery will be via connection to the enclosure of the high pressure fill station The existing UR/LLE tritium cleanup system for the glovebox will be used as the backup recovery system [requirement 4.4.5].

6) The UR/LLE DT fill station already has power failure protection through utilization of an emergency backup uninterruptible power supply [requirement 4.4.6].

\section{A.6. Problem Areas, R\&D Requirements and R\&D plans}

Some development work will be required. For example, evaluation of the most suitable packing materials for the high pressure valves need to be made. At present Grafoil and Vespel are being considered. Testing will be performed to determine the number of cycles the packing material can withstand before maintenance is required. Components like the fill chamber, tubing, valve bodies, etc. can be made from AISI 316 and the fill chamber gaskets from silver plated AISI 316 or indium for satisfactory performance.

At the present time, it is planned to employ a pass-through air lock system large enough to accommodate any component in the glovebox. 
Sometime during the life of the cryogenic fill system, it will be necessary to remove a panel from the process component glovebox, thus exposing the internals of the glovebox to the room. During the design of the cryogenic fill system, some consideration will be given to how the necessary decontamination of the gloveb ox can be accomplished so that operations such as panel removal are less difficult. Before final design, we will build a mockup of the glovebox and internal systems to assure that all equipment is accessible and can be operated efficiently and safely.

\subsubsection{Backup Approaches}

A study will be made to determine if it is practical to make the volumes in the DT permeation loop even smaller than currently contemplated to further reduce the amount of tritium at risk during a fill operation.

A search will be made for new improved high pressure valve designs to increase reliability and minimize the requirements for maintenance.

\subsubsection{Summary}

The DT fill system is based upon UR/LLE's existing DT fill station and utilizes all of its safety features. The high pressure DT fill system, with a permeation fill chamber capable of being cooled to cryogenic temperatures, is a low risk item whose proof-of-principle can be verified in a relatively short period of time. To further reduce the risk, the $D_{2}$ fill station will be constructed first to verify all aspects of operation prior to constructing the DT fill station.

\subsection{TRANSPORT TO STORAGE CRYOSTAT}

\subsubsection{Introduction}

This section details how targets are moved from the DT fill station to the storage cryostat and what apparatus is required. This apparatus is located in the DT Fill Station portion of Fig. 1. The bottom of the fill station cryostat contains a high pressure permeation cell. Before the targets can be removed from the cell, it must be opened to provide access to the target rack. A "cryogenic extension wrench" is used for this purpose. The cold trar sport tube system transfers up to six filled targets from the DT fill station cryostat to the storage cryostat.

\subsubsection{Design Requirements}

[4.6.2] In the situation when a filled target accidentally bursts, the transport method must not allow a release of tritium to the environment. 


\subsubsection{Options Considered}

An alternative system level approach was briefly considered. The entire target filling, transport, storage, layering, characterization and insertion systems would be located inside a single cryostat. This would minimize tritium handling problems. At the bottom of a cryostat, a cryogenic gantry crane would transport targets between the permeation cell, a storage rack, a layering and characterization apparatus and the insertion pylon. This approach piccludes the possibility to transport cryogenic targets off-site. It makes difficult the integration of both a $D_{2}$ and a DT fill station into the overall system. The glove box required around the DT fill station would have to be substantially enlarged to enclose the entire system. Much more equipment and many more controls would have to be operated inside the glove box. More equipment is inaccessible for repair. This also necessitates situating the DT fill station in the basement directly underneath the Omega Upgrade target chamber. Precision temperature control would have to be extended over a larger system.

\subsubsection{Baseline Design Description}

Targets will be transported cold from the fill station cryostat to the storage cryostat with a device known as a transport tube. The basic concept for the transport tube is a cryostat specially designed to have its bottom section detached and attached while inside another cryostat. Before the transport tube can be inserted into the fill station cryostat to remove the targets, the permeation cell must have its cap removed to provide access. The cryogenic extension wrench, a set of three concentric tools, is used. The outer two tools are wrenches for unscrewing the permeation cell cap. The inner tool is a mechanical pickup tool for picking up and removing the permeation cell cap and sub-cap from the fill station cryostat. Figure 10 illustrates the steps involved in transporting targets cold.

After the cap has been removed (Fig. 10a), the basic operation is to insert the transport tube into the fill station cryostat (Fig. 10b) and detach its bottom section (Fig. 10c). A mechanical tool in the transport tube grasps onto the target rack (Fig. 10d). The bottom section of the transport tube is re-attached, surrounding the targets (Fig. 10e). This establishes an independent cryogenic environment around the targets. The transport tube is removed from the fill station cryostat and taken to the storage cryostat. There the transport tube is inserted into the storage cryostat (Fig. 10f). The bottom section of the transport tube is again detached (Fig. 10g) and the transport tube lowered into the storage cryostat. There, the mechanical pick up tool deposits the rack containing the targets into a receptacle on an $X-Y$ translation stage at the bottom of the storage cryostat (Fig. 10h). The tube is raised, its bottom section re-attached (Fig. 10i) and removed from the storage cryostat (Fig. 10j) which will eventually be transported through the glovebox airlock and then to the layering module. 
The design of the transport tube has much in common with the cold transport system described by Alexander. ${ }^{3}$ However, that system was designed for the cold transport, in a vacuum, of a target cooled by thermal conduction through its suspension fibers. Anticipated layering techniques will require the thermal stability and isothermal environment provided by exchange gas cooling of the target. The main modifications to the above mentioned cold transport system design are adding an additional wall and using leak-tight seals to the bottom section. This includes using two, rather than one, walls in the bottom section. With these modifications, the transport tube will use helium exchange gas to cool the targets.

In order to operate successfully with the transport tube, the fill station cryostat and the storage cryostat include several features. At their tops, the cryostats have vacuum locks. These prevent air from entering and condensing on the targets when the cold transport tube is inserted or removed. The cryostats are divided into upper and lower cryogenic chambers. This prevents any thermal disturbance made while inserting the transport tube or removing its bottom section from propagating to the lower chambers and hence to the targets. The cryostats' upper chambers contain a device to remove or attach the transport tube's bottom section. Optical windows allow the alignment of the transport tube at the bottom of the cryostats to be actively monitored. The design features of the fill station cryostat and the storage cryostat are more fully explored in Appendix B.

${ }^{3}$ N. Alexander, J. Barden, Q. Fan and A. Honig, "A 1.5-4 K detachable cold-sample transfer system: Application to inertially confined fusion with spin-polarized hydrogen fuels", Rev. Sci. Instrum. 62, 2729 (1991) 


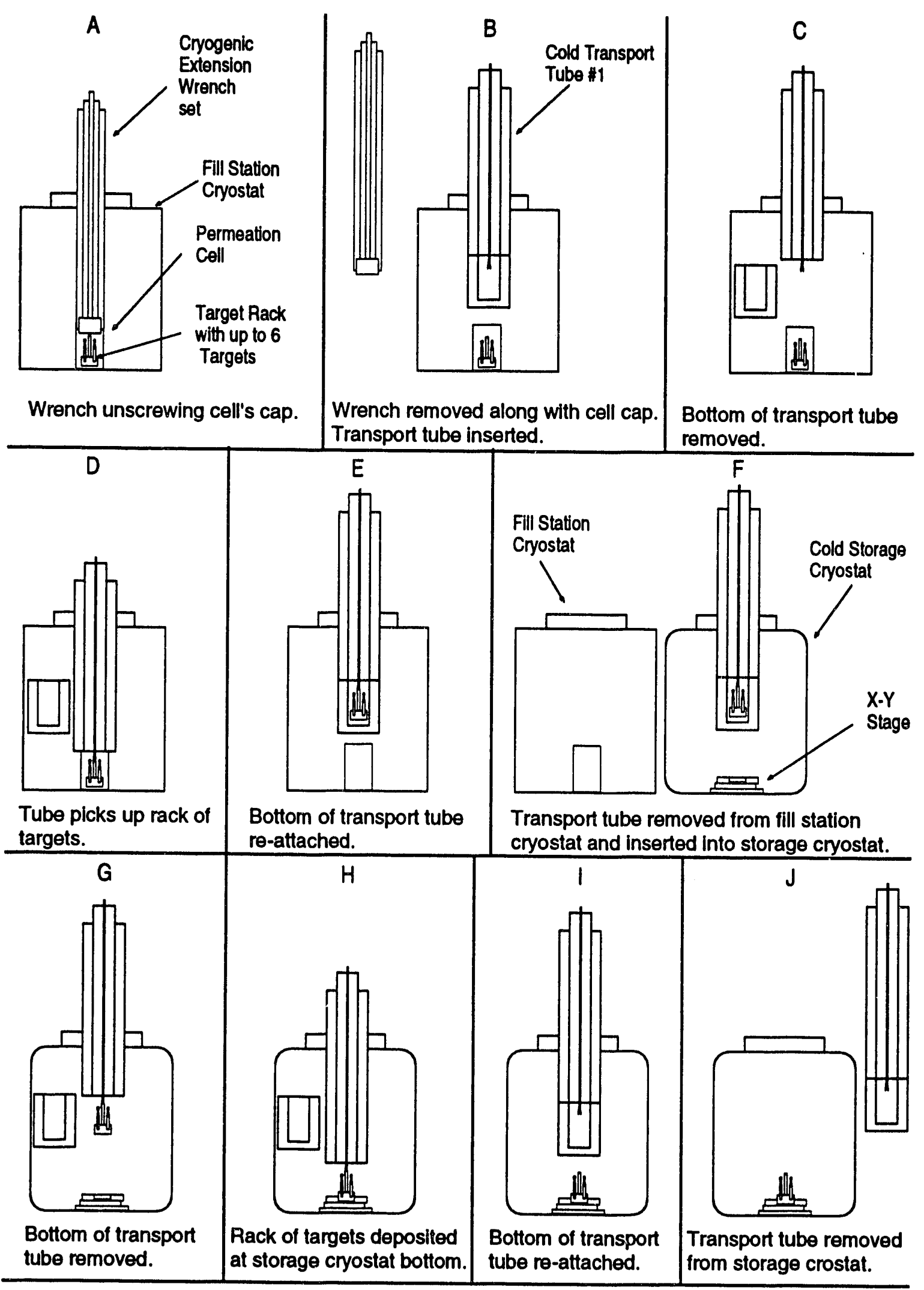

Figure 10: The steps for cold transport of a rack of targets from the fill station cryostat to the storage cryostat. 


\subsubsection{Comparison to Design Requirements}

The design requirements are met. The transport-to-storage cryostat operations are entirely within the glove box. In the event of accidental target breakage, all spaces in the fill station cryostat, storage cryostat and transport tube can be attached to a scrubber system and pumped on, removing the DT (requirement [4.6.2]).

\subsubsection{Problem Areas, $R \& D$ Requirements and R\&D plans}

There are several research and development areas. The exact design and material for the cryogenic seals need to be determined. Procedures need to be developed that insure that the permeation cell is not heated during the insertion of the transport tube into the fill station cryostat. The mechanisms and procedures for directing the transport tube directly over the target rack need to be developed. Procedures need to be developed to insure that the targets in the transport tube do not heat up when the transport tube is initially inserted into the storage cryostat. The use of closed-cycle refrigeration needs to be investigated. Several prototypes of the transport tube will need to be built to guarantee that they operate smoothly and reliably.

\subsubsection{Backup Approaches}

In the event that the materials and designs for the cryo-shroud seal cannot be made to work in the multiple cryogenic make and break seal mode described above, there is an operating procedure that allows the use of single make, single break seals made with indium o-rings. These have been used in a cryogenically breakable mode and were found to be leak tight against at least a $13 \mathrm{MPa}$ differential pressure ${ }^{6}$. The procedure for use of single use seals is more complex and time consuming.

\subsubsection{Summary}

The transport tube design will meet the design requirement. The development of this system appears straightforward. However, it requires a number of custom made parts so design, construction and testing over several prototypes will require a significant amount of time. An alternative, less desirable single use seal material is known to work for the cryo-shroud seals in the transport tube.

T. Norimatsu, H. Ito, C. Chen, M. Yasumoto, M. Tsukamoto, K. A. Tanaka, T. Yamanaka and S. Nakai, "Cryostat to provide a solid deuterium layer in a plastic shell for the Gekko XII glass laser system", Rev. Sci. Instrum. 63, 3378 (1992) 


\subsection{CRYOGENIC STORAGE}

\subsubsection{Introduction}

This section will describe the cryogenic storage system. In reality, the storage cryostat's primary function is as a transport mechanism between the fill station and the layering modules which are located at different physical locations at UR/LLE (Fig. 1). This system can also serve as a storage site if desired. It allows the accumulation of up to six filled, unlayered targets. Unlike the fill station cryostat, which is permanently attached to the DT fill station, the storage cryostat can be removed from the glove box of the DT fill station. If desired, cryogenic targets may be transported off-site in the storage cryostat. Compared with the fill station cryostat, the less constricted confines of the storage cryostat provide a more convenient access for the selection and pick up of a single mounted target. The storage cryostat requires several features to allow it to function in conjunction with the cold transport systems. These will also be described.

\subsubsection{Design Requirements}

[4.5.1] If a cryogenic storage system (for filled targets) is used, it must be able to withstand a complete loss of refrigerant without a release of tritium to the environment.

[4.5.2] If used, the cryogenic storage system must be able to hold at least six filled targets.

\subsubsection{Options Considered}

As mentioned in the previous section, an alternative system level approach was briefly considered; locating the entire target delivery system inside one cryostat. It was judged to be undesirable for the reasons previously mentioned. The decision to used closed-cycle vs. open-cycle (liquid cryogens) has not yet been decided. The open-cycle system is smaller and does not require connection to a power source. It does have the disadvantage of a limited hold time. Long term storage (most likely $\mathrm{D}_{2}$ targets) would require replenishing the cryogens on a regular basis or another storage system, most likely in the layering chamber, which will have a closed cycle refrigeration system 


\subsubsection{Baseline Design Description}

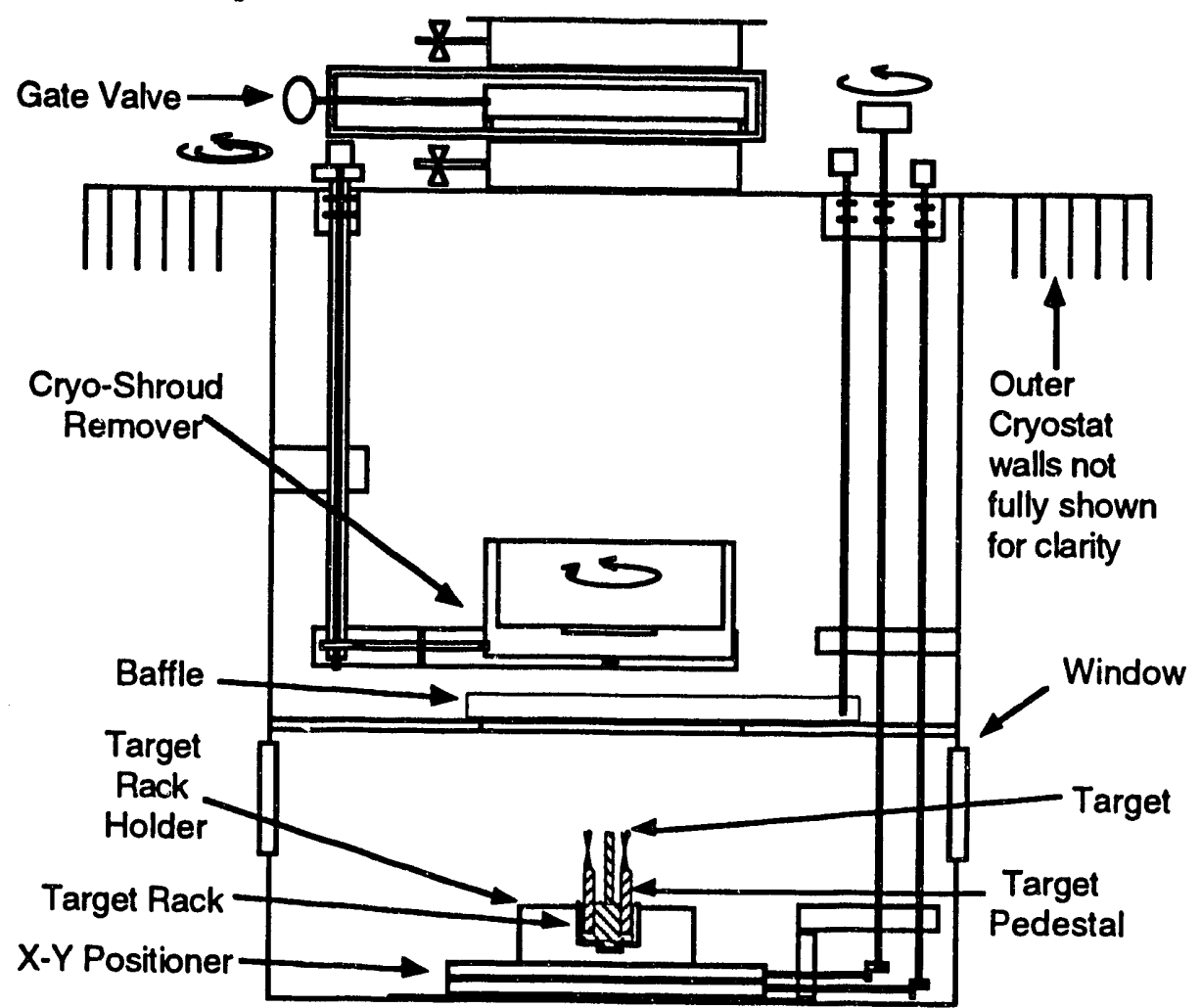

Figure 11. The storage cryostat showing the permeation cell.

The storage cryostat is illustrated in Fig. 11. The targets in the storage cryostat are cooled with helium exchange gas at $\sim$ one torr. At the bottom of the cryostat is a receptacle for the target rack. The receptacle sits atop an $X-Y$ translation stage used to maneuver individual mounted targets directly underneath a second transport tube (\#2). In order to operate successfully with the transport tubes, the storage cryostat includes several features. A vacuum lock prevents air from entering and condensing on the targets when the transport tubes are inserted or removed from the cryostat. It is divided into upper and lower cryogenic chambers. This prevents any thermal disturbance made while inserting either transport tube or while removing their bottom sections from propagating to the lower chamber and hence to the targets. The cryostat's upper chamber contains a device to remove or attach the transport tube's bottom sections (the cryo-shroud remover). Dual concentric shafts and an internal transmission allow the cryo-shroud to be swung into the center of the storage cryostat and allow independent rotation of the cryo-shroud remover's socket. The storage cryostat incorporates windows that allow actively monitored alignment of both transport tubes to the targets at the bottom of the cryostat. The design features of the storage cryostat are more fully explored in Appendix B. 


\subsubsection{Comparison to Design Requirements}

The design requirements are met. In the event of accidental target breakage, all spaces within the storage cryostat are made with volumes in excess of $4 \mathrm{cc}$. In the event that the storage cryostat warms to room temperature, this is sufficient volume for the DT fuel in six of the largest anticipated targets and the one torr helium exchange gas to have a pressure below one atmosphere. All spaces in the storage cryostat can then be attached to a scrubber system and pumped on, removing the DT (requirement [4.5.1]). The target rack holds six targets and fits into the storage cryostat (requirement [4.5.2]).

\subsubsection{Problem Areas, $R \& D$ Requirements and $R \& D$ plans}

There are several research and development areas. The exact design and material for the seal of the baffle between the upper and lower cryogenic chambers of the storage cryostat need research. Procedures need to be developed that insure that the targets are not heated during the insertion of either transport tube into the storage cryostat. The mechanisms and procedures for directing the transport tube directly over the a single mounted target need to be developed. A cryogenic X-Y translation stage will have to be developed. Closed-cycle vs. open-cycle refrigeration needs to be decided. Several prototypes of the storage cryostat will need to be built to guarantee that they operate together, smoothly and reliably.

\subsubsection{Backup Approaches}

In the event that a cryogenic $X-Y$ translation stage turns out to he impracticable, the X-Y stage and bellows can be mounted at room temperature at the top of the storage cryostat. This will still permit the alignment of the transport tubes to the targets.

\subsubsection{Summary}

The storage cryostat design meets design requirements. The develr $\mathrm{r}_{\mathrm{p}}$ ment of this system appears straight forward. However, it requires a number of custom-made parts, so design, construction and testing of several prototypes will require a significant amount of time.

\subsection{TRANSPORT TO LAYERING MODULE}

\subsubsection{Introduction}

This section details how a target is moved from the storage cryostat to the layering cryostat and what apparatus is required. Transport tube \#2 transfers a target from the storage cryostat to the layering cryostat. The cryostats into which transport tube \#2 will be inserted require certain features which will also be described. 


\subsubsection{Design Requirements}

[4.6.2] In the situation when a filled target accidentally bursts, the transport method must not allow a release of tritium to the environment.

\subsubsection{Options Considered}

As mentioned in the previous section, an alternative system level approach was briefly considered; locating the entire target delivery system inside one cryostat. It was judged to be undesirable for the reasons previously mentioned.

\subsubsection{Baseline Design Description}

Like the Transport to Storage module, targets will he transported cold from the storage cryostat to the layering cryostat in a transport tube. The design of the second transport tube (\#2) is identical to that of transport tube \#1, except that its mechanical pick up tool is designed to pick up a single mounted target rather than a target rack. Figure 15 illustrates the steps involved in transporting a single target to the layering apparatus.

Transport tube \#2 is inserted into the storage cryostat (Fig. 12a). The outer wall of the bottom section of transport tube $\# 2$ is cooled and then the bottom detached (Fig. 12b). The tube is lowered to the bottom of the storage cryostat. An X-Y stage containing the targets is adjusted so that an individual target is directly underneath the mechanical pickup tool of the transport tube. The mechanical tool in the transport tube slips over the target and grasps onto the target's pedestal mount (Fig. 12c). The transport tube is raised to a level in the storage cryostat that allows the bottom section to be re-attached, surrounding the target. The hottom section is reattached, establishing an independent cryogenic environment around the target (Fig. 12d). Transport tube $\# 2$ is removed from the storage cryostat and taken to the layering sryostat. There, it is inserted into the layering cryostat (Fig. 12e). The outer wall of the bottom section of transport tube $\# 2$ is again cooled and the bottom section detached (Fig. 12f). The transport tube is lowered to the bottom of the layering cryostat. There, the mechanical pick up tool deposits the target into a receptacle on a $z$-translation stage at the bottom of the layering cryostat (Fig. 12g). The tube is raised, its bottom section re-attached (Fig. 12h) and removed from the layering cryostat (Fig. 12i). The features required for successful transport to the layering module are the same as for the Transport to Storage module: vacuum locks, a removable bottom on the transport tube, etc. A much more detailed description of the design of transport tube $\# 2$ and its operation are included in Appendix C. 


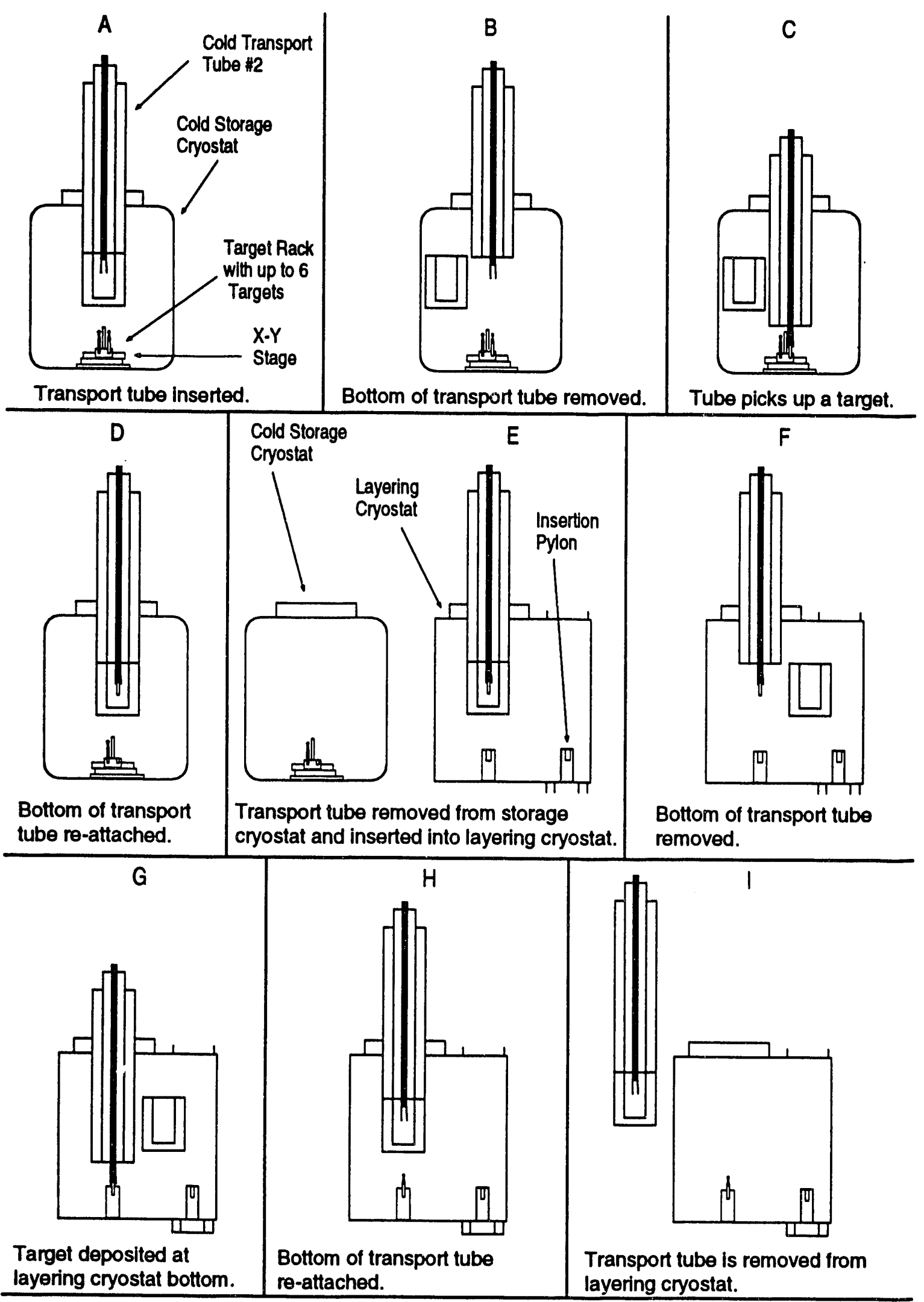

Figure 12: The steps involved in the cold transport of a target from the storage cryostat to the layering cryostat. 


\subsubsection{Comparison to Design Requirements}

The design requirements are met. The transport operations are entirely within the Omega target bay-a confined area. All spaces within transport tube $\# 2$ are made with volumes in excess of $1 \mathrm{cc}$. This is sufficient volume for the fuel in one of the largest anticipated targets and the helium exchange gas to have a pressure below one atmosphere should transport tube \#2 warm to room temperature. In the event of accidental target breakage, all spaces in the transport tube can be attached to a scrubber system and pumped on, removing the DT (requirement [4.6.2]).

\subsubsection{Problem Areas, R\&D Requirements and R\&D plans}

The problems and R\&D needs are identical to those of the Transport to Storage module. These include cryogenic seal development, temperature regulation, X-Y positioning and the question of closed-cycle refrigeration. Like the Transport to Storage transfer tube, several prototypes of the transport tube will need to be built to guarantee that they operate smoothly and reliably.

\subsubsection{Backup Approaches}

The backup approach to the Transport to Layering module is identical to the backup approach for the Transport to Storage module.

\subsubsection{Summary}

The transport tube meets the design requirement. The development appears straightforward. However, it requires a number of custom made parts so design, construction and testing over several prototypes will require a significant amount of time. An alternative, single use, less desirable seal material is known to work for the cryo-shroud seals in transport tube \#2.

\subsection{LAYERING}

\subsubsection{Introduction}

The layering technique will be the comerstone of the target delivery system. How the target is handled and characterized will be, at least to some extent, determined by the characteristics of the layered target. There are two hydrogenic fuels which are planned for use as Omega Upgrade cryogenic targets. The majority will be $\mathrm{D}_{2}$-filled capsules and the rest will be DT-filled. The layering technique is required to produce uniform and smooth fully dense cryogenic fuel layers. There is no clear-cut choice for the layering technique. Fuel layer thickness and constraints on the surface smoothness go beyond the limits of current target technology. This requires research, development and close interaction with the National Laboratories which are doing research in these areas. The ICF National Laboratories have the responsibility for specifying the appropriate layering method. 


\subsubsection{Design Requirements}

[4.7.1] The target delivery system will use a layering method(s) to be determined.

[4.7.2] The layering method(s) shall produce uniform linuid and/or solid $D_{2}$ or DT layers ranging between 10 and $100 \mu \mathrm{m}$.

[4.7.3] The design goal of the layering method will be to produce an inner surface finish better than $1000 \AA$ with a fuel mass uniformity, cuncentricity and sphericity all better than $2 \%$.

[4.7.4] The layering method must maintain the fuel layer characteristics until the target is shot.

\subsubsection{Options Consiciered}

Typical capsules currently used at UR/LLE are on the order of $250 \mu \mathrm{m}$ in riameter. These capsules can be filled with gas at pressures up to about 200 atmospheres. This produces cryogenic fuel layers on chie order of a few micrometers. The layering methods previously developed were for this category of targets. These included:

1) Fast Refreeze-One of the early methods which is useful for thin layers where the times for evaporation and sondezsation are short compared to the gravity induced sag time. The $100 \mu \mathrm{m}$ thick layers required by UR/LLE are too thick for this technique to work.

2) Thermal Gradient-Fiere the distribution of the fuel inside the shell is brought about by an anisotropic thermal environment. The temperature gradient produces a gradient in the surface tensiori and evaporative/cnndensate fluid flow. The fuel can be distributed around inside the capsule by adjusting the 3D temperature profile to produce a uniform thick layer. This technique, employing helium gas jets to generate a temperature gradient, has been used by UR/LLE in their previous cryogenic campaigns?

3) Beta-heating - This method is a "self layering" technique for thick, solid layers of $T_{2}$ or DT. The layer must be thick enough so that the energy from the beta-decay of tritium is deposited in the fuel layer. Areas where the fuel is thick will be warmer than areas where the fuel is thin. Fuel on the inner surface will evaporate from thick regions and deposit on thin regions. A steady state will eventually be reacheci where there is a uniformly thick fuel layer. This method could he suitable for the DT targets, hut not for $D_{2}$ targets.

\footnotetext{
${ }^{7}$ R. Q. Gram, «. D. Wittman, C. Immesnete, H. Kim, R. S Craxton, N. Sampat, S. Swales, G. Pien, J. M. Soures and $\mathrm{H}$. Kong, "Uniform liquid-fuel layer produced it. a cryogenic inertial fusion target by a time-dependent thermal gradient", J. Vac. Sci. Technol. A 83319 (1990)
} 


\subsubsection{Baseline Design Description}

As stated in the design requirements, the layering method is to be determined. However, to put together the conceptual design, a baseline method needed to be chosen. Furthermore, it had to be suitable for layering $D_{2}$ as well as DT targets. Our choice was the thermal gradient method with a quick freezing of the fuel to produce a solid layer.

The layering process involves using gas jets above and below the capsule (Fig. 13) to produce the thermal gradient. Helium gas flowing through the jets generates the thermal gradient necessary to distribute the fuel. The temperature of the jets is controlled by heaters on the jets. The lateral position of the sphere relative to the jets is adjusted until the layer is cylindrically symmetric. Then the jet temperatures are adjusted until spherical symmetry is achieved. When the layer becomes uniform, the heaters on the gas jets are turned off to quickly freeze the fuel.

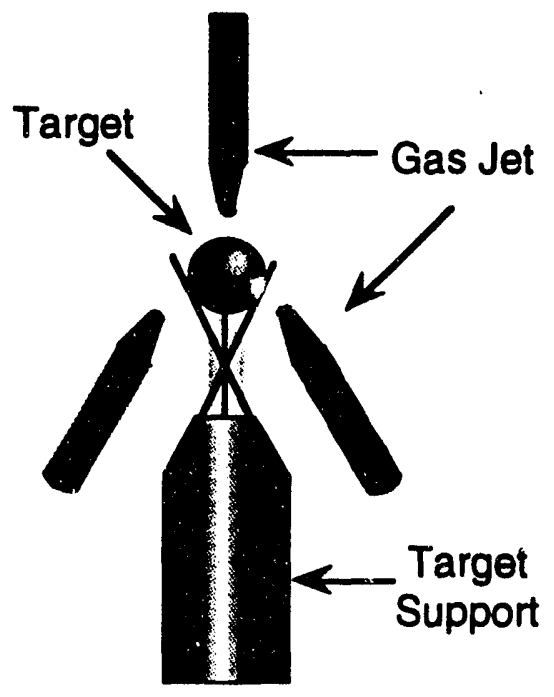

Figure 13 Thermal Gradient Layering

\subsubsection{Comparison to Design Requirements}

The thermal gradient technique is suitable for both $D_{2}$ and DT layering. With this technique, one can work with a liquid layer or freeze it and transport it as a solid layer. If the target can be kept in suitably temperature-controlled environment, a solid layered target will be easier to handle than a liquid layered target. The ability of thermal gradient layering (liquid or solid) to meet the design requirements is yet to be determined.

\subsubsection{Problem Areas, $R \& D$ Requirements and $R \& D$ plans}

The whole layering module is a problem area. We do not have a definitive layering method that is assumed to produce a uniform thick layer, nor to generate a sur' ace finish of $\sim 1000 \AA$. There is no characterization method currently in use that can measure inner surface finish. For DT targets, beta-heating always exists and should remove any low order $l$-mode nonuniformities if the solid layer is maintained near its melting point. However, inner surface smoothness is still unresolved. Hopefully, small angle neutron scattering studies to be performed at LANL will shed light on this matter. Beta-heating is not an option for the $D_{2}$ targets. 
We will assist layering development by:

1) Maintaining interactions with LLNL's cryogenics group to keep up to date on their progress, especially regarding their thermal gradient layering and solid layer experiments. This includes suggesting $\mathrm{D}_{2}$ layering experiments to be done at LLNL.

2) Maintaining interactions with the LANL cryogenics group to stay up to date on their DT experiments.

It is important to note that the timetable specifies that a layering technique be decided upon by December, 1993. That is only 15 months from now.

\subsubsection{Backup Approaches}

It is important at this stage to keep alternative layering methods in mind. Options would include:

1) Liquid layers-If a thick, uniform liquid layer could be made, but not frozen with a smooth inner surface, an alternative is to keep the layer liquid. The liquid layer should not have the high order $l$-modes of a solid layer-a definite advantage. The liquid layer would be difficult, if not impossib.e to maintain during transport to the target chamber in the presence of a gravitational field. It has been suggested that microgravity ${ }^{8}$ or free-fall techniques may be capable of producing liquid layer targets.

2) Beta-heating-For DT targets, beta-heating always occurs. This process is currently being researched. A variation of this process for $D_{2}$ has been proposed, but no calculations have been done. This "pseudo beta-heating" process would involve placing beta-decay sources around the target so that beta-particles travel into the fuel and are absorbed. The nontritiated fuel would then redistribute in the same fashion as DT or $T_{2}$.

3) If discharge-This is a technique being developed by the Japanese which could be used for layering $D_{2}$ as well as DT fuels. Here an rf plasma is used to redistribute the fuel through an evaporative/condensate process like thermal gradient heating, but heated from the inside. They have done a proof-of-principle using krypton as the fuel with liquid nitrogen cooling.

4) Magnetic field-assisted microgravity-Magnetic methods would be used to induce a magnetic dipole force in the liquid fuel layer that opposes gravity and allows the generation of a uniform liquid layer.

\footnotetext{
P. B. Parks and R. L. Fagaly, "Field-assisted microgravity for target fuel layering", to be submitted to J. Appl.
} Phys. 
5) Foams-If the fuel cannot support layers $100 \mu \mathrm{m}$ thick, then an aiternative is to provide a structure for that support. Here the porous layer of the foam capsules contains (supports) the fuel. By overfilling, one avoids problems with the rough inner foam surface. This is considered a last resort option.

6) Other possibilities include acoustical levitation and rotational refreeze.

\subsubsection{Summary}

We have chosen the thermal gradient method for the layering module. The layering method is one of the keys to the target delivery system. Significant development is needed to demonstrate the technique and there is technical risk that it will not meet the design requirements. We need to determine a layering technique as soon as possible. Other aspects of the delivery system (transport, characterization, insertion into the target chamber, etc.) will be affected by the choice.

The FY93 task statement gives the responsibility of specifying the method to the ICF laboratories. We will monitor their progress in layering techniques and support their efforts as is mutually acceptable by all parties.

After a layering technique is chosen, a design will be developed for this module and submitted to both UR/LLE and the laboratory responsible for determining the layering method. Upon design approval, the module will be constructed, tested, and integrated into the rest of the system.

\subsection{CHARACTERIZATION}

\subsubsection{Introduction}

How well one can determine that the layering method works depends on the quality of the characterization system. For Omega Upgrade targets there are more stringent requirements on the targets and, therefore, a need to push the limits of the characterization systems and develop new techniques. The new targets will require uniformity measurements at the $1 \%$ level. With the upgraded laser, the roughness of the target's surfaces has become an issue requiring quantitative measurements.

\subsubsection{Design Requirements}

[4.8.1] The target delivery system will use a characterization method to be determined.

[4.8.2] The characterization method must not deform the fuel layer until the target is shot.

[4.8.3] The characterization method must verify fuel homogeneity.

[4.8.4] The goal of the characterization method is to provide information on surface roughniness to 1000 Â. 
[4.8.5] The characterization method must provide information on fuel layer thickness, concentricity and sphericity at the $1 \%$ level.

[4.8.6] The final pre-shot characterization in the target chamber must determine the location of the target to $\pm 5 \mu \mathrm{m}$ and confirm the integrity of the fuel layer (if necessary). This is addressed in the target insertion section.

\subsubsection{Options Considered}

Significant effort has been put into characterizing targets. Techniques vary from categories such as direct imaging, classical interferometry, holographic interferometry to different types of methods within each category. Each has its own positive and negative aspects. The choice for Omega Upgrade was based primarily on what UR/LLE has found to be good for them and for which they have experience and confidence. There has not been as much effort with respect to opaque capsules. We are not aware of a characterization technique for opaque capsules with the required resolution.

\subsubsection{Baseline Design Description}

As stated in the design requirements, the characterization method is to be determined. However, to put together the conceptual design a baseline method needed to be chosen. Our choice was the Modified Mach-Zehnder Interferometer used at UR/LLE.

The UR/LLE interferometer combines the features of shearing and Mach-Zehnder configurations (Fig. 14). With this arrangement, the path lengths in the two arms can be adjusted. Moving mirror $\# 2$ allows it to be used as a shearing interferometer.

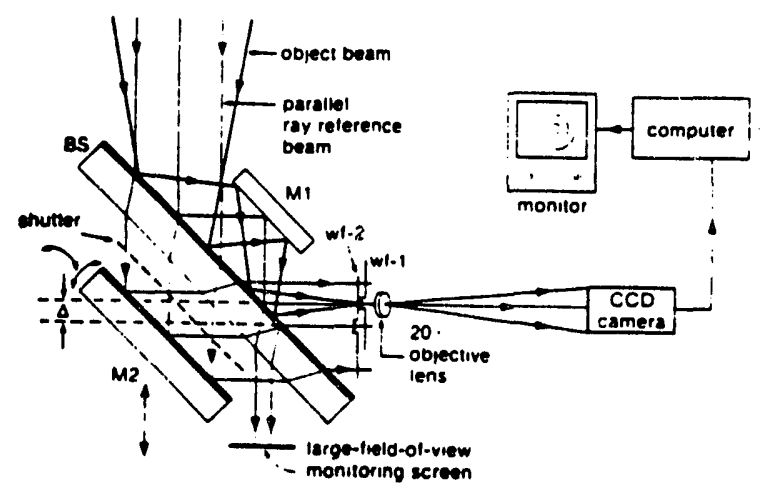

Figure 14 Schematic of Modified Mach-Zehnder Interferometer

The object and reference wavefronts follow the same paths with reduced sensitivity to vibration and air turbulence. Additionally, the reference wavefront is plane parallel and the direction of propagation can be widely adjusted in angle with respect to the object wave front. 
We intend to set up a system with three orthogonal views (figs. 52 and 53) which is an additional viewing direction from the system on the present Omega target system. This is the preferred viewing system for nonuniformities of order $l>1$. The orientation of the optics was chosen to give easy access to the target transport devices. The optical system also needs sufficient resolution to characterize the fuel layer. To estimate the $f$ number of the optical system, we used a resolution $(\delta \mathrm{x})$ of $1 \%$ for the $100 \mu \mathrm{m}$ thick layer. Using

$$
\delta x=\frac{0.61 \lambda}{\sin (\phi / 2)} \text { and } f \#=[2 \tan (\phi / 2)]^{-1}
$$

and a cone angle $(\phi)$ for $\lambda=0.48 \tilde{\partial} \mu \mathrm{m}$ (argon ion laser) of $35^{\circ}$, the $f$-number is $f 1.6$.

We will test the effects of possible thermal perturbations during insertion into the target chamber by returning a statistically significant number of targets to the layering/characterization system to check the integrity of the fuel layer. Although not stated as a specific design requirement, the targets will be opaque, unless a new shine-through barrier can be developed to replace the proposed aluminum barrier. This precludes doing online characterization with visible light. Our plan is to devise a procedure to repeatably layer transparent capsules and if the aluminum layer is not so thick that it affects the thermal gradients, then do a large number of transparent shells to statistically determine what must be done for "blind" layering of opaque shells.

\subsubsection{Comparison to Design Requirements}

The ability of Mach-Zehnder characterization to meet the design requirements is yet to be determined. As with the layering method, research and development is necessary to develop methods that meet the design requirements. The design requirements do give us the option of in-chamber characterization, if necessary. However, a system on the target chamber would probably have less resolution and would match to a preexisting template fringe pattern.

\subsubsection{Problem Areas, $R \& D$ Requirements and $R \& D$ plans}

The whole characterization module is a problem area. A system capable of meeting the design requirements has not been demonstrated. A Mach-Zehnder interferometry must be constructed to determine if it can characterize fuel layers at the $1 \%$ level. The basic MachZehnder offers acceptable resolution for thin fuel layers. The combination of a plane parallel reference wavefront and a thick fuel layer may cause too many fringes to be present. The use of a spherical wavefront has been suggested to overcome this.

Currently there is no method which can characterize the exterior surface roughness of a shell, let alone an inner cryogenic layer, at the $1000 \AA$ level. Assuming solid fuel layers, 
measurement of techniques to measure inner surface features at the $1000 \AA$ level will have to be developed. We will monitor the progress of LANL planned small angle neutron scattering experiments to see what inner surface smoothness beta-layered DT targets can achieve. We plan to attempt a modification of UR/LLE's interferometer system to handle large capsules at higher resolution levels. We will maintain close contact with the characterization groups at all the laboratories where work is going on in the development of ways to characterize large capsules and the capsule surfaces. This research is necessary for the development of larger capsules in addition to the need to characterize cryogenic layers.

There is also the problem of shine-through barriers. The need for a shine-through harrier leads to the requirement to develop a shell or coating that is opaque to $351 \mathrm{~nm}$ light from cryogenic temperature to plasma temperature. If opaque at visible wavelengths, this would preclude direct characterization of each shell during layering and prior to being shot. New, indirect characterization techniques would need to be developed. Two opaque characterization methods (ultrasonic characterization and nuclear magnetic resonance) will be examined at LANL during the next year. The resolution of these methods is unknown at this time. A less desirable alternative is to characterize a large number of filled shells to develop a data base that could be used to statistically infer characteristics of filled opaque shells.

GA has begun a program to develop new materials that would he transparent to optical wavelengths used in characterization. This work will continue at a low level of effort during 1993. Specific questions that must be answered include the opacity of the barrier $(90 \%$, $99 \%, 99.999 \%, . ?)$ and the surface absorption properties of the absorber-must it absorb at the surface (e.g., the first $\sim 0.1 \mu \mathrm{m}$ ) or can it be distributed through the shell? Whatever barrier that is used (fully opaque or transparent at visible wavelengths), it must be permeable to $D_{2}$ and DT near room temperature. An impermeable shine-through barrier would require unrealistically long permeation times.. Additional study on opaque barriers is needed to address this concern.

Finally, it is important to note that the timetable specifies that a characterization technique be decided upon by December 1993. That is only 15 months from now.

\subsubsection{Backup Approaches}

The primary focus of characterization technology in the community has been on optical characterization for the targets. There is no currently available method to characterize opaque targets. If the Omega Upgrade targets need to have aluminum coatings, then methods for characterizing them must be developed. Two methods to analyze opaque objects heing researched at LANL are: 
1) Ultrasonics-Here transducers are placed on a metal cell to drive resonance modes. The modes vary depending on the shape of the fuel inside the cell.

2) NMR tomography-This entails setting up a system using nuclear magnetic resonance imaging techniques to image the fuel layer inside the capsule.

At this time, it is unclear whether there is sufficient resolution with these techniques to be useful for characterizing Omega Upgrade targets.

We are also pursuing a polymer alternative to the aluminum layer. If one can develop a polymer which is transparent in the visible, yet opaque at the laser frequency, then it may be possible to apply it as a coating on the capsules. This gives one the ability to do characterization in the visible and retain the UV shine-through barrier.

\subsubsection{Summary}

We have chosen modified Mach-Zehnder interferometry for the characterization module. We need to develop and verify characterization technique as soon as possible. Other aspects of the delivery system (transport, insertion into the target chamber, etc.) may be affected by the choice. The FY92 task statement gives the responsibility of specifying the method to the ICF laboratories. We will monitor their progress in characterization techniques and support their efforts as is mutually acceptable by all parties. After a characterization technique is chosen, a design will be developed for this module and submitted to both UR/LLE and the laboratory responsible for determining the characterization method. Upon design approval, the module will be constructed, tested, and integrated into the rest of the system.

\subsection{TARGET INSERTION}

4.10.1. Introduction

After layering and characterization, the now-finished targets ate transported to the base of the chamber, possibly stored for a short period (avoiding ${ }^{3} \mathrm{He}$ buildup if a DT target), placed on the insertion mount, positioned at the laser focus, and fired. In this section we discuss this sequence of events and present a baseline design description as well as some alternatives.

\subsubsection{Design Requirements}

[4.6.1] Temperature excursions in the environment surrounding the layered target shall not exceed $\pm 0.2 \mathrm{~K}$.

[4.6.2] In the situation when a filled target accidentally bursts, the transport method must not allow a release of tritium to the environment. 
[4.9.1] The target insertion system must be compatible with the 60 Omega Upgrade laser beams and associated diagnostics.

[4.9.2] The target chamber insertion system for the Omega Upgrade laser must have an interlock to prevent the laser from firing if there is any possibility of damage to the laser or optics.

[4.9.3] The target insertion system must have greater than $\pm 5 \mathrm{~mm}$ positioning travel at the laser focus.

[4.9.4] The target insertion system must have better than $\pm 5 \mu \mathrm{m}$ positioning accuracy at the laser focus.

[4.9.5] The target insertion system must maintain the target to better than $\pm 5 \mu \mathrm{m}$ prior to the laser shot.

[4.9.6] Any refrigeration technique(s) that cool via gas conduction must not allow the gas pressure to exceed $10^{-4}$ torr at the tank center at the time of the laser shot.

[4.9.7] The target insertion system must be able to cycle targets in less than two hours.

\subsubsection{Options Considered}

Target insertion options center around where the layering and characterization work is to be performed, and where the target is inserted in the chamber. The choices are:

1) Layer, characterize, and insert at the top of the chamber.

2) Layer and characterize at a remote location and insert at the top of the chamber.

3) Same as above but insert at the bottom.

4) Do everything at or near the bottom of the chamber.

We recognize the importance of limiting activity on top of the chamber and also reducing the amount of hardware, particularly vibration-inducing hardware, at the top. For these reasons, we selected the last option with the exception of having a slow-moving gas shroud, discussed below, that extends from the top of the chamber.

\subsubsection{Baseline Design Description}

The most convenient location for the layering and characterization station is in the basement beneath the chamber hall ma in floor. While this will require cutting a hole in the main floor, it places the target and associated apparatus far enough away as not to interfere with target chamber operations. It is close enough to deliver to the insertion device while maintaining rigid temperature coutrol. The top and bottom ports are accessible for cryogenic target systems, althc...? it is assumed that other poris can be made available for 
room-temperature target insertion. This also means that the hardware and personnel operations should generally be limited to helow the main chamber support floor.

The insertion system must articulate to accurately position the target and hold the target in position. This requires that vibrations induced by any retractable thermal shrouds be adequately damped or delayed past the shot time. Figure 15 shows a schematic of the baseline design concept.

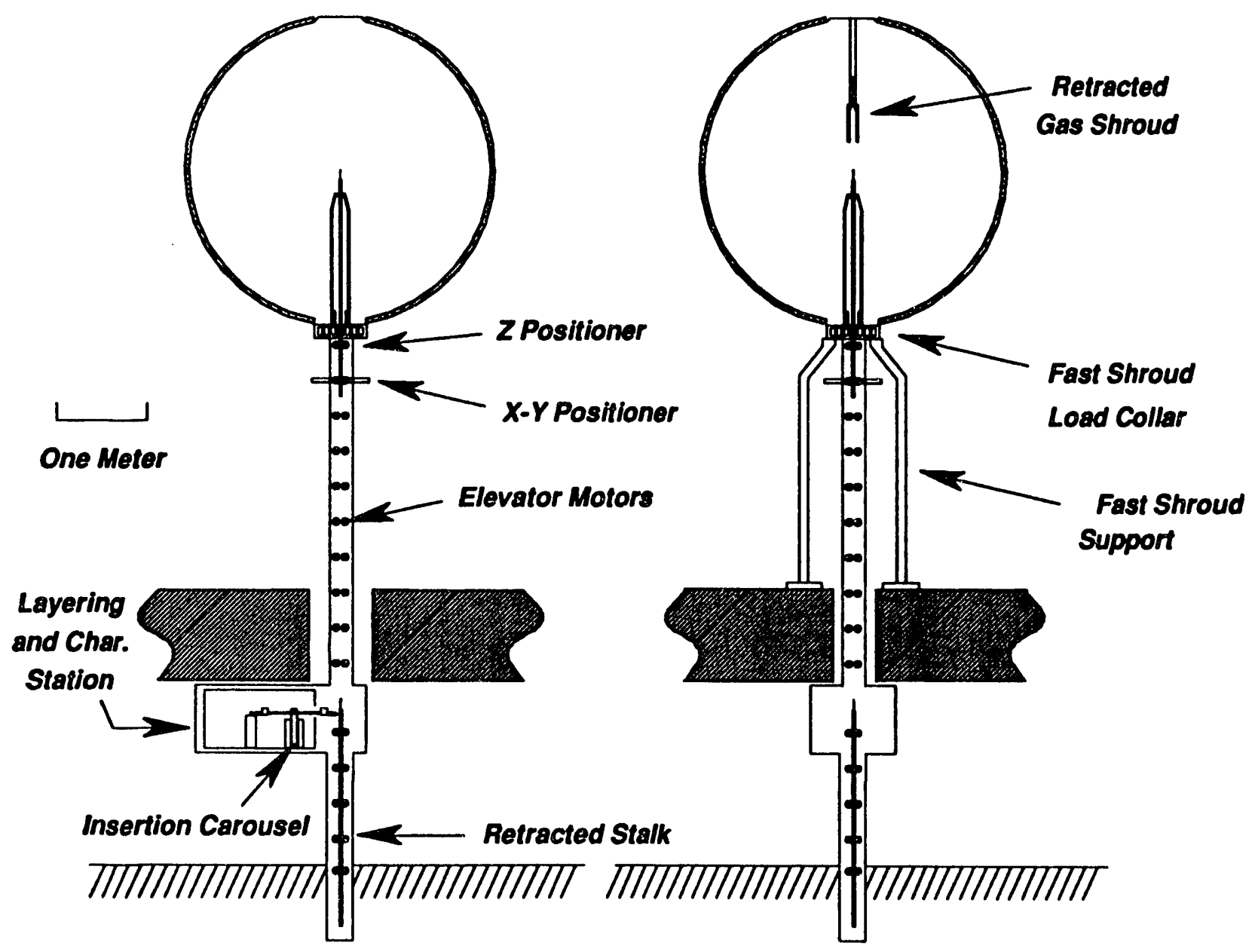

Figure 15. Target insertion baseline design concept.

The target insertion stalk descends to the level of the characterization station, picks up a target supplied by a rotary insertion carousel, and is elevated into position in the target chamber. Cryogenic, high-vacuum stepping motors are required for these maneuvers.

The topmost stepping motor also provides vertical positioning of the target. There may therefore be two clutched motors at this station, one with $\pm 5 \mathrm{~mm}$ travel and the other geared down for precision ( $\pm 5 \mu \mathrm{m}$ ) control. An X-Y positioner, also geared down, moves 
the bottom of the stalk. This places the $X-Y$ positioner in a more convenient location. However, it requires about five times greater resolution than if the $X-Y$ positioner were near the target. The Z-positioner provides the pivot point.

The long target insertion column is cooled by flowing helium gas at 1 torr and a nominal temperature of $16 \mathrm{~K}$. We assume that the target will not be disturbed by a gas flow that delivers a drag force less than $1 / 20$ of the weight of the target. Using a $1 \mathrm{~mm}$ diameter with a $20 \mu \mathrm{m}$ DT layer, a gas flow velocity of $5 \mathrm{~m} / \mathrm{sec}$ will satisfy this requirement. Thicker layers will further decrease the drag-to-weight ratio. Figure 16 shows the insertion channel in cross section. The central core, which contains the target and its insertion stalk, is about $10 \mathrm{~cm}$ diameter by $6 \mathrm{~m}$ long. It has a $77 \mathrm{~K}$ liquid nitrogen heat barrier and the core is further insulated by a polished $77 \mathrm{~K}$ surface having an emissivity of 0.05 and multiple layers of superinsulation.

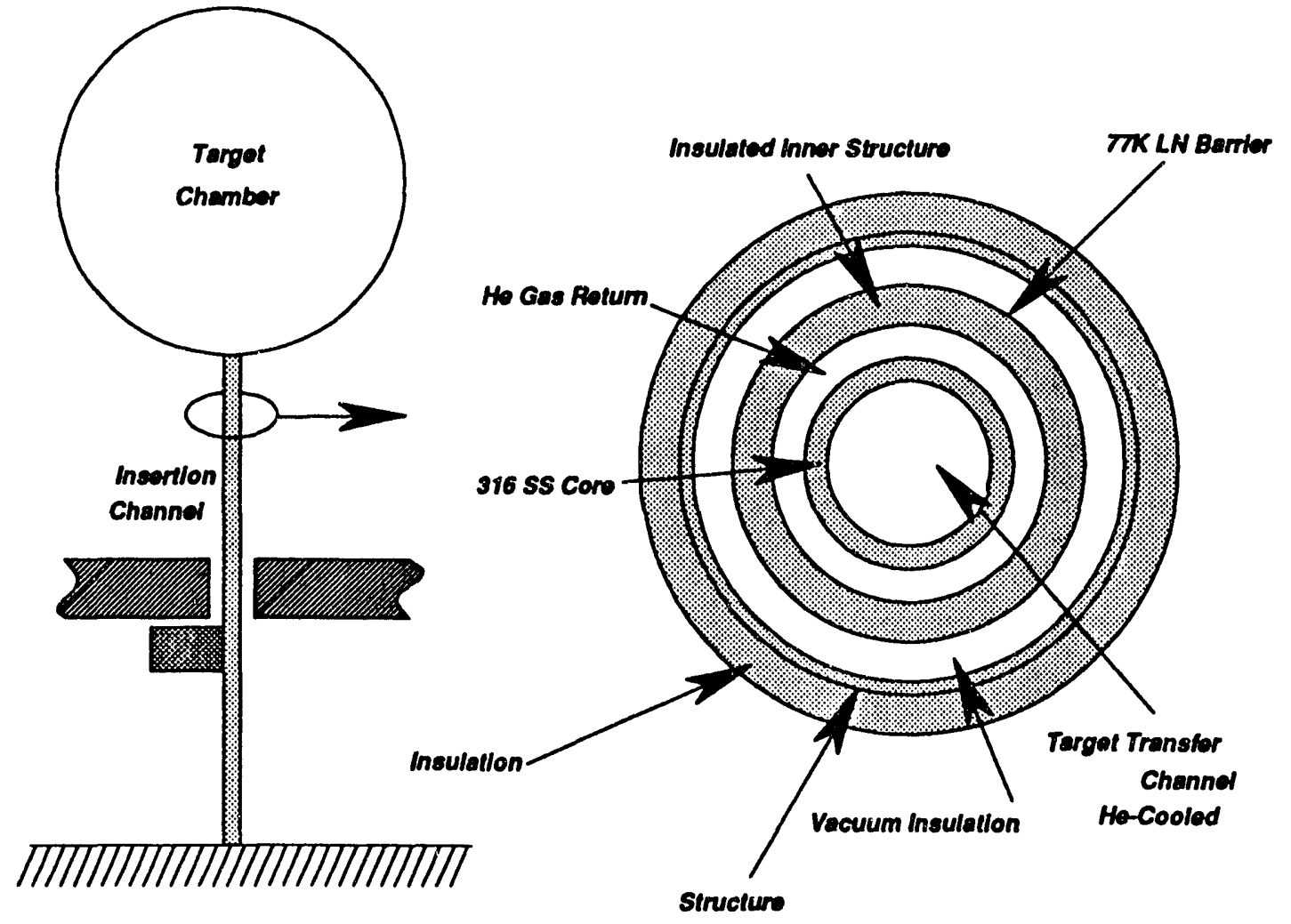

Figure 16. Cross-section of cryogenic target insertion channel.

The target must be maintained within one kelvin of the insertion temperature (c.f., $\$ 4.10 .5$ ) while it is at the center of the chamber. To ensure this, we have adopted a dual shroud protection system. One provides a gas seal, and the other provides protection from the $300 \mathrm{~K}$ chamber radiation. The gas seal shroud is attached to the top of the chamber and is slowly retracted a couple of seconds before the shot. This limits chamber vibration and allows time for the gas coolant to vent into the chamber and be partially pumped. Because 
the radiation shroud does not have to also seal the exchange gas, it can be made quite small and lightweight. It should be easily retracted in about $25 \mathrm{msec}$. The $300 \mathrm{~K}$ blackbody radiation heats a minimum-sized target at a rate of $20 \mathrm{~K} / \mathrm{sec}$ initially, giving a temperature rise of about $0.5 \mathrm{~K}$ in that interval.

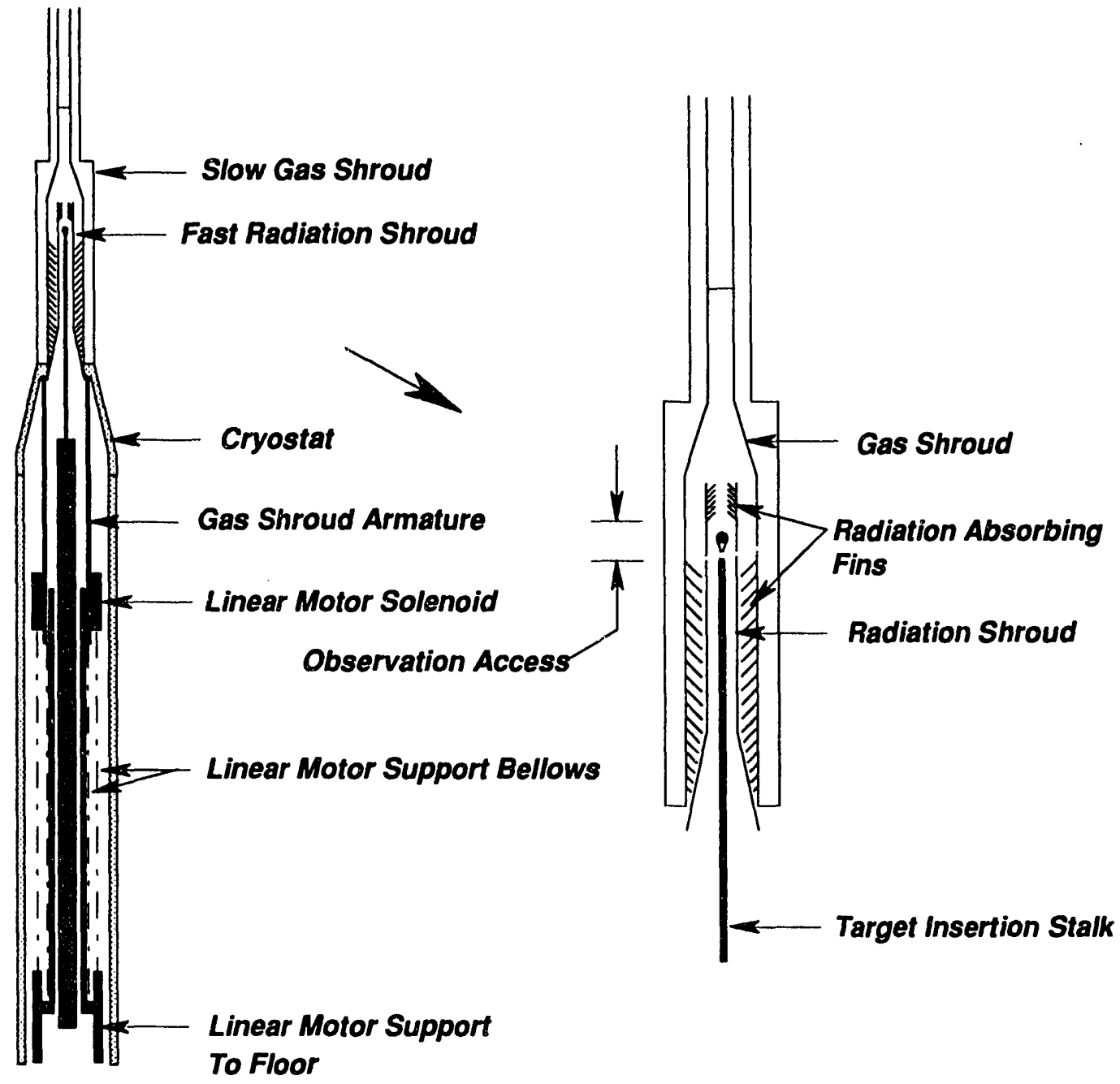

Figure 17 Dual shroud system for protecting target after chamber emplacement.

Figure 17 shows the configuration of these shrouds. Although not shown, gold-coated sapphire observation windows will allow the target to be portioned within $\pm 5 \mu \mathrm{m}$ of the focus of the Omega Upgrade laser beams. The window's gold coating will prevent thermal radiation from significantly heating the targets. The gas shroud forms a seal against the static cryostat. High emissivity fins absorb chamber radiation and, in the time interval of interest, reradiate it at very low temperature. Absorption of chamber radiation is minimized by having low $(0.05)$ emissivity outside surfaces. The main contribution to target heating turns out to be the $2 \%$ of the chamber radiation that is reflected from the fins. As seen in the figure, there is an area devoid of fins for observation of the target. In the two seconds 
between retracting the gas shroud and retracting the radiation shroud, the gas shroud temperature rises to no more than $50 \mathrm{~K}$ from $300 \mathrm{~K}$ black body radiation. The target heating rate during this interval, from the combination of re-radiation plus reflection, is about $0.15 \mathrm{~K} / \mathrm{sec}$ and is uniform throughout the target within $0.05 \mathrm{~K}$. After 2 seconds, target heating is about $0.3 \mathrm{~K}$.

The fast shroud is retracted by a linear induction motor which is mounted on bellows to dampen vibration to the target. The required travel is about $10 \mathrm{~cm}$ to get the shroud into the $26^{\circ}$ siladow region (see Fig. 18). For a $25 \mathrm{msec}$ retraction time, the shroud acceleration is a modest 32 g's.
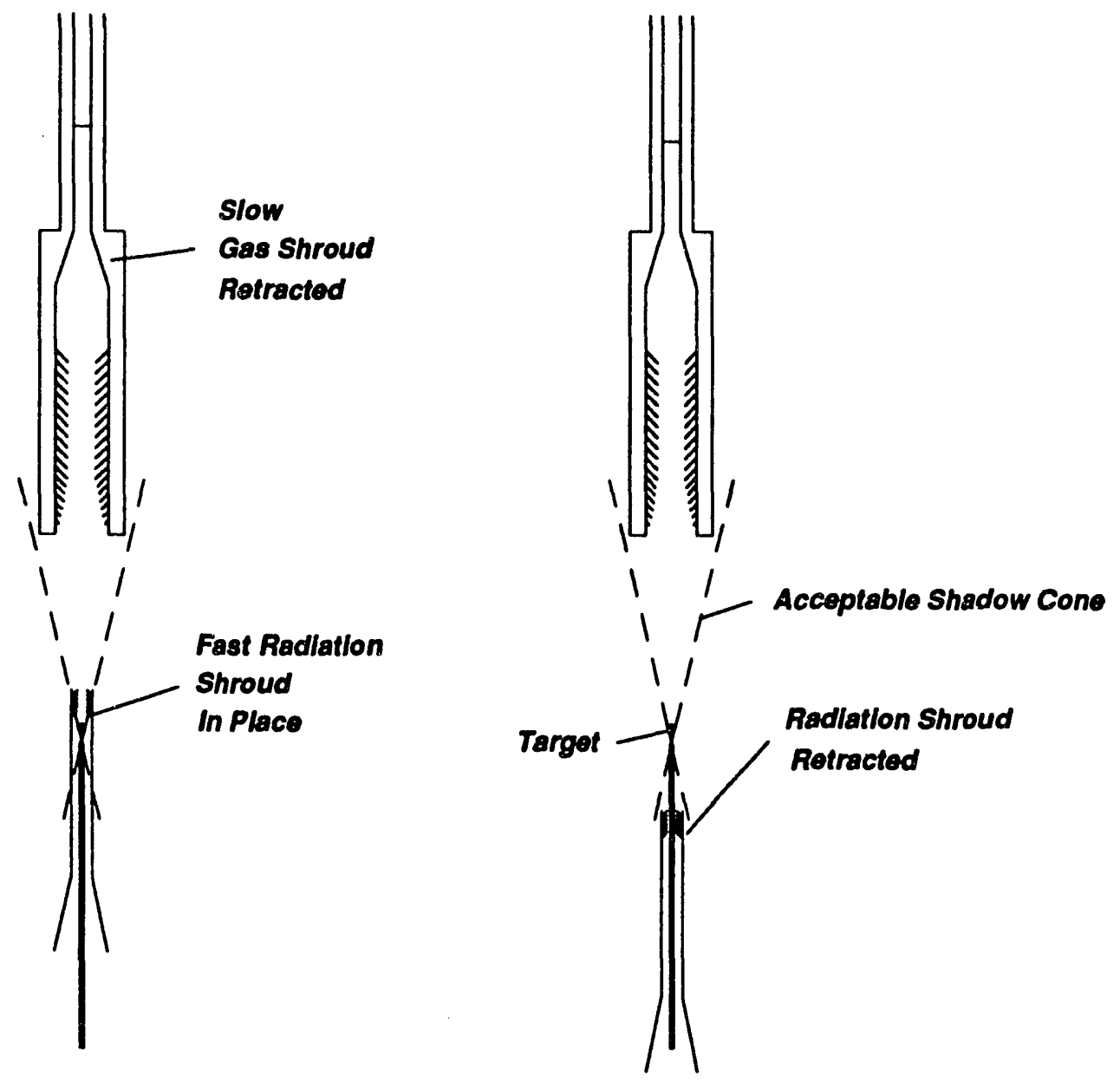

Figure 18 Motion of gas and radiation shrouds.

The motor solenoid is ballasted if required so that its mass is much larger than the shroud/armature assembly. Then, even though the system center of mass doesn't move, the shroud will be displaced much more than the solenoid. To further isolate the target 
from vibration, the bellows are attached to a support that is mounted separately to the chamber hall floor. The mechanical attachment to the chamber is flexible and provides mainly vacuum and thermal barriers. A smooth acceleration contour will also minimize vibrations.

The shrouds and insertion system will contain less than one liter of helium at a pressure of one torr and temperature of $15 \mathrm{~K}$. When vented to fill the target chamber, which has a free volume greater than 15,000 liters, the gas pressure prior to heating by the chamber walls will be $6 \times 10^{-5}$ torr. While this will increase by heating, there will also be helium removed by chamber pumping during the two second interval between shroud retractions. Separate pumping can be performed on the shroud gas prior to retraction to lower chamber pressure even further.

\subsubsection{Comparison to Design Requirements}

We believe that design requirement [4.6.1]-Temperature excursions in the environment surrounding the layeied target shall not exceed $\pm 0.2 \mathrm{~K}$ can be relaxed to at least $\pm 1 \mathrm{~K}$.

The primary reason for this design requirement is to preserve the integrity of the target between final characterization and the shot. Any target insertion system must not cause a change in target characteristics during the interval between the end of characterization and firing the shot. This in general requires that:

1) For a solid layer, the temperature remains below the triple point ( $19.79 \mathrm{~K}$ for $\mathrm{DT}$ and $18.73 \mathrm{~K}$ for $\mathrm{D}_{2}$ ).

2) The differential strain remains in the elastic range, i.e., below $0.2 \%$ at temperatures above $11.6 \mathrm{~K}$ dropping to $0.1 \%$ at $1.4 \mathrm{~K}$. No data exists to interpolate between 1.4 and $11.6 \mathrm{~K}$. We assume for the moment that the yield strain ${ }^{9}$ remains at $0.2 \%$ down to at least $6 \mathrm{~K}$.

3) The acceleration loads must be low enough to prevent shaking the DT ice layer loose from the shell. We assume anything below $0.25 \mathrm{~g}$ 's is acceptable $\left(1 \mathrm{~g}=9.8 \mathrm{~m} / \mathrm{sec}^{2}\right)$.

P. C. Souers, Hydrogen Properties for Fusion Energy (University of Califomia, Berkeley, CA 1986) p.85 


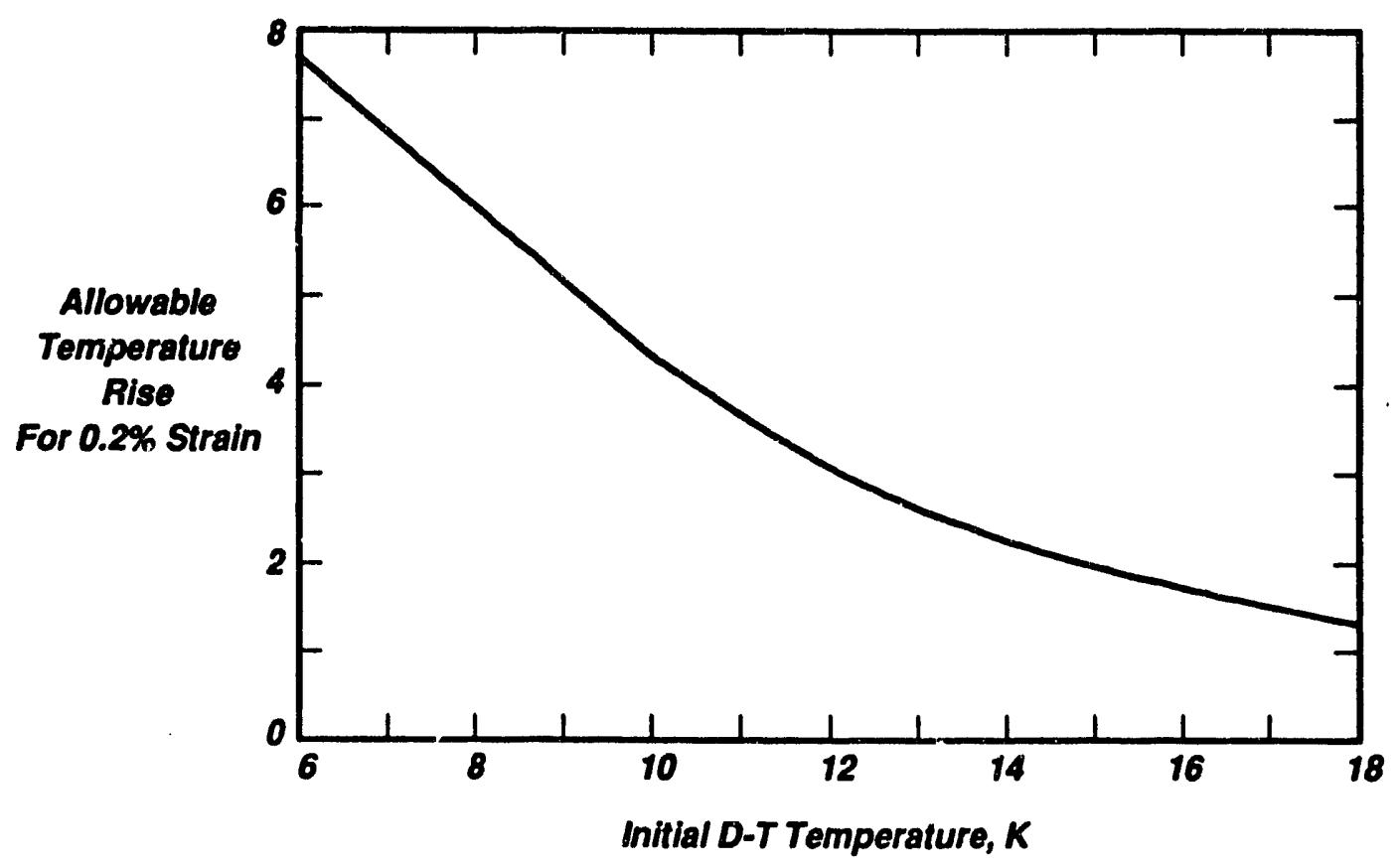

Figure 19 Allowable temperature rise in DT ice without exceeding elastic limit.

The second criterion establishes the allowable temperature cycling prior to the shot. It is assumed that surface morphology will remain acceptable if the stress in the DT ice does not exceed the yreld point. From Fig. 19, it " suld appear that there is greater tolerance to heating at the lower temperatures. However, this is not necessarily the case because heat capacity is also reduced at the lower temperature. Nevertheless, as seen in the figure, the allowable temperature rise never falls below $1.0 \mathrm{~K}$. We therefore recommend this value as our design criterion regardless of initial temperature, knowing that some overshoot would be acceptable. This criterion is further conservative for two reasons:

1) Thermal stress is given by $\frac{1}{2} \varepsilon \alpha \Delta T /(1-\mu)$ for rigidly fixed objects. Here $\varepsilon$ is the elastic modulus, $\alpha$ is the thermal expansion coefficient, and $\mu$ is Poisson's ratio. Thermal strain is simply $\alpha \Delta \mathrm{T}$. Thermal stress could be as much as half of that which would occur from an externally applied strain of equal magnitude.

2) For ordinary materials at least, allowable thermal stresses are typically double those that are externally applied. This is mainly because only the planes at the temperature extremes see those stresses. 
The result of this is a $1 \mathrm{~K}$ temperature excursion limit may be conservative by at least a factor of four, and even more at temperatures below $18 \mathrm{~K}$. Based on this analysis, we believe that design requirement [4.6.1]-Temperature excursions in the environment surrounding the layered target shall not exceed $\pm 0.2 \mathrm{~K}$ can be modified to at least $\pm 1 \mathrm{~K}$. The exact allowable temperature excursions will need to be confirmed experimentally.

Both shrouds retract far enough to permit full illumination of the target by the laser beams. The dimensions and withdrawal distances of the shrouds are such that they will not interfere with any of the laser beams or diagnostics (requirement [4.9.1]). The insertion stalk will utilize an $X-Y$ translation stage to meet requirement [4.9.3]. Two-axis view ports allow target positioning to within $\pm 5 \mu \mathrm{m}$ (requirement [4.9.4]). The port allows for an optical interlock to ensure the target is positioned properly before commencing the shot sequence (requirement [4.9.2]). Vibration from this shroud is well-isolated from the target by bellows attachments, independent mounting, and load contouring (requirement [4.9.5]). The gas shroud is removed about two seconds before the shot, allowing any residual gas to dissipate into the $\sim 15 \mathrm{~m}^{3}$ volume of the target chamber (requirement [4.9.6]). Although not demonstrated, we helieve that this system should permit the firing of DT targets every two hours (requirement [4.9.7]). Although not a design requirement, with both shrouds retracted, room-temperature targets can be inserted at the laser focus from another location on the chamber.

4.10.6. Problem Areas, $R \& D$ Requirements and $R \& D$ plans

Expected areas requiring some development are:

1) Precision linear and rotary stepping motors operating under vacuum and temperatures as low as $10 \mathrm{~K}$.

2) Dealing with the long $(6 \mathrm{~m})$ vertical travel of the insertion stalk while a target is attached.

3) Assuring that the radiation shroud retraction does not shake the target.

4) Minimizing the thermal radiation load from the shroud observation ports

5) Assuring that the firing interlock is fail-safe.

6) Continued concern over the mass perturbations on the target due to the tripod mount.

We don't foresee any significant problems with these concerns; nor is any lengthy deveiopment expected. These are mainly design issues that simply require more time to resolve. We will be investigating the first iscue immediately. The second and sixth are the 
motivations for alternate designs discussed below. In total, we expect these concerns to be resolved on the conceptual level in a few months.

\subsubsection{Eackup Approaches}

In this section we discuss a second target transport approach that eliminates the long travel path the insertion stalk with target must travel. We also discuss two alternate target injection schemes that both involve free-fall and eliminate mass perturbations entirely.

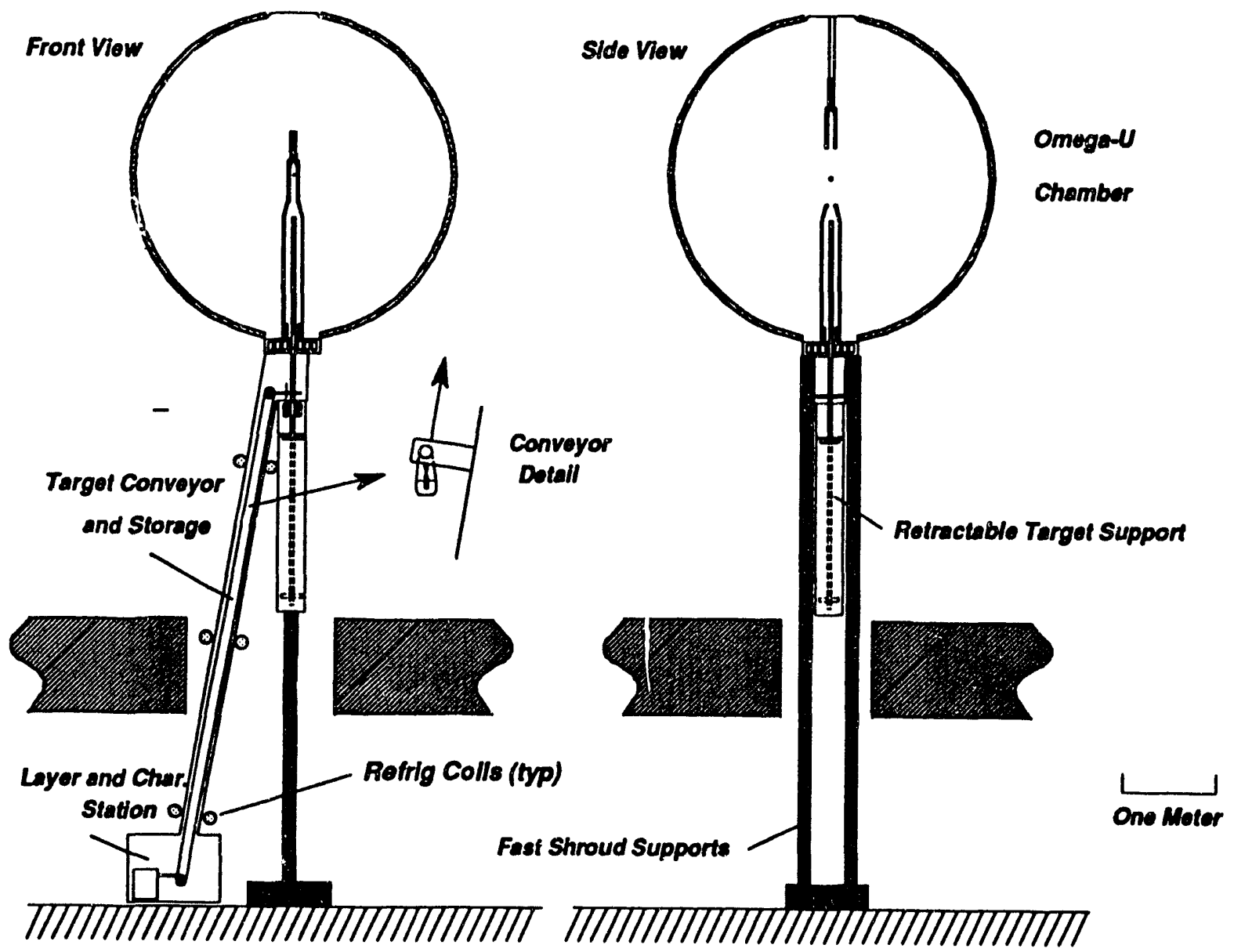

Figure 20. Alternate target transport using a conveyor.

\section{Conveyor Transport.}

It is inconvenient to have to lower the insertion stalk several meters using precision drives, cut a hole in the basement floor, and elevate the layering and characterization station to the ceiling of the basement. If a separate, relatively low-tech conveyor system were used, these problems can be eliminated. Figure 20 shows the concept. Targets are placed on a conveyor, somewhat resembling a chair lift, by a rotating carousel. The conveyor

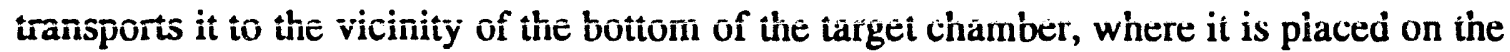


insertion stalk by another carousel. The insertion stalk travel is reduced to slightly more than the chamber radius. As with the baseline design, slowly-flowing helium gas keeps the target cool during transit.

The conveyor can also provide short-term storage for targets. It allows more flexibility in placement of the layering and characterization station, and reduces insertion stalk travel. It does, however, add the complexity of another transport mechanism, and a second insertion carousel. Further study and discussion are required to establish the relative merits of the two concepts.

\section{Free-Fall from Top of Chamber}

A totally different approach would be to eliminate the tripod mount and drop the target from the top of the chamber. The target must be protected from the chamber $300 \mathrm{~K}$ radiation. To do this, we suggest dropping it is a cryogenically-cooled tube that extends to within $10-15 \mathrm{~cm}$ of the shot point. After falling $\sim 2$ meters, the target velocity is $\sim 6.3$ $\mathrm{m} / \mathrm{sec}$ and covers the remaining $15 \mathrm{~cm}$ in $24 \mathrm{msec}$, the same exposure time as in the baseline design. Sensors, possibly fiber-optic, and lateral positioners would be placed inside the tube. The positioners could be either electrostatic or gas puff. At $6 \mathrm{~m} / \mathrm{sec}$, the \pm 5 $\mu \mathrm{m}$ position tolerance translates to about $1.6 \mu \mathrm{sec}$ uncertainty in firing time. This appears to be an acceptable tolerance. In addition to the elimination of any mass perturbations in the target, this concept has appeal because it completely eliminates the retractable shrouds. Concerns center around the ability to position the target laterally, and the fact that the target cannot be observed in place prior to the shot.

With about $0.6 \mathrm{sec}$ of free-fall time, there is the intriguing possibility that a target containing liquid $\mathrm{D}_{2}$ or DT may layer itself by wetting during the fall. This introduces the possibility of testing targets in a different parameter regime, in particular, one where the DT gas in the target core is above the triple point pressure $(-3 \mathrm{~atm}$.). The use of liquid layers is a backup method of layering (c.f., §4.8).

\section{Free-Fall from the Radiation Shroud}

The positioning concerns associated with dropping targets can be mitigated by using the radiation shroud from the baseline design as a "golf tee". This tee would be quickly pulled down and out of the way to ensure purely vertical, nonrotary motion. Such a device was constructed utilizing a graphite support pedestal ${ }^{10}$ The low initial acceleration of the graphite support pedestal (and accompanying electrostatic forces) produced focal-zone dwell times of $\sim 0.33 \mathrm{msec}$ as opposed to the desired $\sim 2.5 \mathrm{msec}$. The use of a grounded

\footnotetext{
${ }^{10}$ KMS Annual Report pp. 1-61 to 1-65 (1977)
} 
support pedestal should eliminate electrostatic forces. It allows precise target positioning. Based on present technology, the support pedestal could be made to retract $10 \mathrm{~cm}$ in 10 msec (about $200 \mathrm{~g}$ 's). By elevating the target $0.5 \mathrm{~mm} 10 \mathrm{msec}$ prior to the shot and retracting the support pedestal, the target velocity at shot time is only $0.01 \mathrm{~m} / \mathrm{sec}$. The allowable timing error for $5 \mu \mathrm{m}$ travel is an enormous $50 \mu \mathrm{sec}$. This inertial suspension scheme offers the possibility of being retrofitted to the baseline design. Both free-fall schemes require interlocks to assure target positioning prior to firing.

\subsubsection{Summary}

Cryogenic targets can be readily transported from layering and characterization to the shot point in such a way that target integrity is retained. Temperatures can be controlled within $\pm 1 \mathrm{~K}$ and forces held to well under $0.25 \mathrm{~g}$ 's. Target exposure to $300 \mathrm{~K}$ chamber radiation can be limited to 20-30 msec, which keeps target temperature within specs.

To accomplish this, we have selected a baseline design that uses a vertically-articulating injection stalk cooled by slow-flowing helium gas. An alternate is a conveyor system, similarly cooled, which reduces stalk travel and increases flexibility in placing of the layering station. We have adopted a dual shroud system, one for gas sealing and one for radiation blocking. The first, attached to the top of the chamber, retracts slowly. This eliminates vibrations and gives time for the helium exchange gas to vent and be partially pumped. The second, attached to an independent mounting system, retracts in 20-30 msec.

Two free-fall injection concepts are backup approaches: one through a cryogenic tube on the top of the chamber, and the other using the fast radiation shroud as the target support. In both cases, the target supports are grounded. The first system completely eliminates the need for shrouding, but introduces positioning uncertainties. The second system may be more precise, but does require the complex shrouding of the baseline design. An advantage of the second system is the ability to achieve it through retrofitting of the baseline. 


\section{SUMMARY}

We have proposed a cryogenic target insertion system comprising nine modules: Target Support, Fill Station(s), Transport to Storage, Cryogenic Storage, Transport to Layering, Layering, Characterization, Transport to Insertion, and Insertion. Of these the techniques chosen for the Target Support, Fill Station(s), Transport to Storage, Cryogenic Storage, Transport to Layering, and Insertion modules are hased on proven techniques. We are confident that those modules can be successfully designed and engineered to meet UR/LLE's requirements.

The techniques chosen for the Layering and Characterization modules are not sufficiently developed. The ICF national laboratories have the responsibility for specifying the layering and characterization techniques and to demonstrate that they are suitable for Omega Upgrade targets. Active research programs are currently underway at LLNL and LANL to develop the techniques.

Table II is a summary of the design requirements and how each module in our proposed conceptual design meets them.

\section{Requirement}

Target Support

4.3.1. thermally and mechanically stable

4.3.2. not interfere with laser or diagnostics

Cryogenic Fill Station

4.4.1. fill empty targets $>1000 \mathrm{~atm}$.

4.4.2. cool filled targets to below triple point

4.4.3. tritium inventory $<1$ gram of tritium six months DT supply for targets.

4.4.4. not release tritium

4.4.5. backup system for tritium recovery.

4.4.6. power failure protection

\section{Cryogenic Storage}

4.5.1 withstand loss of refrigerant w/o release of DT 4.5.2. hold at least six filled targets

Transport

4.6.1. $\Delta \mathrm{T}< \pm 0.2 \mathrm{~K}$

4.6.2. not allow release of DT to the environment

\section{Layering}

Characterization

Target Insertion

4.9.1. compatible with OMEGA laser and diagnostics

4.9.2. interlock to prevent damage to laser or optics

4.9.3. $\pm 5 \mathrm{~mm}$ positioning travel

4.9.4. $\pm 5 \mu \mathrm{m}$ positioning accuracy

4.9.5. maintain target to $\pm 5 \mu \mathrm{m}$ prior to shot

4.9.6. gas pressure $<10^{4}$ torr at the tank center

4.9.7. cycle targets in less than two hours

Safety and Environmental Requirements

4.10.1. meet local, state and federal ES\&H Reqs.

4.10.2. tritium inventory < 1 gram

4.10.3. zero release of tritium to environment.
Table II

Met? Comments

$\varphi$

$\checkmark$ designed to 1,500 aim.

$\checkmark$

$\checkmark$ Booster pump could be eliminated if inventory increased

$\checkmark$ Triple containment: permeation cell, fill cryostat, glove box

$\checkmark$ Uses existing UR/LLE system

$\checkmark$ Uses existing UR/LLE system

Tritium contained within cryostat at $<1$ atm.

We believe this can be relaxed to $\pm 1 \mathrm{~K}$

$\checkmark$ Tritium contained within cryostat at $<1$ atm.

? To be determined

? To be determined

$\checkmark$ Optical interlock

$\checkmark \mathrm{X}-\mathrm{Y}-\mathrm{Z}$ positioners

$\checkmark$ Target examined through twc-axis view ports

$\checkmark$ Vibration from shroud is isolateo

$\checkmark$ Dual shroud allows gas to dissipate over longer time

? Not demonstrated, but believed possible

? URILE to determine this

$\checkmark$

$\checkmark \operatorname{se\theta } 4.4 .4 ; 4.5 .1 ; 4.6 .2$ 
We believe we can meet the schedule needed by LLE provided funding on a year-to-year hasis is appropriate for the work scheduled and the techniques for the two items of greatest technical uncertainty, layering and characterization, are identified and demonstrated to be suitable for Omega Upgrade cryogenic targets by December 1993. The failure of either of the assumptions will substantially delay the schedule. 


\section{APPENDIX A}

Steps and amounts of DT involved in high pressure fills.

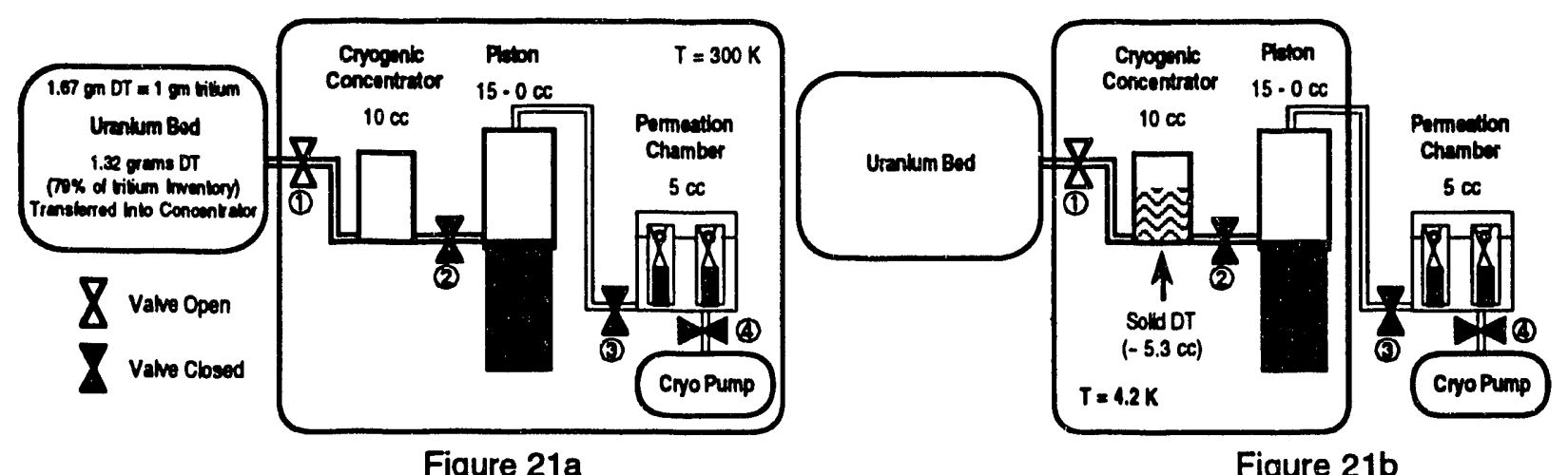

Figure 21a

Figure 21b

The first step in filling a target is to heat up the uranium bed to $450^{\circ} \mathrm{C}$, driving off the $\mathrm{D}_{2}$, DT and $T_{2}$ mixture which is the DT fill gas. 1.32 grams of the fill gas (79\% of the tritium inventory) is condensed (either as a liquid or solid) in a cryogenic concentrator that is cooled to near liquid helium temperatures (Figs. $21 \mathrm{a}$ and $\mathrm{b}$, above). In a pure $\mathrm{D}_{2}$ fill system, the uranium bed would be replaced by a bottle of $D_{2}$ gas.

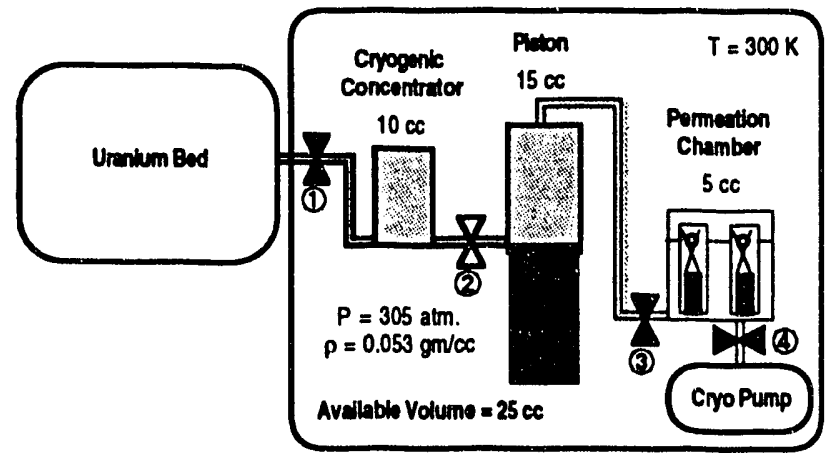

Figure 22a

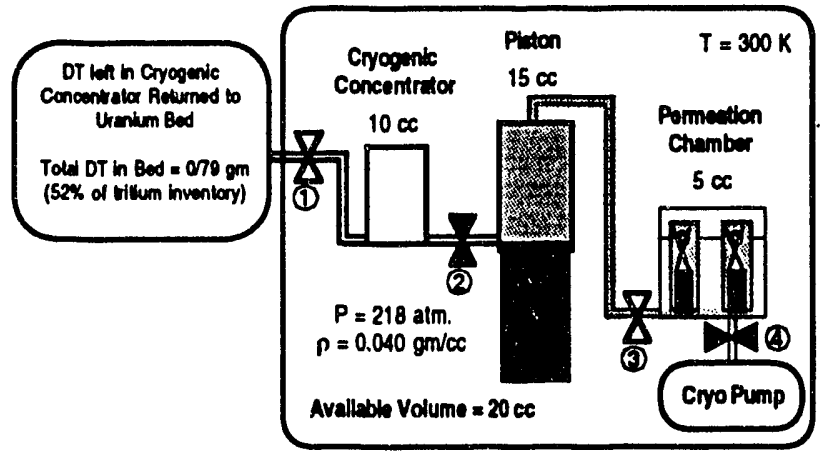

Figure 22b

Valve 1 is closed, valve 2 is opened and the temperature of the cryogenic concentrator is allowed to return to room temperature. The condensed DT vaporizes and, heing confined in a $25 \mathrm{cc}$ volume, rises to a pressure of 305 atmospheres (Fig. 22a). Valve 2 is closed and the DT remaining (0.44 grams) in the $10 \mathrm{cc}$ volume of the cryogenic concentrator is returned to the uranium hed. Opening valve 3 lets the fill gas into the $5 \mathrm{cc}$ volume of the permeation chamber, further decreasing the pressure to 218 atmospheres (Fig. 22b). 


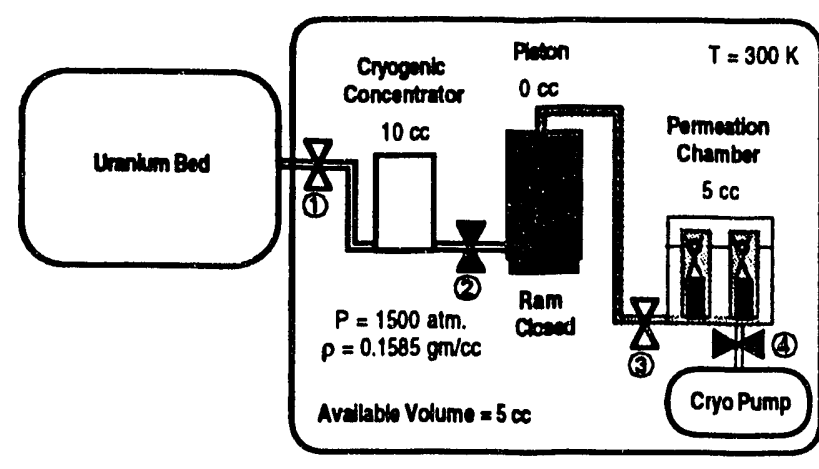

Figure 23a

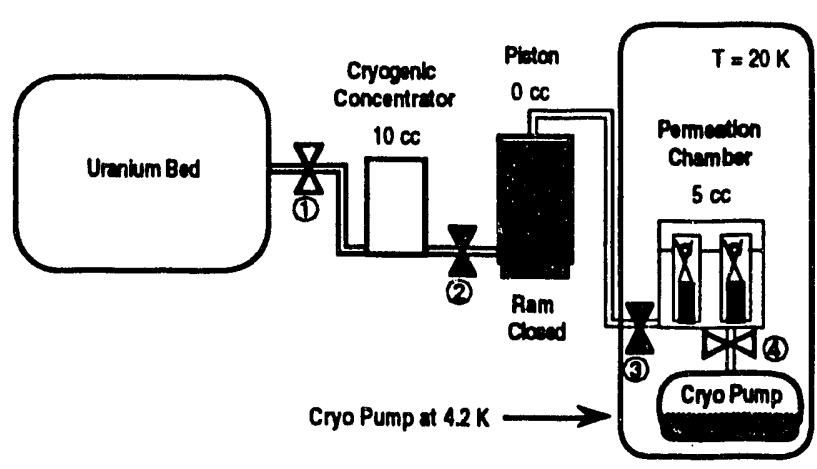

Figure 23b

The piston is then closed decreasing its volume to $0 \mathrm{cc}$ (total volume decreasing from $20 \mathrm{cc}$ to $5 \mathrm{cc}$ ) and increasing the pressure in the permeation cell to 1500 atmospheres (Fig. 23a). Once the empty shells have been filled, valve 3 is closed and the permeation chamber temperature is lowered to 20 25 K liquefying the fill gas and dropping the pressure below one atmosphere. Valve 4 is then opened and the DT remaining in the permeation chamber is condensed at $4.2 \mathrm{~K}$ in the cryopump (Fig. 23b).

After the target(s) have been removed, the cryopump is connected to the uranium bed (not shown) and warmed to room temperature. This returns all remaining DT to the uranium bed. 


\section{APPENDIX B}

\section{Detailed Description of Transport to Storage and Cryogenic Storage Modules}

The fill station cryostat is a variable temperature, closed cycle cryostat. The features of note are illustrated in Fig. 24. A gate valve and ISO-KF flange provide for transport tube \#1 and the cryogenic extension wrench to be inserted into and withdrawn from the cryostat through a vacuum lock. This prevents any possibility of gases leaking into or out of the cryostat. Equipment, components, and eventually the targets in the fill station cryostat are cooled via helium exchange gas held at pressures of about one torr. The thermal conductivity of helium is independent of pressure below one torr. In the event of a complete loss cooling, the helium exchange gas pressure will not exceed atmospheric pressure. If the free volume of the cryostat is at least $4 \mathrm{cc}$, the pressure of the DT fuel in six targets and the exchange gas will not exceed atmospheric pressure. A quantity of DT fuel at triple point liquid density, in a shell with inner and outer radii of 600 and $700 \mu \mathrm{m}$, respectively, will fill a volume of $0.6 \mathrm{cc}$ at $20^{\circ} \mathrm{C}$ and one atmosphere pressure.

The fill cryostat has two cryogenic chambers, upper and lower, separated by a plate. An axially centered hole in this plate allows vertical access into the lower chamber. This hole can be opened and closed with the movable baffle. The permeation cell is located in the lower chamber. With the baffle closed, initially warm equipment such as the cryogenic extension wrench can be slowly inserted into the upper chamber and cooled to cryogenic temperatures without the possibility of a heat pulse traveling through the helium exchange gas to the permeation cell located in the lower chamber. Once inserted equipment has cooled to cryogenic temperatures, the baffle is opened to permit access to the permeation cell.

The upper chamber houses a movable tray and the cryo-shroud remover. The tray can swing into and out of the center of the cryostat. The tray provides a place to put and then remove out of the way the permeation cell's cap and sub-cap. This speeds up operation, since the tool used to remove the cap and sub-cap, the cryogenic extension wrench, only has to be inserted and withdrawn from the cryostat once instead of twice. The cryo-shroud remover is used to attach and detach the cryo-shrouds from the bottom of cold transport tube \#1. Two concentric shafts and a transmission within the cryo-shroud remover allow it to swing a socket into the center of the cryostat and then independently rotate that socket.

The permeation cell cap and sub-cap have a peg on their top surface of the same diameter. This allows both to be picked up by the same tool. The cap and the upper permeation cell have an outer hexagonal cross-section. This permits the cap to be installed and removed with no net torque using two wrenches, one to apply a torque, the other to apply a counter-torque. 
A $4.2 \mathrm{~K}$ cryopump is located in the fill station cryostat. It is attached to the permeation cell with a short length of tubing. When the permeation cell is at the triple point of the DT fill gas, the cryopump is used to remove the DT external to the targets in the permeation cell. Windows are provided on the side of the cryostat at the level of the top of the permeation cell. These allow equipment to be centered directly over the center of the permeation cell. This is particularly useful when picking of the permeation cell cap, sub-cap, and the target rack.

Once the targets are filled, condensed, the external DT removed, and cold helium exchange gas is introduced into the permeation cell, the cap and sub-cap of the permeation cell must be removed. This is done with the cryogenic extension wrench. This consists of three concentric devices and a vacuum lock. All the devices are sealed at the top with sliding o-rings that allow independent rotation and translation of the devices with respect to each other. The wrench set is shown attached to the top of the fill station cryostat in Fig. 25. The outer two devices are stainless steel tubes with socket wrenches at their tips. These are used to unscrew the cap of the permeation cell with no net torque by applying a torque to the cap and a counter-torque to the cell. This reduces torsional strain on the neck of the fill station cryostat. The inner most device of the wrench set is a mechanical pickup tool. It consists of a shaft within a tuhe, both stainless steel. At the end of the shaft, protruding from the tube are two wedge shaped, sprung pinchers. Pushing down on the shaft allows the pinchers to spring open. Pulling up on the shaft forces the pinchers closed. Lips on the pinchers and the pegs on the cap and sub-cap prevent the possibility of the pickup tool dropping the cap or sub-cap once the pinchers are closed onto their peg.

The cryogenic extension wrench is operated as follows. The wrench is attached to the fill station cryostat through its vacuum lock (Fig. 25). The cryo-shroud remover and the tray are swung to the sides of the cryostat and the baffle swung to close the hole separating the upper and lower chamber of the cryostat. The vacuum lock is evacuated and then its gate valves opened. The wrench is slowly pushed into the exchange gas of the upper chamber. The rate of entry into upper chamber is controlled to be slow enough to prevent heating of the permeation cell. Several temperature sensors (not shown in figures) at various elevations in the cryostat are used to monitor the cryostat's temperature profile.

Once the wrench has cooled to cryogenic temperatures in the upper chamber, the baffle is swung out of the way and the wrench lowered to engage the permeation cell and its cap. The wrench is used to unscrew the cap (Fig. 26). Once the cap has been unscrewed the mechanical pick up tool in the wrench grasps the peg on the top of the cap. The cap is pulled up to a position slightly above the tray in the upper chamber. The tray in swung to the center of the cryostat, underneath the cap being held by the wrench (Fig. 27). The cap is deposited onto the tray and the tray swung out of the way to the side of the cryostat. 
The wrench is again lowered into the lower chamber. This time it picks up the sub-cap and the entire wrench is withdrawn into the vacuum lock. The lock's gate valves are closed and the wrench detached from the fill station cryostat. The baffle is moved to the closed position. The cryo-shroud remover is swung to the center of the cryostat in preparation for insertion of cold transport tube \#1.

Transport tube \#1 is used to pick up the target rack containing the filled targets from the fill station cryostat and drop it off in the cold storage cryostat, while at all times maintaining the temperature of the targets below their fuel's triple point. Transport tube \#1 is a cryostat especially designed so that its bottom section can be removed and attached while inside another cryostat. With its bottom removed, transport tube \#1 can pick up the target rack. The bottom section of the transport tube can then be re-attached, surrounding the target rack with an independent cryogenic environment. With its bottom section attached, the transport tube, with targets in tow, can be withdrawn and detached from the cryostat it was in, transported to another cryostat, and inserted into that cryostat. In the new cryostat, transport tube \#1 can again have its bottom section removed. Then the target rack can be dropped off at the bottom the new cryostat.

The basic design of transport tube \#1 is shown in Fig. 28. The lower section has a vacuum lock that surrounds, transport tube \#1's cryo-shroud. The upper section of transport tube \#1 consists of, from the outside in, a vacuum space, a liquid nitrogen space, a vacuum space, an annular closed cycle cryostat, a helium exchange gas space for cooling the targets, and the tube and shaft of a mechanical pick up tool of the type used in the cryogenic extension wrench. The outer vacuum space and the liquid nitrogen space are permanently sealed at the bottom. The inner vacuum space and the exchange gas space are sealed by the removable cryo-shroud. A coarse Z-positioner on the vacuum lock and fine Z-positioners on the mechanical pickup tool permit the pick up tool to be positioned precisely in the fill station cryostat and the cold storage cryostat.

The cryo-shroud has two main parts. The outer can of the cryo-shroud is threaded onto the outer wall at the bottom of transport tube \#1 squeezing a seal. Low thermal conductivity spacers with a star cross-section are used to separate the cans of the cryo-shroud and the walls of the transport tube. Under most operating conditions, the bottom of the transport tube is at the temperature of liquid nitrogen, $77 \mathrm{~K}$. Conduction will cause the cryo-shroud outer can and its seal to be at this temperature. The transport tube must be inserted far enough into the vacuum lock to allow a thermal gradient to develop between the cryo-shroud outer can $(77 \mathrm{~K})$ and the portion of the transport tube outer wall above the vacuum lock (room temperature) that keeps the o-rings in the vacuum lock contacting the transport tuhe outer wall at a functional temperature. With an outer wall made of stainless steel of the size anticipated, the cryo-shroud top should be about $10 \mathrm{~cm}$ helow the o-rings. The inner can of the cryo-shroud slides and rotates inside the outer can on low thermal conductivity slides. 
A spring between the inner and outer cans maintains a constant force on the seal between the transport tube's cryostat and the inner can of the cryo-shroud, once the outer can of the cryoshroud has been attached to the transport tube. This constant force will be maintained even through temperature changes and should negate any deleterious effects of thermal contraction of the seal. The ability of the inner can to rotate with respect to the outer can means that the seal material and faces will not be rotated as the seal is tightened. If required, a more complex design for the cryoshroud can also provide a constant force and non-rotation to the outer can's seal. KEL-F should be a suitable seal material. Its elastic modulus increases by only a factor of 3.6 to 5.4, depending on grade, as the temperature decreases from room temperature to $10 \mathrm{~K}$. The key at the bottom of the cryo-shroud allows the cryo-shroud to be rotated by the cryo-shroud removers' socket located in the fill station cryostat and the cold storage cryostat.

A crude model of the transport tube is used to estimate the acceptable leak rate for helium gas through the seals of its cryo-shroud. The model consists of two concentric tubes one meter in length. The outer tube has radius $19.05 \mathrm{~mm}$ and temperature $77 \mathrm{~K}$. The inner tube has radius 12.7 $\mathrm{mm}$ and temperature $15 \mathrm{~K}$. A pressure gauge is attached to the outer tube. The space between the tubes is pumped on with a pump with a speed of 1 liter/sec. This is about five times the conductance of the annulus between the tubes. The heat flow between the two tubes due to conduction by low pressure (molecular flow) helium gas is calculated to be $9.3 \mathrm{~W} / \mathrm{Pa}$ of helium. Low pressure means that the mean free path of the helium gas is larger than the separation between the tubes. The accommodation coefficient (the inverse of the number of wall collisions needed to thermalize a gas molecule) was taken to be 0.5 .

Commercial closed cycle refrigerators are available with cooling powers of at least $28 \mathrm{~W}$ at $15 \mathrm{~K}$. For perspective, $4.2 \mathrm{~K}$ liquid helium is vaporized to a $15 \mathrm{~K}$ gas at a rate of $0.35 \mathrm{liter} / \mathrm{watt} \cdot \mathrm{hr}$. Assume that a one watt heat leak to the inner tube is acceptable. Then the allowable helium pressure between the tubes is $8.1 \times 10^{-4}$ torr. The system pumping speed times the allowable pressure gives the acceptable leak rate of the seals as $1.8 \times 10^{-4} \mathrm{~atm} \cdot \mathrm{cc} / \mathrm{sec}$. Note that since the pressure of the helium exchange gas to be used is about one torr, the pressure difference driving the leak will only be about one torr."

The operating procedure of the transfer tube \#1 to transport the target rack and targets cold from the fill station cryostat to the storage cryostat is as follows. After the permeation cell cap and subcap are removed from the permeation cell, the fill station cryostat's baffle is closed, and the fill station cryostat's cryo-shroud remover is swung into the center of the cryostat, the transport tube is attached to the fill station cryostat through the vacuum lock (Fig. 29). The liquid nitrogen space of the transport tube is pumped on until the liquid nitrogen freezes. The nitrogen will form a low thermal conductivity powder and eliminates convection that would be present in the liquid 
nitrogen. This allows the transport tube outer walls to cool more easily when the transport tube is inserted into the fill station cryostat.

The vacuum lock is evacuated and its gate valves opened. The transport tube is immediately lowered to an elevation within the upper chamber of the fill station cryostat where the temperature of the helium exchange gas is that of solid nitrogen, about $56 \mathrm{~K}$ when the solid's vapor pressure is 20 torr. The transport tube is then slowly lowered further into the upper chamber. The rate of lowering is kept slow enough to prevent the permeation cell from being warmed. Temperature sensors at various elevations in the fill station cryostat give its temperature profile.

When the cryo-shroud has reached cryogenic temperatures (less than the triple point of DT, 19.79 $\mathrm{K})$, the transport tube is further lowered until the cryo-shroud is inside the socket of the cryoshroud remover (Fig. 30). The region of cryogenic temperatures in the fill station cryostat extends to a height that is at least twice the length of the cryo-shroud above the base of the cryo-shroud socket. The transport tube is firmly held while the cryo-shroud remover socket unscrews the cryoshroud from the transport tube. The transport tube is then raised so that its mechanical pick up tool is just above the cryo-shroud that has been left behind in the cryo-shroud remover socket. Then the cryo-shroud left behind in the cryo-shroud remover is swung out of the way to the side of the fill station cryostat and the baffle opened.

The transport tube is lowered into the lower chamber of the fill station cryostat. The mechanical pick up tool is used to grasp the central shaft of the target peg (Fig. 31). The transport tube is raised to a position that brings the target rack to a height just above the top of the cryo-shroud, which is still located in the cryo-shroud remover. Figure 31, shows the transport tube as it is first beginning to raise the target rack. The cryo-shroud remover is swung to the center of the fill station cryostat to bring the cryo-shroud directly underneath the transport tube. The transport tube is lowered to engage the cryo-shroud. The cryo-shroud remover socket is rotated to screw the cryo-shroud back onto the transport tube (Fig. 32).

The helium exchange gas in the transport tube inner vacuum space is evacuated through a port at the top of the transport tube. The helium exchange gas in the transport tube exchange gas space, where the target rack is currently located is not pumped out. The helium exchange gas inside the fill station cryostat, including the vacuum locks is evacuated. The liquid nitrogen space pumping is stopped. This allows the solid nitrogen to liquefy. The transport tube is then raised into its vacuum lock and the gate valves closed. The transport tube is detached from the fill station cryostat at the vacuum lock (Fig. 33). Transport tube \#1 is then transported to a position where it can be attached to the storage cryostat. 
The storage cryustat is very similar to the fill station cryostat. The storage cryostat replaces the permeation cell with a target rack receptacle mounted on an X-Y translation stage. The movable tray and the $4 \mathrm{~K}$ cryopump are omitted. The storage cryostat is also separated by a plate into two cryogenic chambers, upper and :ower. A movabl jaffle, identical to the one used in the fill station cryostat, covers and uncovers a central hole in the plate that provides for vertical access to the lower chamber from the upper chamber. The $X-Y$ stage is in the lower chamber. Windows are placed in the sides of the storage cryostat at the height that targets will occupy. This allows observation of the X-Y stage positioning of a target directly under the mechanical pick up tool of a cold transport tube. The upper chamber contains a cryo-shroud remover identical to the one used in the fill station cryostat.

Transport tube \#1 is inserted into the storage crycstat in the same manner as it is inserted into the fill station cryostat. Figure 34 shows transf .rt tube \#1 dropping off the target rack into the receptacle at the bottom of the storage cryostat. After the target rack is dropped off in the receptacle, the transport tube's cryo-shrour is re-attached. Then the transport tube is withdrawn into its vacuum lock in the same procedure that was used with the fill station cryostat, but in this case the helium exchange in the storage cryostat is not pumped out. Once the gate valves of the vacuum locks are shut, the vacilim locks are evacuated. This delayed evacuation causes a thermal shock to transport tube \#1, but since it no longer contains targets, this is not a problem. This procedure is required to maintain exchange gas cooling of the targets in the storage cryostat. 


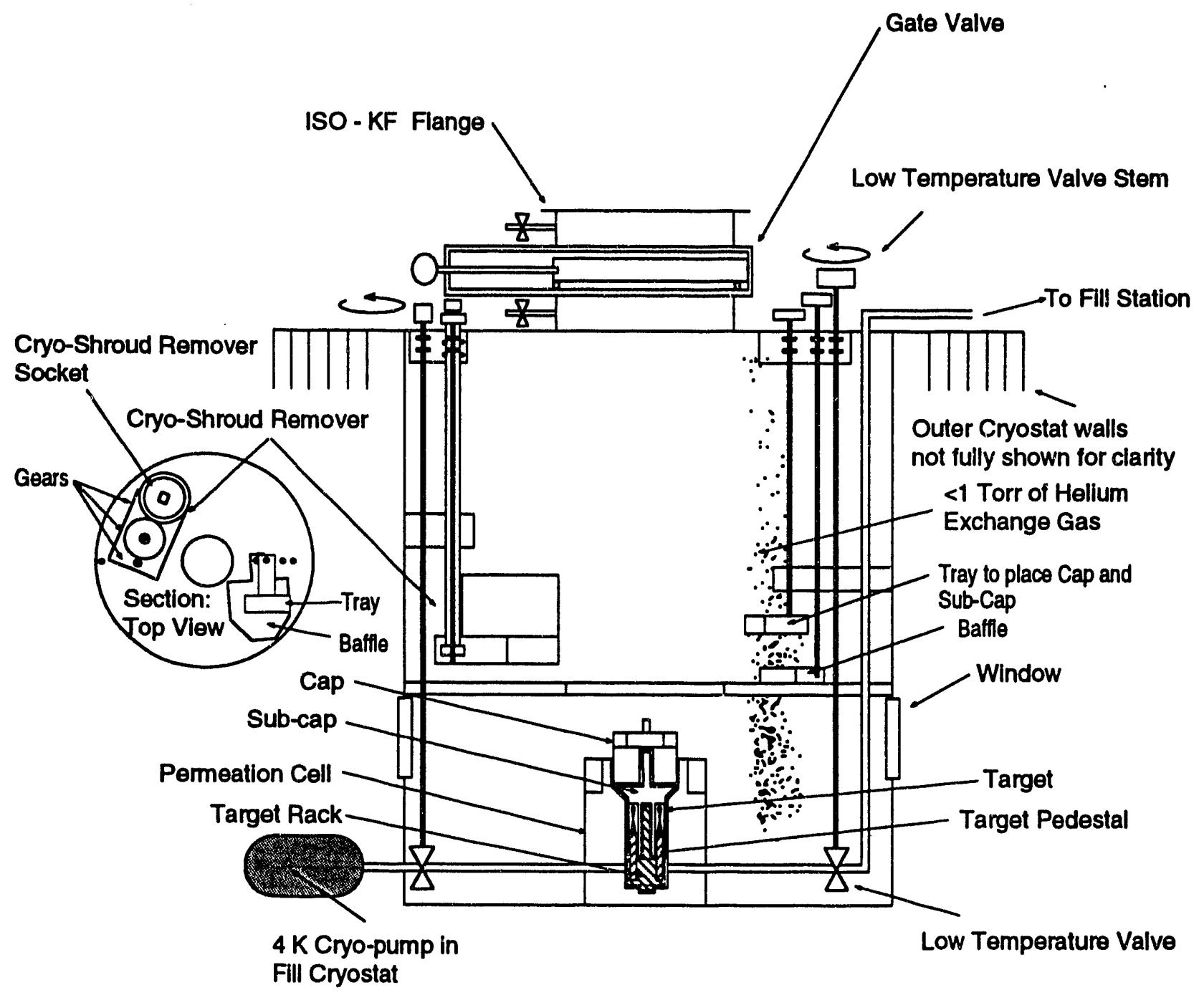

Figure 24: The fill station cryostat has filled targets sealed inside the cell. 


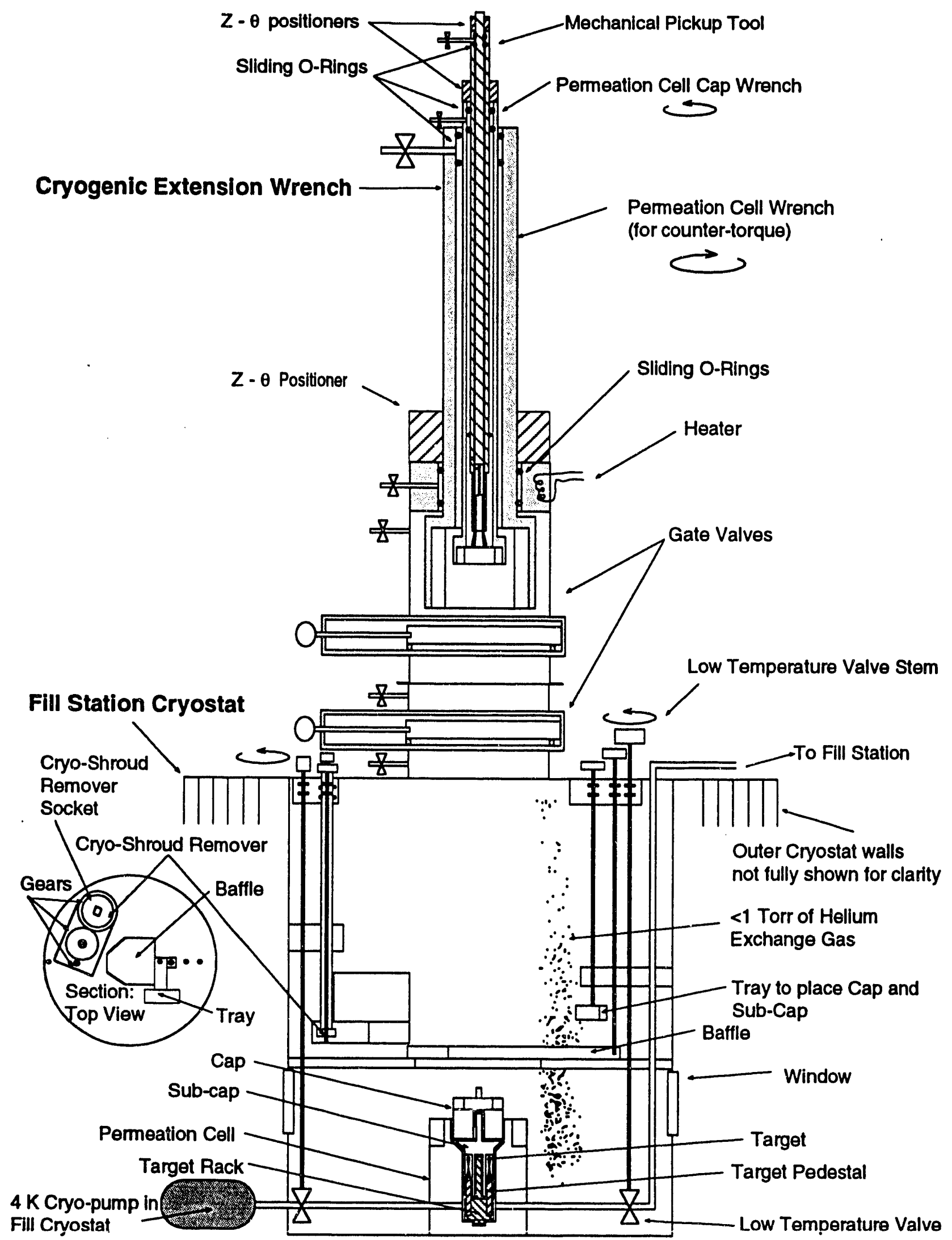

Figure 25: The cryogenic extension wrench is mated to the fill station cryostat. 


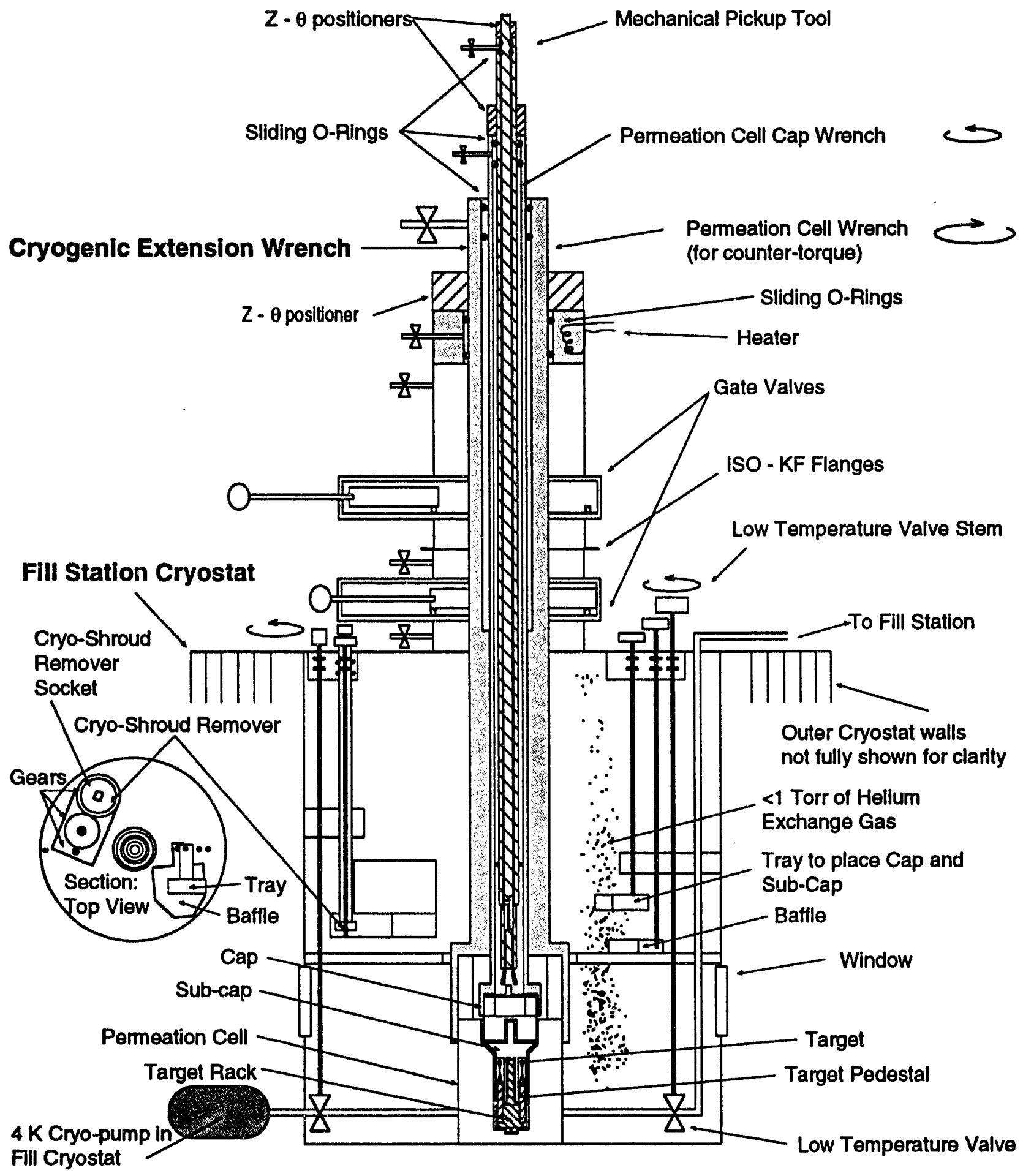

Figure 26: Torque and counter-torque are applied with the onjogenic extension urrench to unseal the permeation cell. 


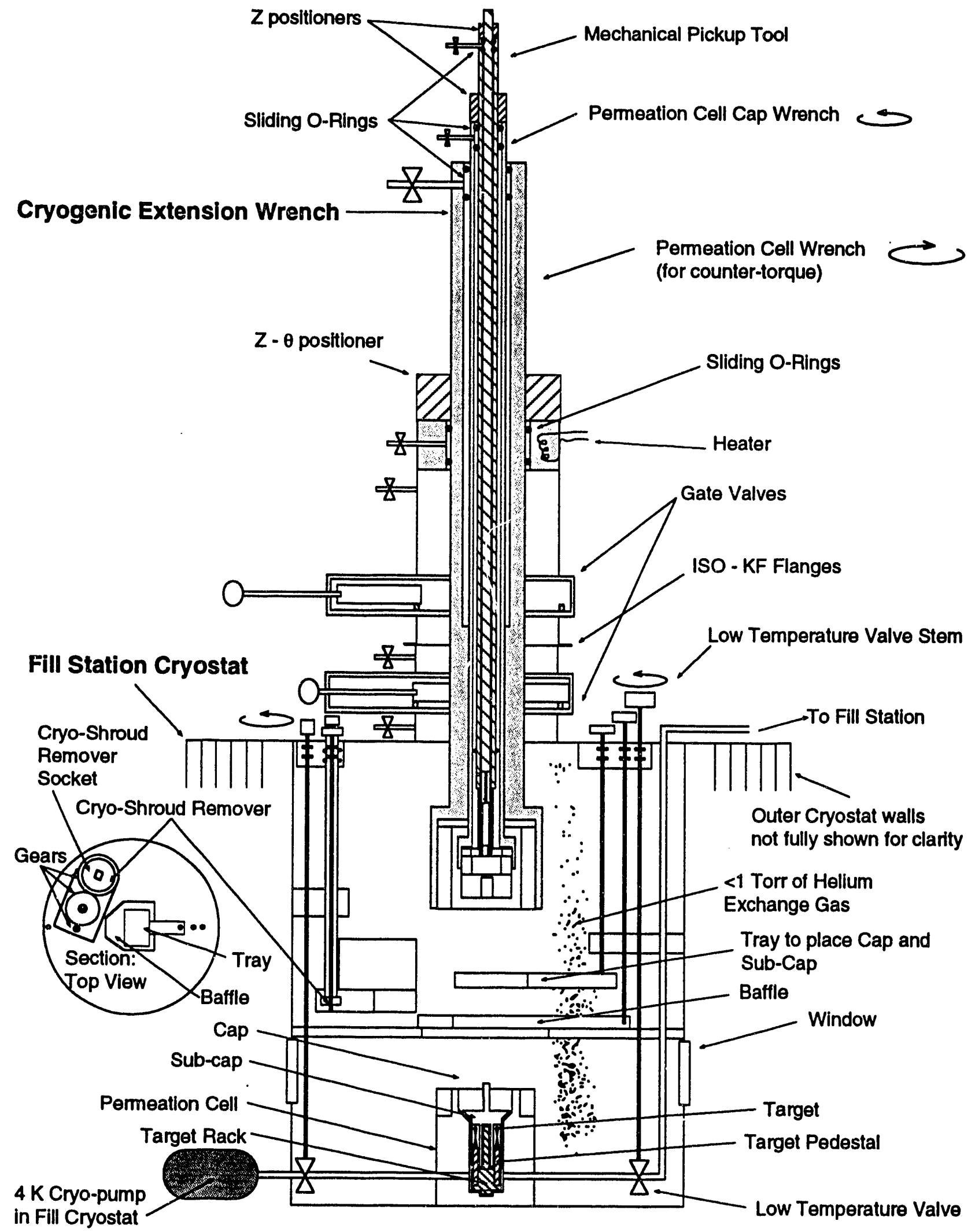

Figure 27: The cryagenic extension wionch has picked up the permeation ceil cap and is about to place it on the tray. 


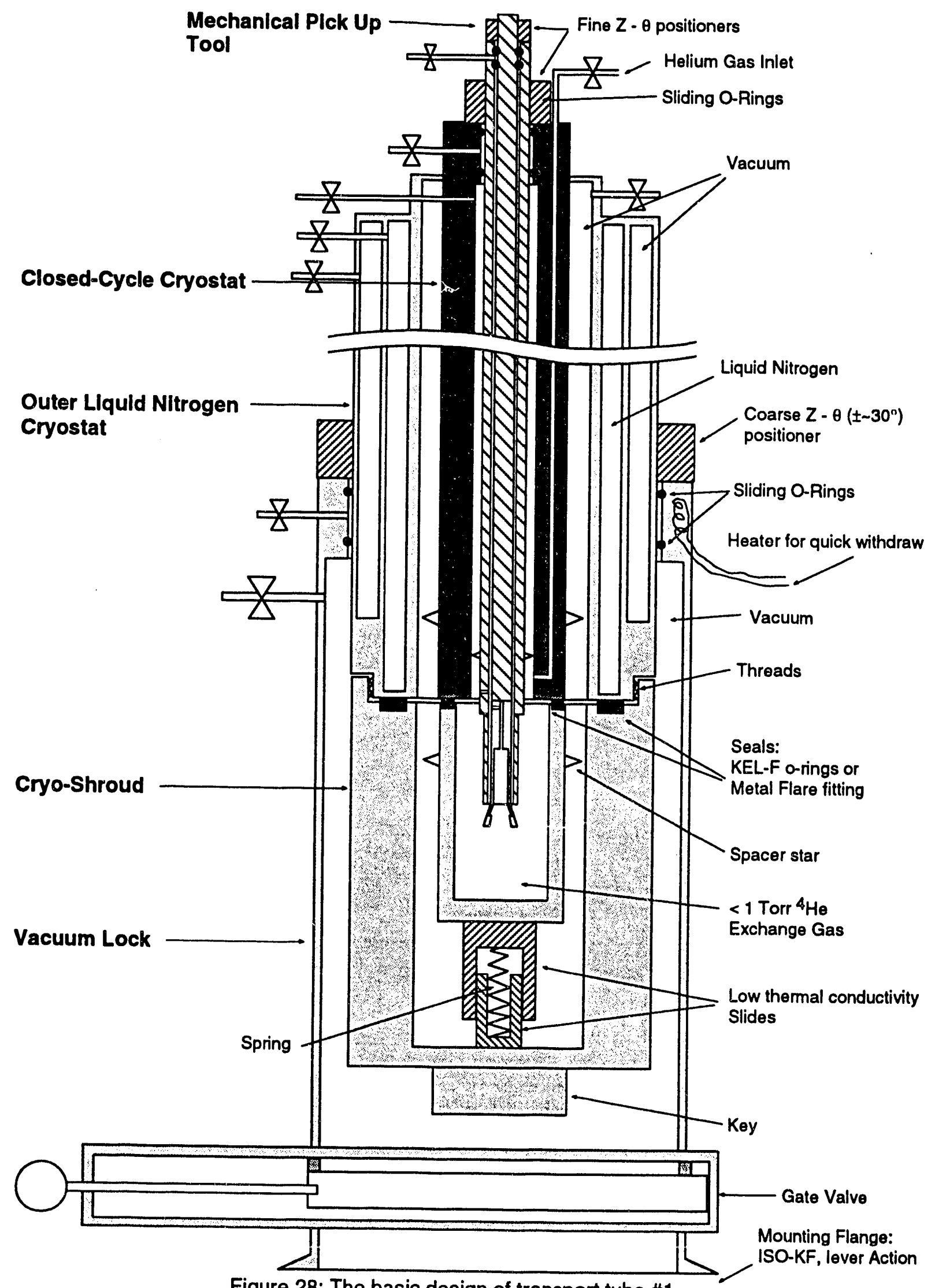

Figure 28: The basic design of transport tube \#1. 


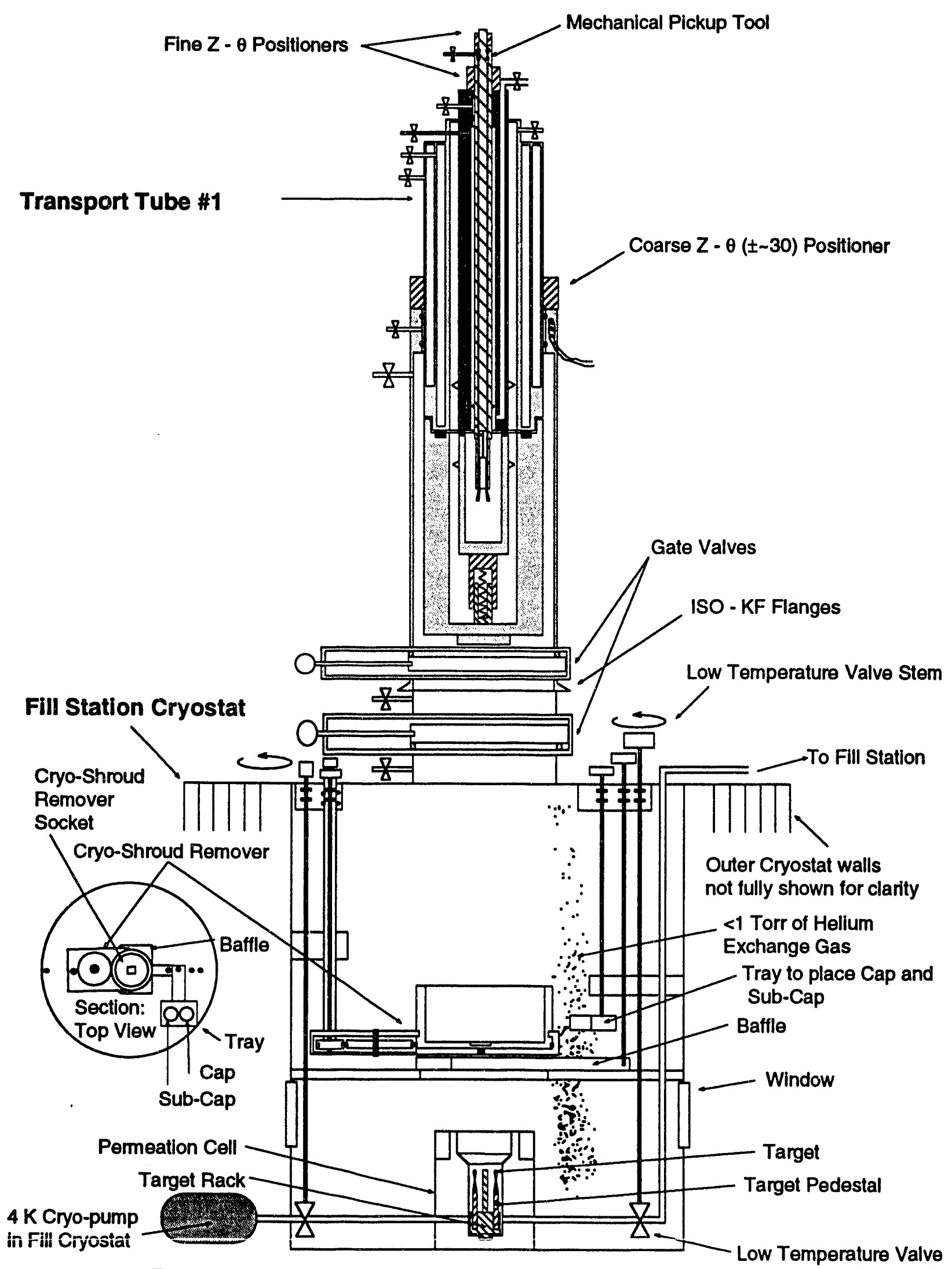

Figure 29: Transport tube \#1 is mated with the fill station cryostat. 


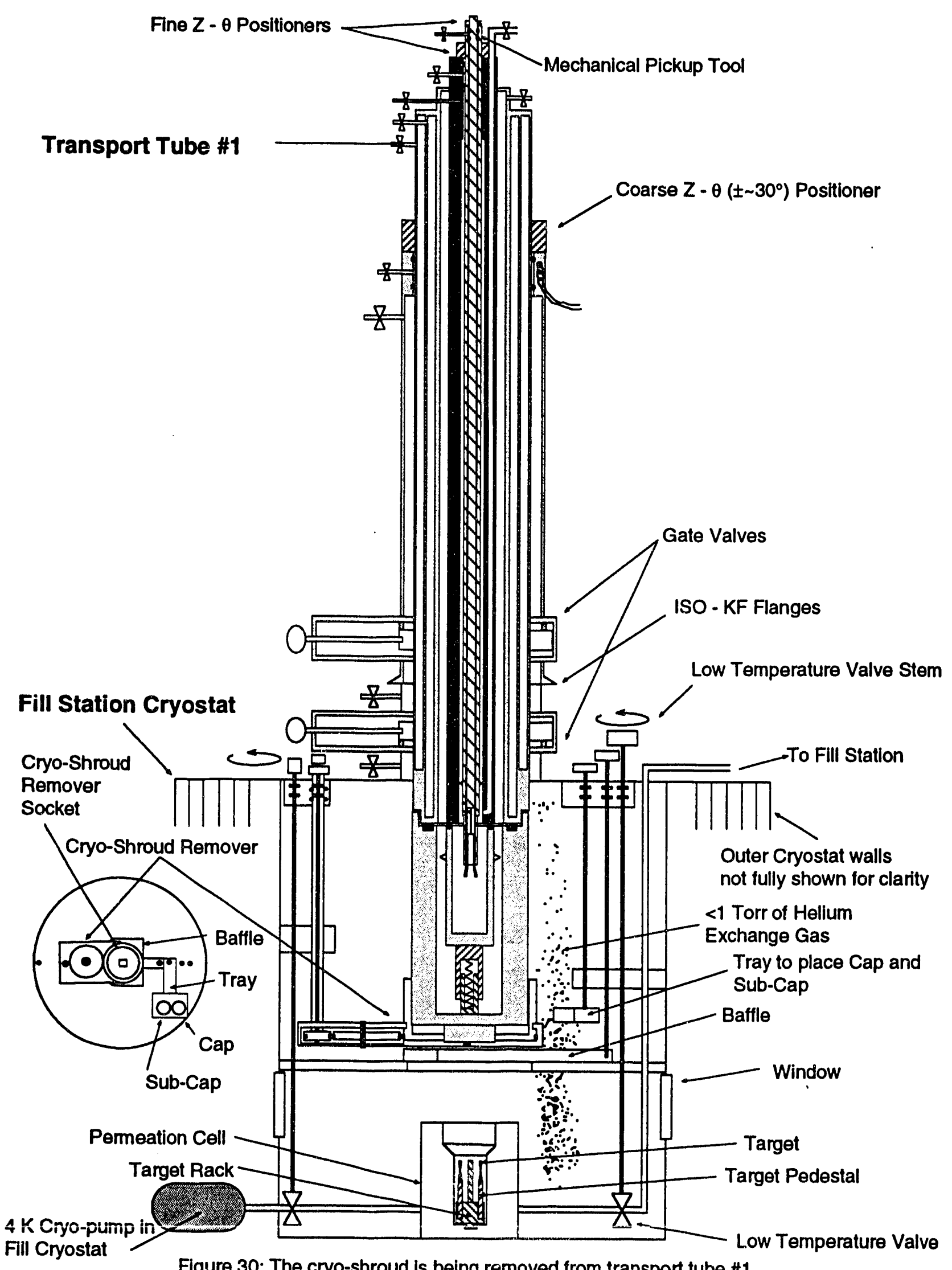

Figure 30: The cryo-shroud is being removed from transport tube \#1. 


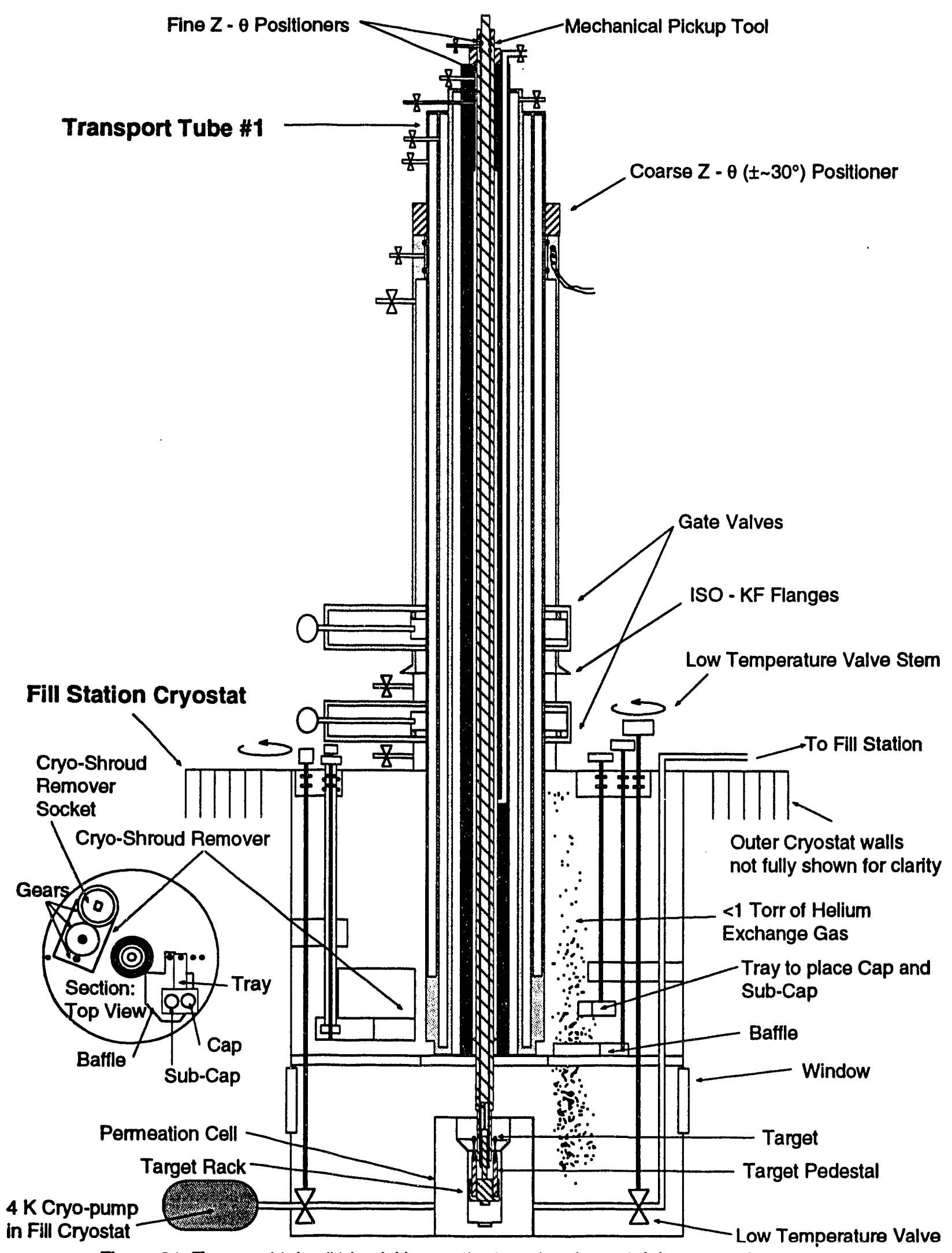

Figure 31: Transport tube \#1 is picking up the target rack, containing up to six targets, from the fill station cryostat. 


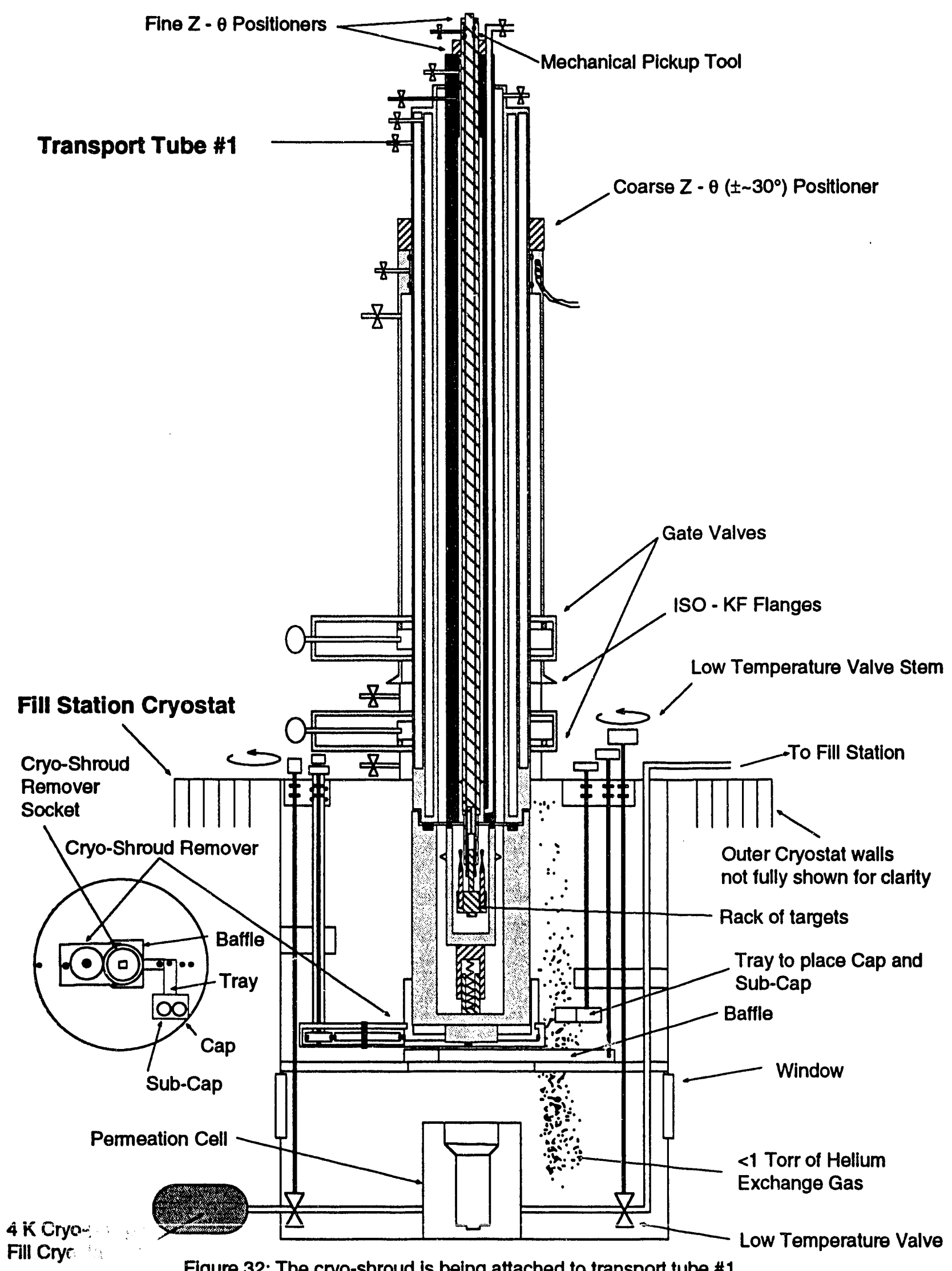

Figure 32: The cryo-shroud is being attached to transport tube \#1. 


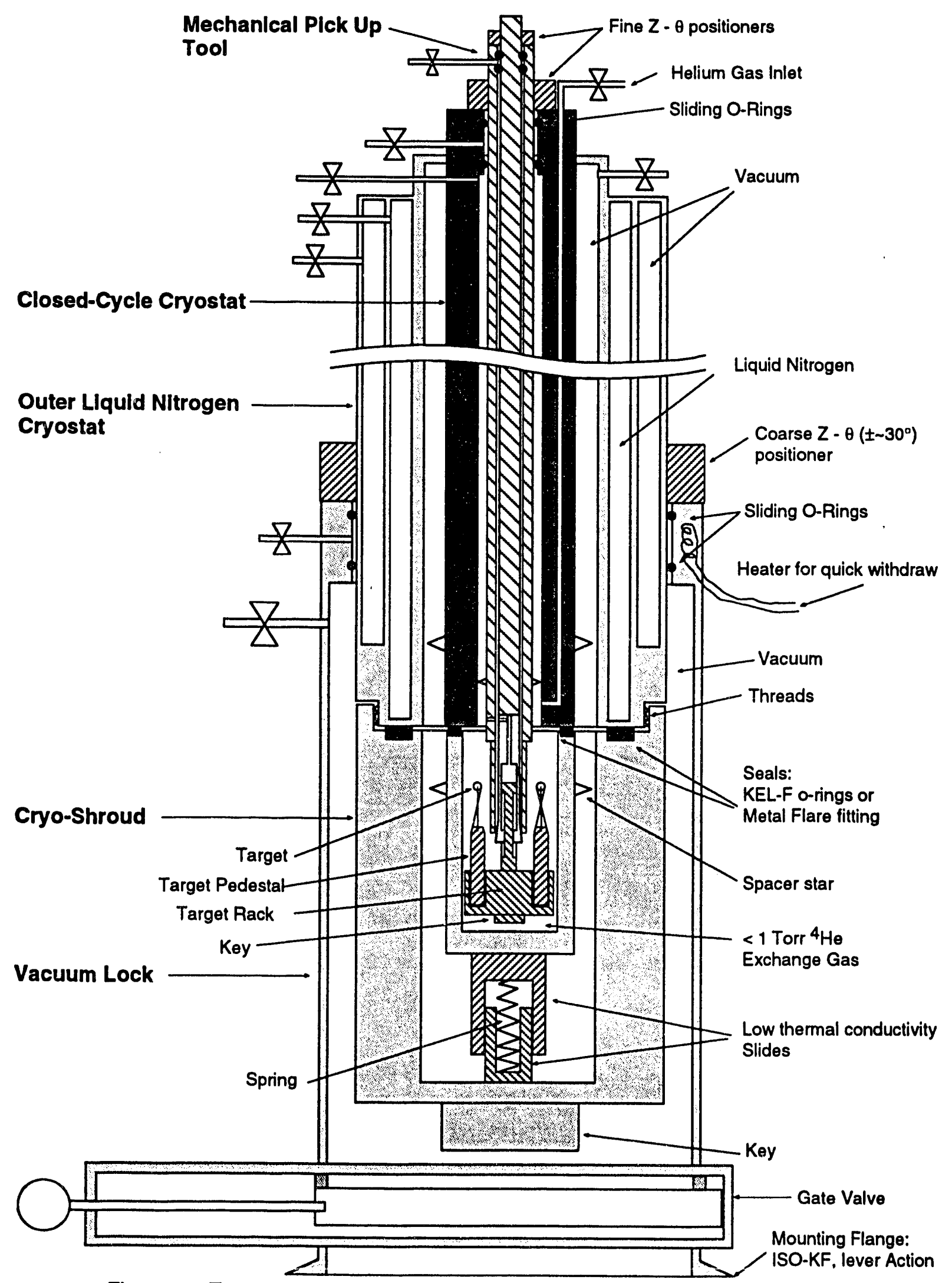

Figure 33: Transport tube \#1 is detached from the fill station cryostat and is transporting the target rack and targets to the cold storage cryostat. 


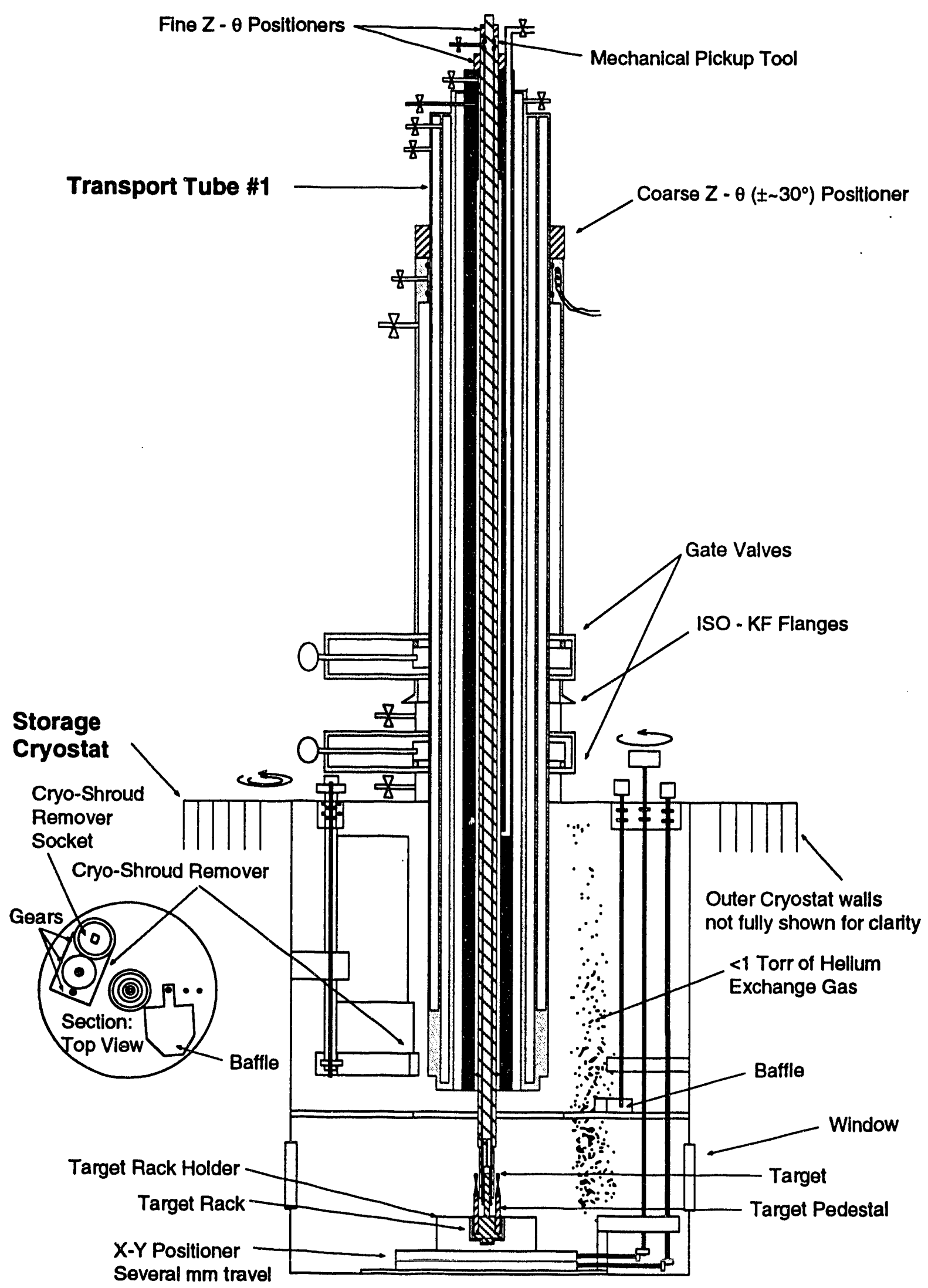

Figure 34: The rack of targets is being placed into the bottom of the storage cryostat by transport tube \#1. 


\section{Appendix C}

\section{Detailed Description of T'ransport to the Layering Module}

The cold storage cryostat is a variable temperature, closed cycle cryostat that moves targets from the storage cryostat to the layering apparatus (Fig. 35). The cold storage cryostat with transport tube \#2 attached is illustrated in Fig. 36. A gate valve and ISO-KF flange provide for transport tube \#1 and transport tube \#2 to be inserted into and withdrawn from the cryostat through a vacuum lock. This prevents any possibility of gases leaking into or out of the cryostat. Equipment, components, and eventually the targets in the cold storage cryostat are cooled via helium exchange gas held at pressures of about one torr. The thermal conductivity of helium is independent of pressure above one torr. In the event of a complete loss cooling, the helium exchange gas pressure will not exceed atmospheric pressure. If the free volume of the cryostat is at least $4 \mathrm{cc}$, the pressure of the DT fuel in six targets and the exchange gas will not exceed atmospheric pressure. A quantity of DT fuel at triple point liquid density, in a shell with inner and outer radii of 600 and $700 \mu \mathrm{m}$, respectively, will fill a volume of $0.6 \mathrm{cc}$ at $20^{\circ} \mathrm{C}$ and one atmosphere pressure.

The cold storage cryostat has two cryogenic chambers, upper and lower, separated by a plate. An axially centered hole in this plate allows vertical access into the lower chamber. This hole can be opened and closed with the movable baffle. The target rack receptacle mounted on an X-Y translation stage is located in the lower chamber. With the baftle closed, initially warm equipment such as the cold transport tubes can be slowly inserted into the upper chamber and cooled to cryogenic temperatures without the possibility of a heat pulse traveling through the helium exchange gas to the target rack located in the lower chamber. Once inserted equipment has cooled to cryogenic temperatures, the haffle is opened to permit access to the targets.

The upper chamber houses the cryo-shroud remover. The cryo-shroud remover is used to attach and detach the cryo-shrouds from the bottom of cold transport tubes \#1 and \#2. Two concentric shafts and a transmission within the cryo-shroud remover allow it to swing a socket into the center of the cryostat and then independently rotate that socket. Windows are provided on the side of the cold storage cryostat at the level that the targets in the X-Y stage will occupy. These, together with the $X-Y$ translation stage, allow a target to be centered directly under the center of the mechanical pick up tool of cold transport tuhe \#2. This is particularly useful when picking up a single target.

Transport tube \#2 is used to pick up a single target from the cold storage cryostat and drop it off in the layering cryostat, while at all times maintaining the temperature of the target below its fuel's triple point. Transport tube \#2 is of identical design to that of cold transport tube \#1 except for the shape of the pinchers in the mechanical pick up tool. Transnort tube \#2 is a cryostat especially designed so that its bottom section can he removed and attached while inside another cryostat (Fig. 37). 
With its bottom removed, transport tube \#2 can pick up a single mounted target. The bottom section of transport tube \#2 can then be re-attached surrounding the target with an independent cryogenic environment. With its bottom section attached, transport tube \#2, with target in tow, can be withdrawn and detached from the cryostat it was in, transported to another cryostat, and inserted into that cryostat. In the new cryostat, tube $\# 2$ can again have its bottom section removed. Then the target rack can be dropped off at the bottom the new cryostat. The basic design of transport tube \#2 is shown in Fig. 37.

The lower section has a vacuum lock that surrounds, tube \#2's cryo-shroud. The upper section of tube \#2 consists of, from the outside in, a vacuum space, a liquid nitrogen space, a vacuum space, an annular closed cycle cryostat, a helium exchange gas space for cooling the targets, and the tube and shaft of a mechanical pick up tool. The mechanical pickup tool consists of a shaft within a tube, both stainless steel. At the end of the shaft, protruding from the tube are two wedge shaped, sprung pinchers. Pushing down on the shaft allows the pinchers to spring open. Pulling up on the shaft forces the pinchers closed. Above their bottom tips, the pinchers are sufficiently hollowed out to allow the pinchers to be lowered down over the mounted target without touching the target. When the pinchers are closed in this position, the pinchers' tips grasp the target's pedestal mount with the target situated in the cavity formed by the hollowed out pinchers. The outer vacuum space and the liquid nitrogen space are permanently sealed at the bottom. The inner vacuum space and the exchange gas space are sealed by the removable cryo-shroud. Like in transport tube \#1, a coarse Zpositioner on the vacuum lock and fine Z-positioners on the mechanical pickup tool permit the pick up tool to be positioned precisely in the fill station cryostat and the cold storage cryostat.

The cryo-shroud has two main parts. The outer can of the cryo-shroud is threaded onto the outer wall at the bottom of transport tube \#2 squeezing a seal. Low thermal conductivity spacers with a star cross-section are used to separate the cans of the cryo-shroud and the walls of transport tube \#2.Under most operating condition the bottom of transport tube \#2 is at the temperature of liquid nitrogen, $77 \mathrm{~K}$. Conduction will cause the cryo-shroud outer can and its seal to be at this temperature. Transport tuhe $\# 2$ must be inserted far enough into the vacuum lock to allow a thermal gradient to develop between the cryo-shroud outer can $(77 \mathrm{~K})$ and the portion of transport tube \#2 outer wall above the vacuum lock (room temperature) that keeps the o-rings in the vacuum lock contacting transport tube \#2 outer wall at a functional temperature. With an outer wall made of stainless steel of the size anticipated, the cryo-shroud top should he about $10 \mathrm{~cm}$ below the o-rings. The inner can of the cryo-shroud slides and rotates inside the outer can on low thermal conductivity slides.

A spring between the inner and outer cans maintains a constant force on the seal between transport tube \#2's cryostat and the inner can of the cryo-shroud, once the outer can of the cryo-shroud has 
been attached to the transport tube \#2. This constant force will be maintained even through temperature changes and should negate any deleterious effects of thermal contraction of the seal. The ability of the inner can to rotate with respect to the outer can means that the seal material and faces will not be rotated as the seal is tightened. If required, a more complex design for the cryoshroud can also provide a constant force and non-rotation to the outer can seal.

KEL-F should be a suitable seal material. Its elastic modulus increases by only a factor of 3.6 to 5.4 , depending on grade, as the temperature decreases from room temperature to $10 \mathrm{~K}$. The key at the bottom of the cryo-shroud allows the cryo-shroud to be rotated by the cryo-shroud removers' socket located in the cold storage cryostat and the layering cryostat.

A crude model of transport tube \#2 is used to estimate the acceptable leak rate for helium gas through the seals of the cryo-shroud. The model consists of two concentric tubes one meter in length. The outer tube has radius $19.05 \mathrm{~mm}$ and temperature $77 \mathrm{~K}$. The inner tube has radius 12.7 $\mathrm{mm}$ and temperature $15 \mathrm{~K}$. A pressure gauge is attached to the outer tube. The space between the tubes is pumped on with a pump with a spe $2 ;$ of 1 liter/sec. This is about five times the conductance of the annulus between the tubes. The heat flow between the two tubes due to conduction by low pressure (molecular flow) helium gas is calculated to be $9.3 \mathrm{~W} / \mathrm{Pa}$ of helium. Low pressure means that the mean free path of the helium gas is larger than the separation hetween the tubes. The accommodation co-efficient (the inverse of the number of wall collisions needed to thermalize a gas molecule) was taken to be 0.5 .

Commercial closed cycle refrigerators are available with cooling powers of at least $28 \mathrm{~W}$ at $15 \mathrm{~K}$. For perspective $4.2 \%$ lyuid helium is vaporized to a $15 \mathrm{~K}$ gas at a rate of $0.35 \mathrm{liter} / \mathrm{watt} \cdot \mathrm{hr}$. Assume that one watt heat leak to the inner tube is acceptable. Then the allowable helium pressure between the tubes is $8.1 \times 10^{-4}$ torr. The system pumping speed times the ai:. wable pressure gives the acceptable leak rate of the seals as $1.8 \times 10^{-4} \mathrm{~atm} \cdot \mathrm{cc} / \mathrm{sec}$. Note that since the pressure of the helium exchange gas to be used is about one torr, the pressure difference driving the leak will only be about one torr.

The operating procedure of the cold transport tube \#2 to transport a single target from the cold storage cryostat to the layering cryostat is as follows. With the storage cryostat's baffle closed, and the cold storage cryostat's cryo-shroud remover swung into the center of the cryostat, transport tube \#2 is attached to the storage cryostat through the vacuum lock. The liquid nitrogen space of transport tube \#2 is pumped on until the liquid nitrogen freezes. The nitrogen will form a low thermal conductivity powder and eliminates convection that would be present in the liquid nitrogen. This allows transport tube \#2's outer walls to cool more easily when tube \#2 is inserted into the storage cryostat. 
The vacuum lock is evacuated and its gate valves opened. Transport tube \#2 is immediately lowered to an elevation within the upper chamber of the cold storage cryostat where the temperature of the helium exchange gas is that of solid nitrogen, about $56 \mathrm{~K}$ when the solid's vapor pressure is 20 torr. Transport tube \#2 is then slowly lowered further into the upper chamber. The rate of lowering is kept slow enough to prevent the targets in the target rack in the lower chamber from being warmed. Temperature sensors a various elevations in the cold storage cryostat give its temperature profile.

When the cryo-shroud has reached cryogenic temperatures (less than the triple point of DT, 19.79 $\mathrm{K})$, transport tube \#2 is further lowered until the cryo-shroud is inside the socket of the cryoshroud remover, identical to the procedure used with cold transport tube \#1. The region of cryogenic temperatures in the cold storage cryostat extends to a height that is at least twice the length of the cryo-shroud above the base of the cryo-shroud socket. Transport tube \#2 is firmly held while the cryo-shroud remover socket unscrews the cryo-shroud from transport tube \#2. Transport tube \#2 is then raised so that its mechanical pick up tool is just above the cryo-shroud that has been left behind in the cryo-shroud remover socket. Then the cryo-shroud left behind in the cryo-shroud remover is swung out of the way to the side of the cold storage cryostat and the baffle opened.

Transport tube \#2 is lowered into the lower chamber of the cold storage cryostat. The X-Y translation stage positions a single mounted target directly underneath the mechanical pick up tool. The mechanical pick up tool is used to grasp the pedestal of the target's mount (Fig. 38). Transport tube \#2 is raised to a position that brings the target to a height just above the top of the cryoshroud, which is still located in the cryo-shroud remover. The cryo-shroud remover is swung to the center of the cold storage cryostat to bring the cryo-shroud directly underneath transport tube \#2. Transport tube \#2 is lowered to engage the cryo-shroud. The cryo-shroud remover socket is rotated to screw the cryo-shroud back onto transport tube \#2, identical to the procedure used with cold transport tube \#1.

The helium exchange gas in transport tube \#2's inner vacuum space is evacuated through a port at the top of transport tube \#2. The helium exchange gas in transport tube \#2 exchange gas space, where the target is currently located, is not pumped out. The helium exchange gas inside the cold storage cryostat, including the vacuum locks is evacuated. The liquid nitrogen space pumping is stopped. This allows the solid nitrogen to liquefy. Transport tube $\# 2$ is then quickly raised into its vacuum lock, the gate valves closed, and immediately cold helium exchange gas re-introduced to the cold storage cryostat. If the baffle provides a leak-tight seal, the lower chamber will not lose exchange gas and the previous procedure will not be time critical. Transport tube \#2 is detached from the storage cryostat at the vacuum lock (Fig. 39). Transport tube \#2 is then transported to a position where it can be attached to the layering cryostat. 
The layering cryostat has the same features as the storage cryostat that permit it to operate with cold transport tube \#2. The layering cryostat is also separated into two cryogenic chambers, upper and lower. A movable baffle, identical to the one used in the storage cryostat, opens and closes a central hole in the plate that provides for vertical access to the lower chamber from the upper chamber. Figure 40 shows transport tube \#2 attached to the layering cryostat. Figure 41 shows the horizontal plan of part of the upper chamber. Figs. 42 and 43 show the horizontal plan of the lower chamber, including possible positions for thermal gradient layering jets and three mutually perpendicular Mach-Zehnder interferometers for characterization. At the bottom of the lower chamber, directly underneath the port to which transport tube \#2 is attached, is a post with an inset for the target mount attached onto a Z-positioner. Windows are placed in the sides of the storage cryostat at the height of the top of the inset target post. These allow insertion of the target mount into the inset of the target post to be monitored.

The inset target post has a Z-positioner so that the target can be moved precisely to the position of the intersection of the three characterization interferometers. Figure 44 shows that the inset target post's radius must be less than $9.7 \mathrm{~mm}$ for the post not to shadow the $f 1.6$ optics of the interferometers. The space that must remain clear above the inset target post to permit the mechanical pick up tool of the cold transfer tube \#2 to insert a target into the post is a cylinder of 3 $\mathrm{mm}$ radius. Figure 45 shows that this constraint implies that an $\mathrm{fl} 1.6$ lens in the interferometers will have to be positioned at least $4.7 \mathrm{~mm}$ from the target. Also located in the lower chamber is a cryogenic robotic arm. It is used to transport a layered target directly to the tip of the insertion system's insertion pylon. The upper chamber contains a cryo-shroud remover identical to the one used in the storage cryostat.

Transport tube \#2 is inserted into the layering cryostat in the same manner as it is inserted into the storage cryostat. After the target is dropped off in the inset target post, transport tube \#2's cryoshroud is re-attached. Then transport tube \#2 is withdrawn into its vacuum lock in the same procedure that was used with the storage cryostat, but in this case the helium exchange in the layering cryostat is not pumped out. Once the gate valves of the vacuum locks are shut, the vacuum locks are evacuated. This delayed evacuation causes a thermal shock to transport tube \#2, but since it no longer contains a target, this is not a problem. This procedure maintains exchange gas cooling of the target in the layering cryostat. 


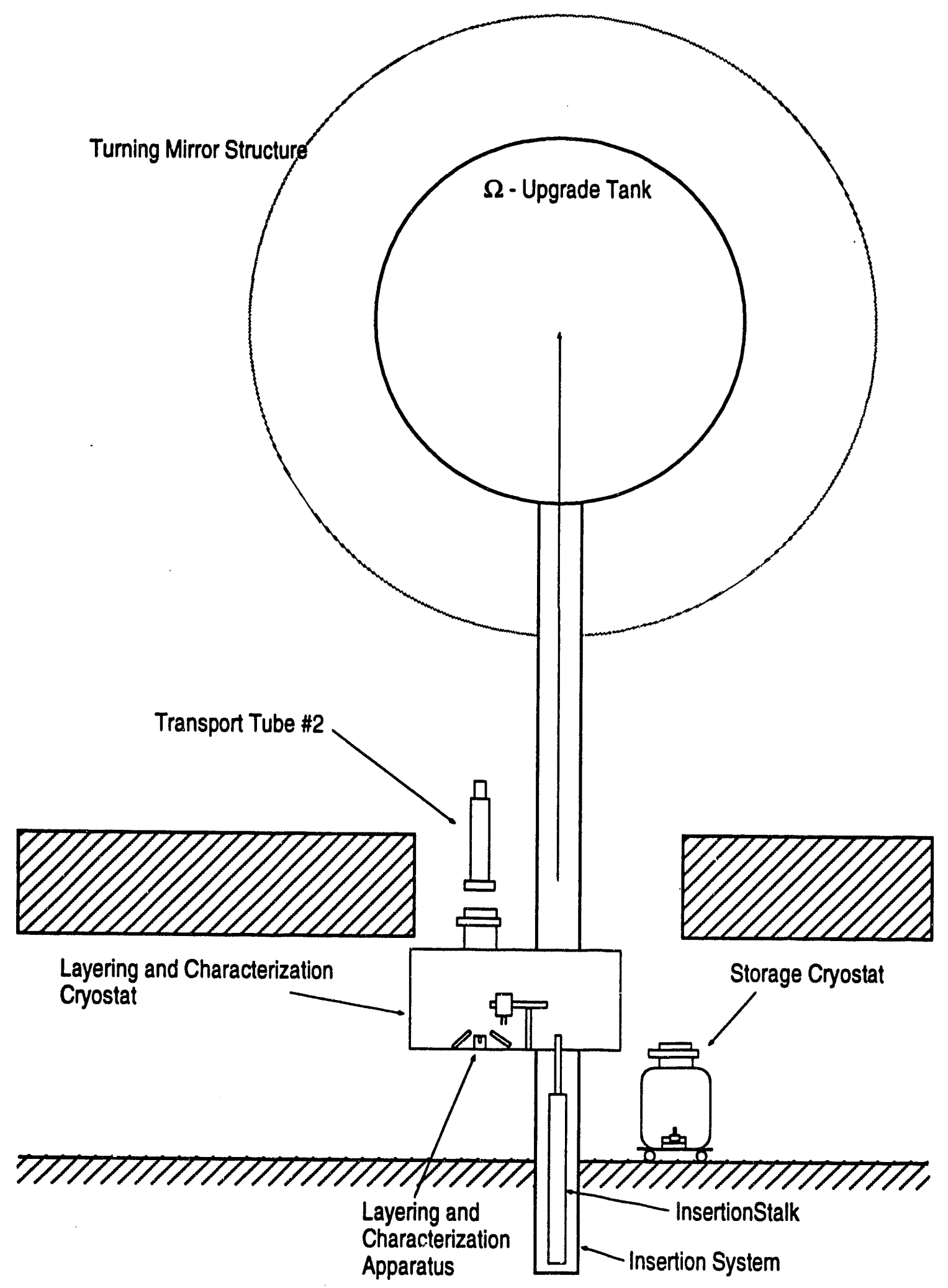

Figure 35: Layout of Target Delivery System. 


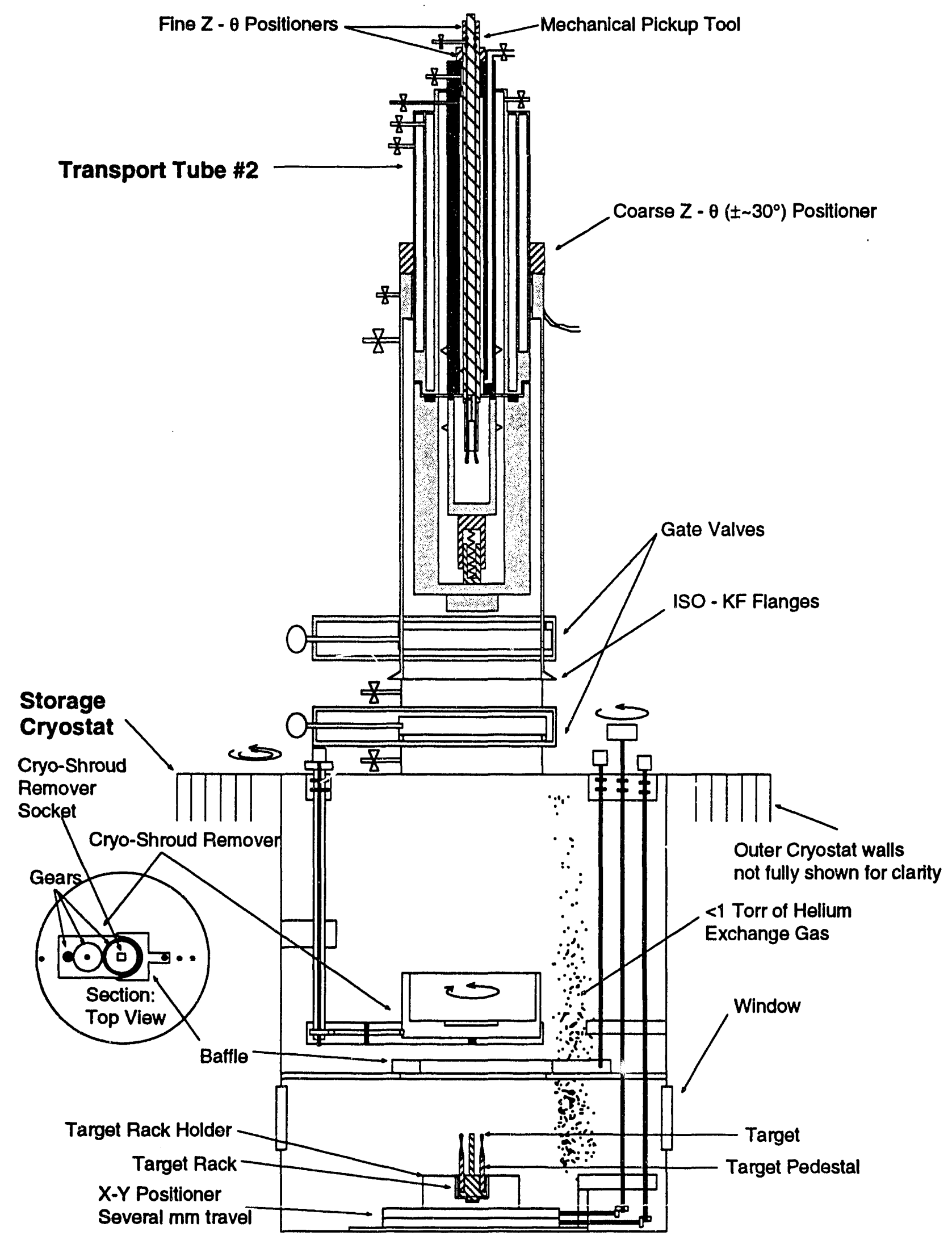

Figure 36: Transport tube \#2 is attached to the top of the storage cryostat. 


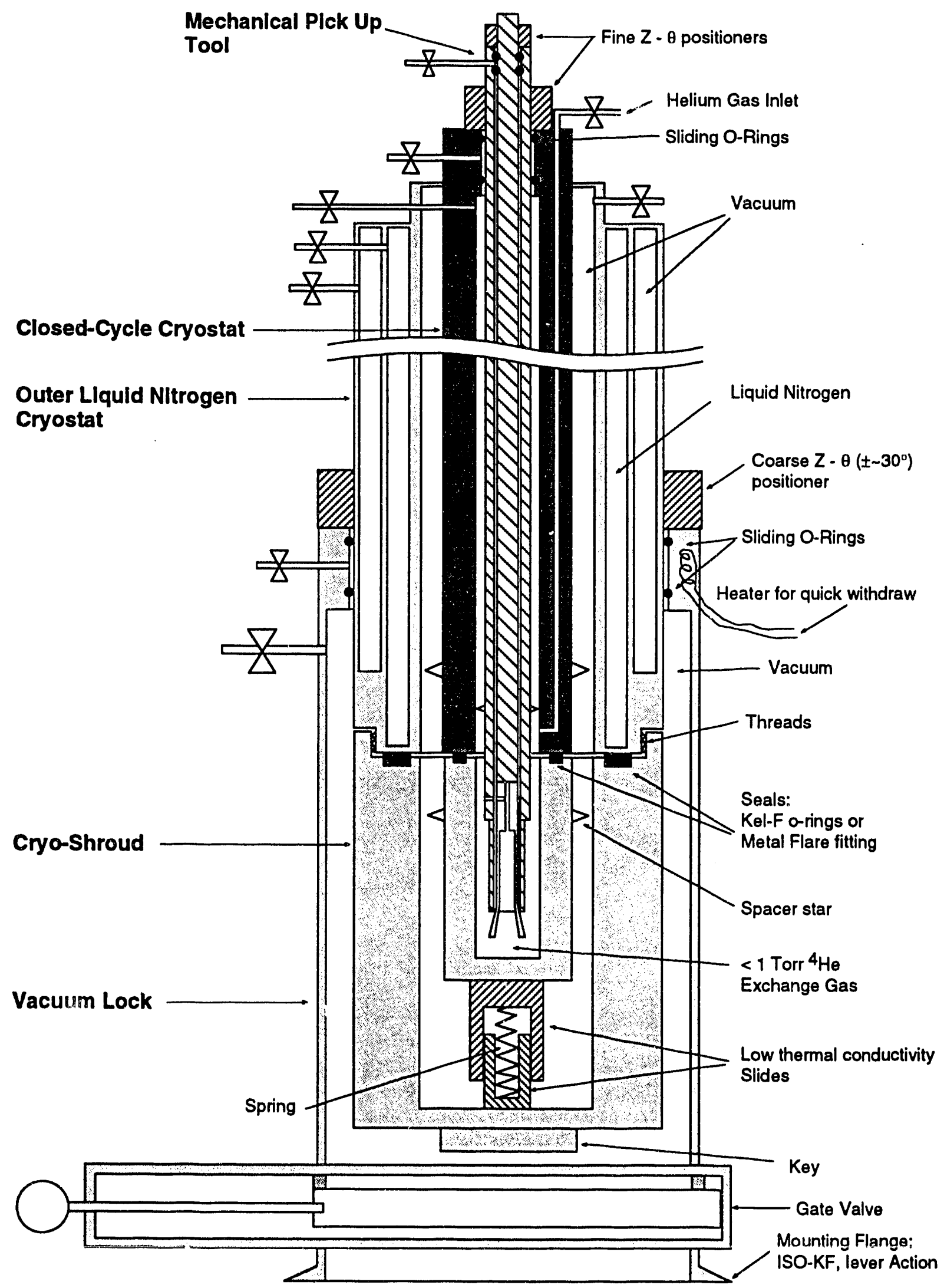

Figure 37: The basic design of transport tube \#2. 


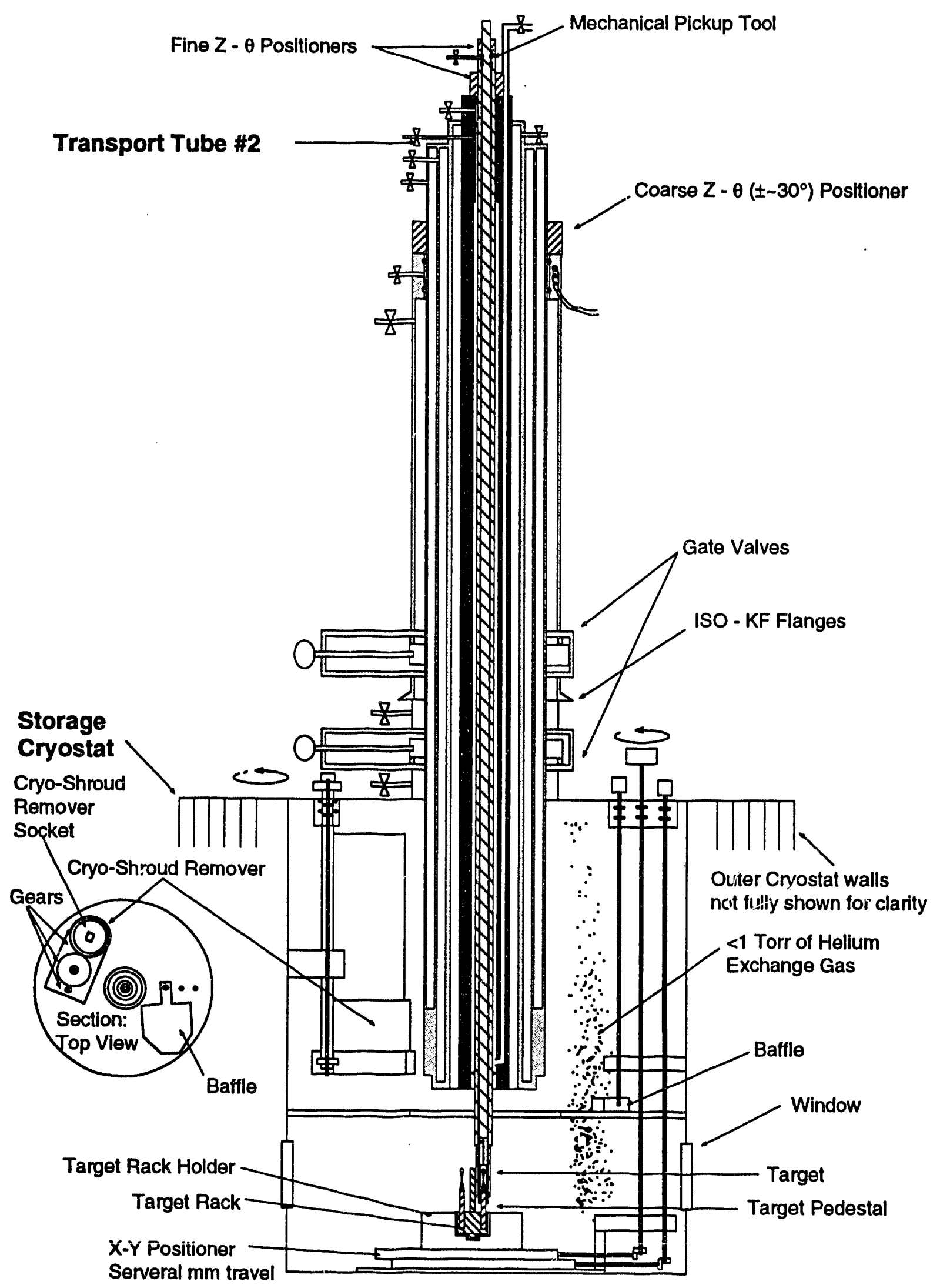

Figure 38: Transport tube \#2 is picking up a target from the storage cryostat. 


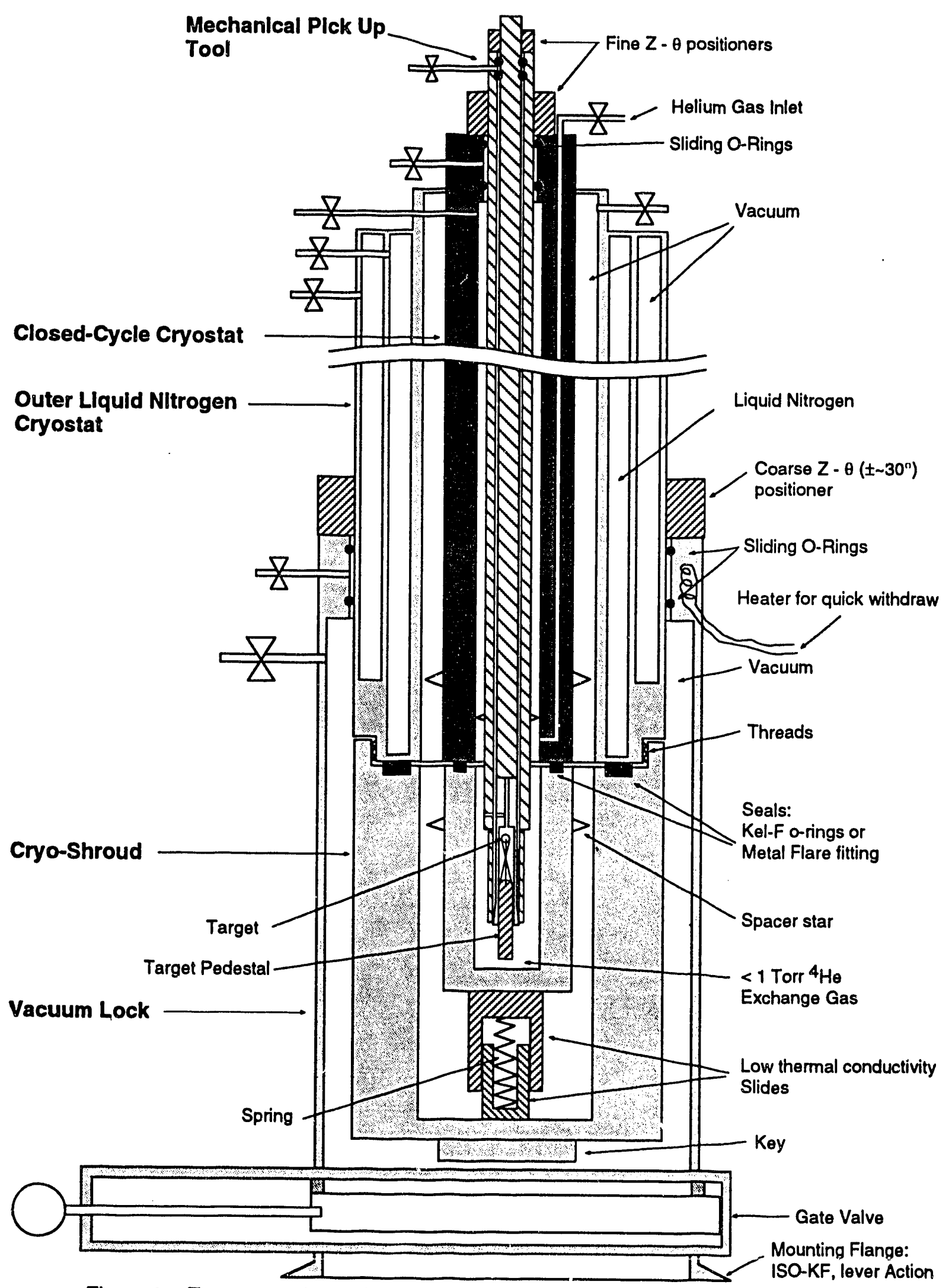

Figure 39: Transport tube \#2 is detached form the storage cryostat and is transporting a target to the layering cryostat. 


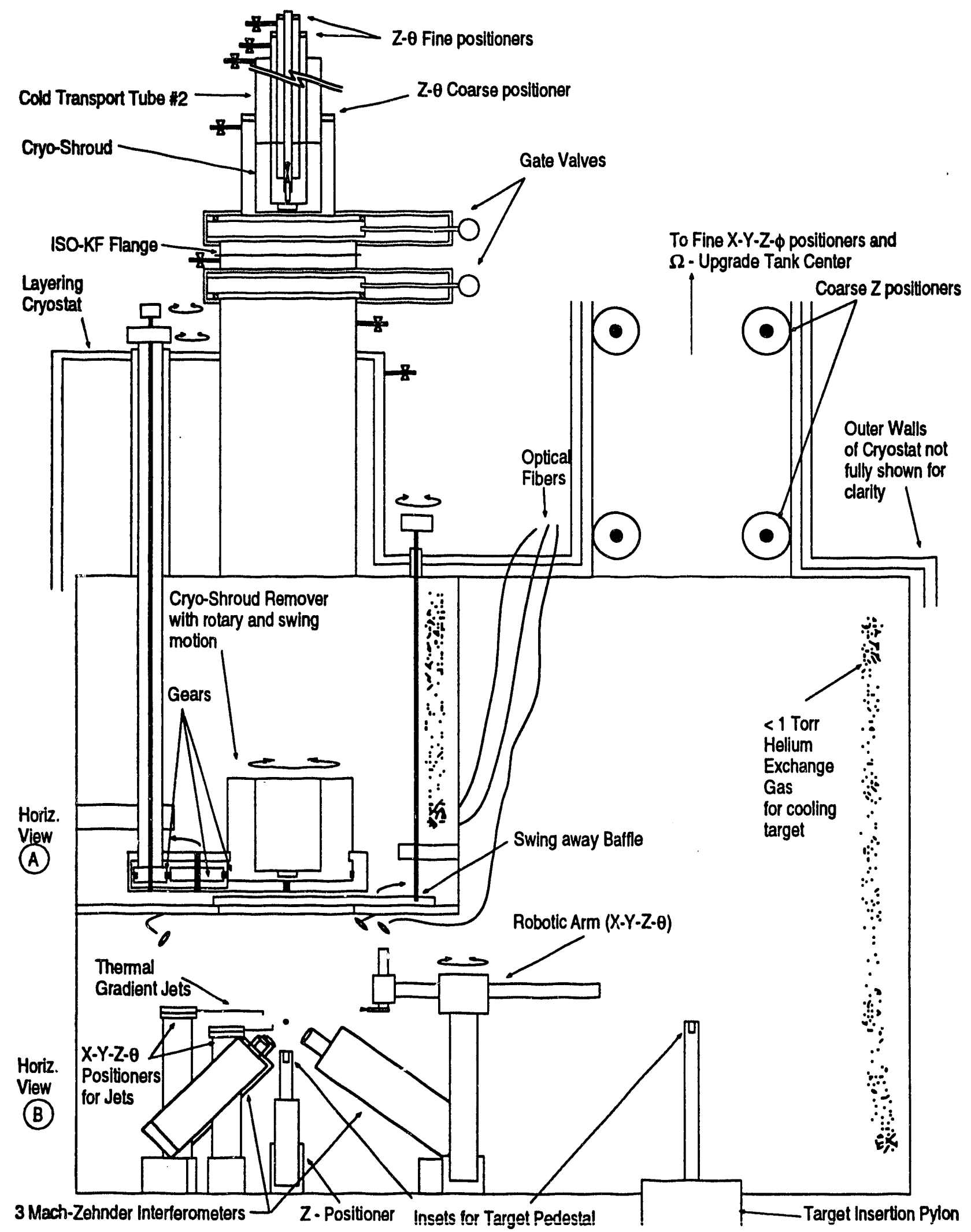

Figure 40: Transport tube \#2 is attached to the top of the layering cryostat. 


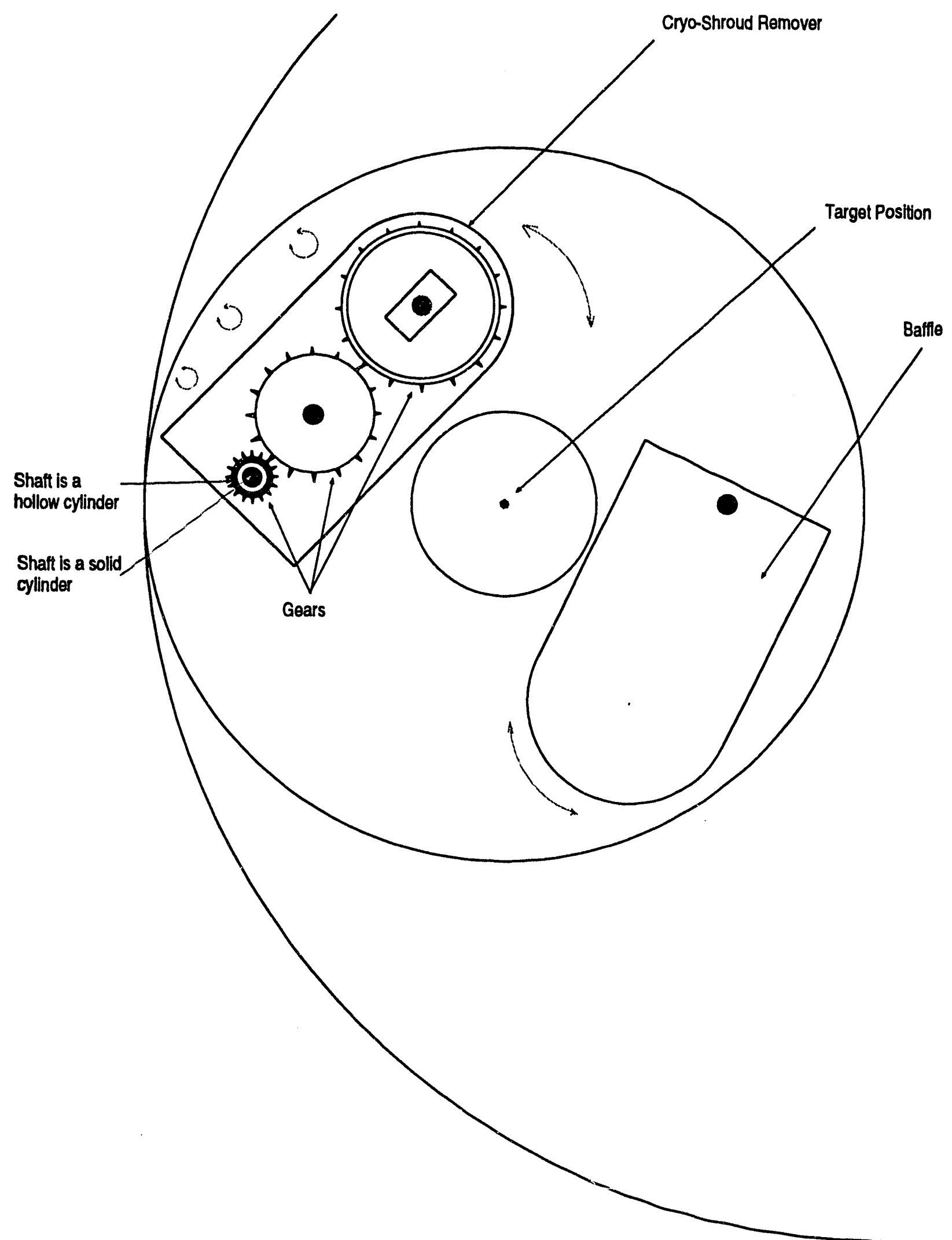

Figure 41: A horizontal section of the layering cryostat at the level of its cryo-shroud remover. 


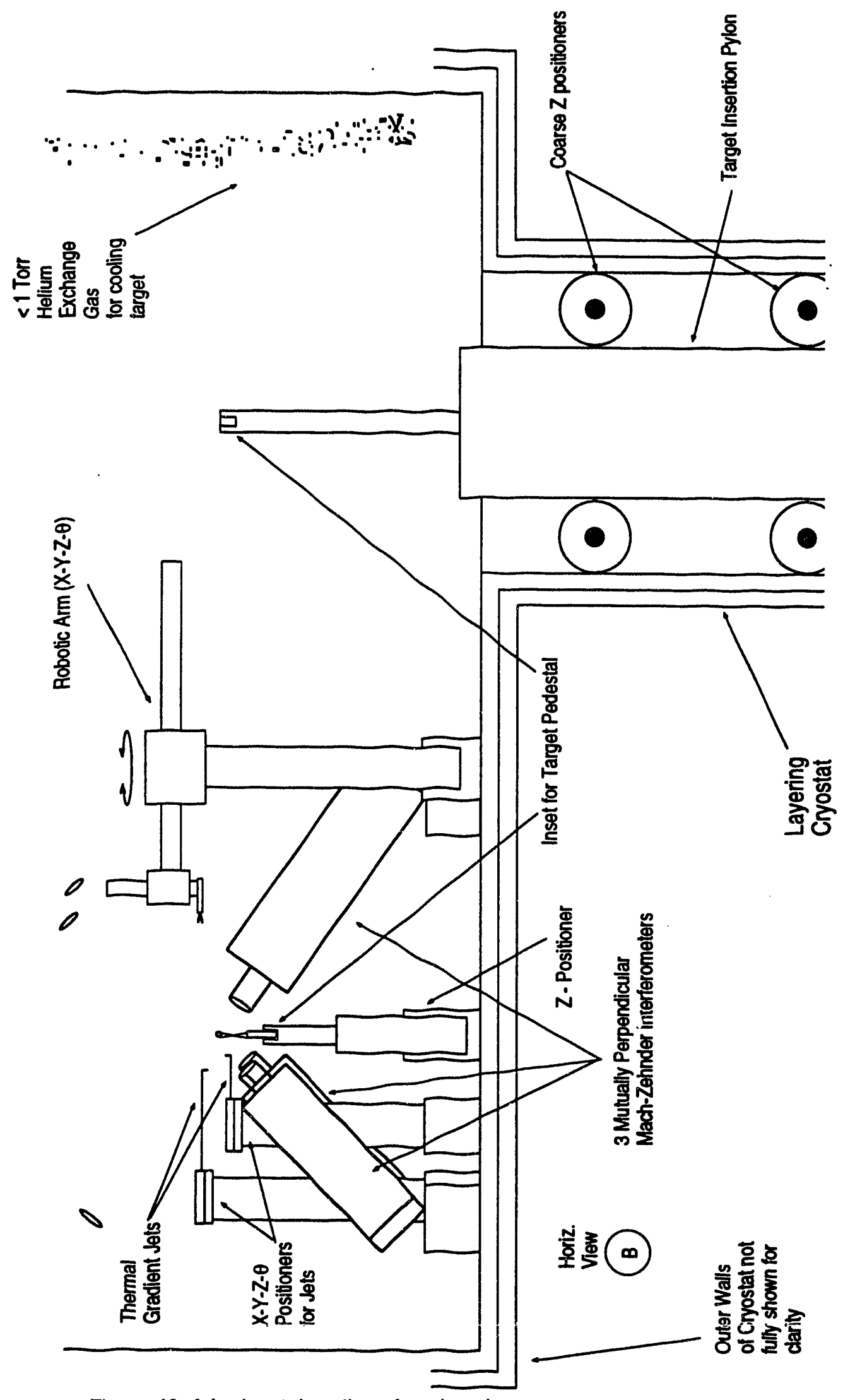

Figure 42: A horizontal section of the layering cryostat at its lower level. 

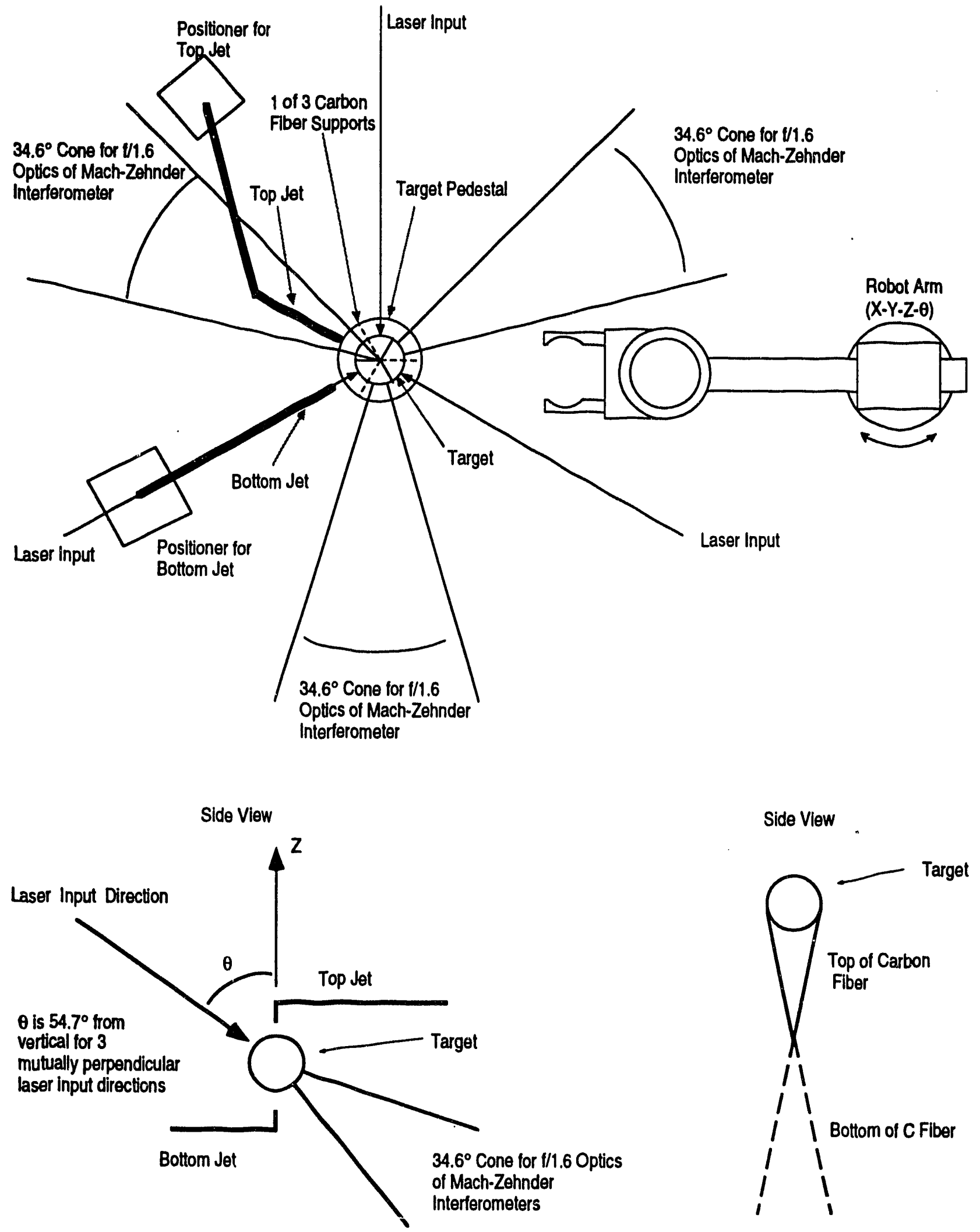

Figure 43: The placement of three mutually perpendicular interferometers, the thermal gradient layering jets, and the robot target transport arm are compatible. 


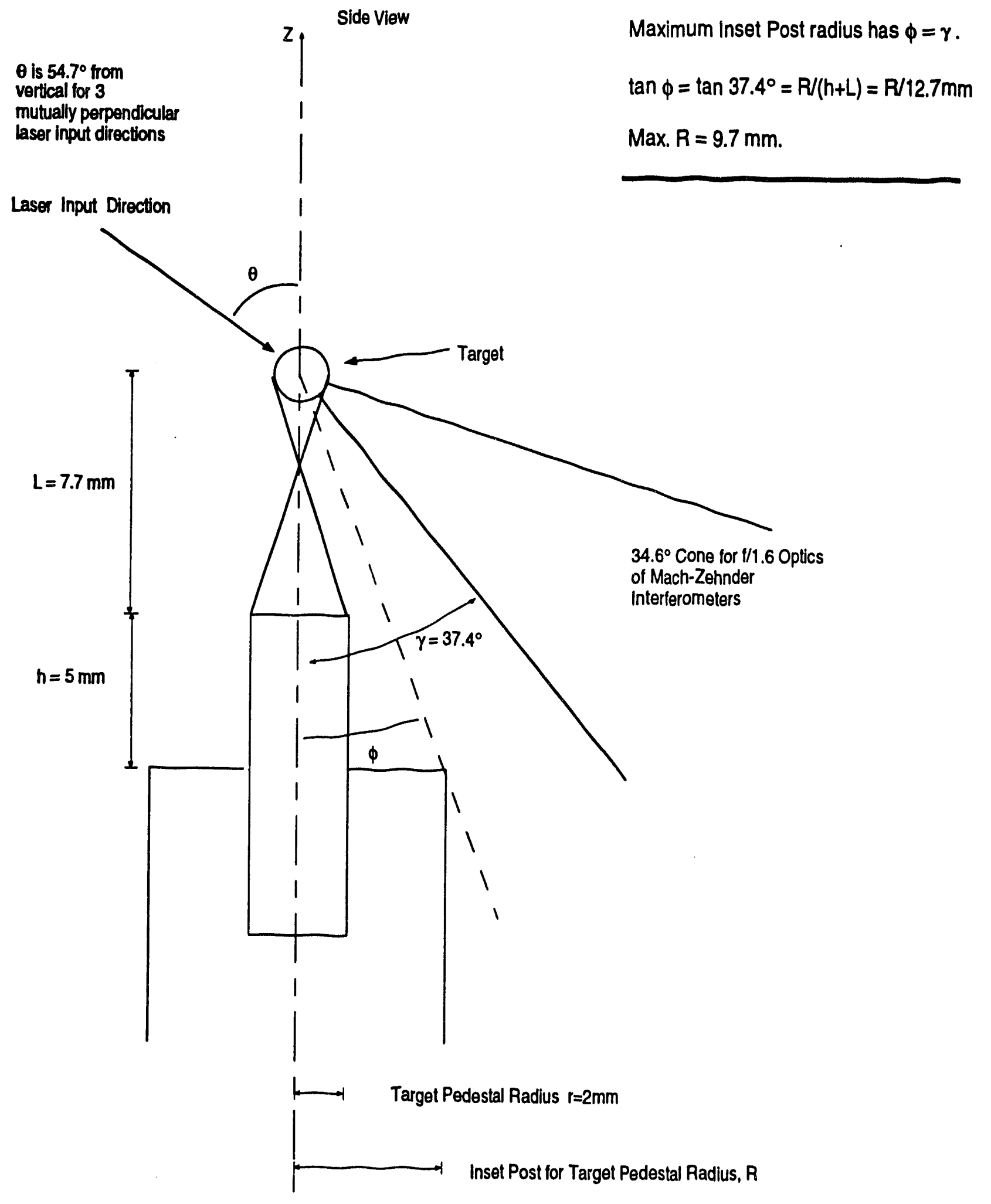

Figure 44: The maximum radius for the target inset post at the bottom of the layering cryostat is $9.7 \mathrm{~mm}$. 


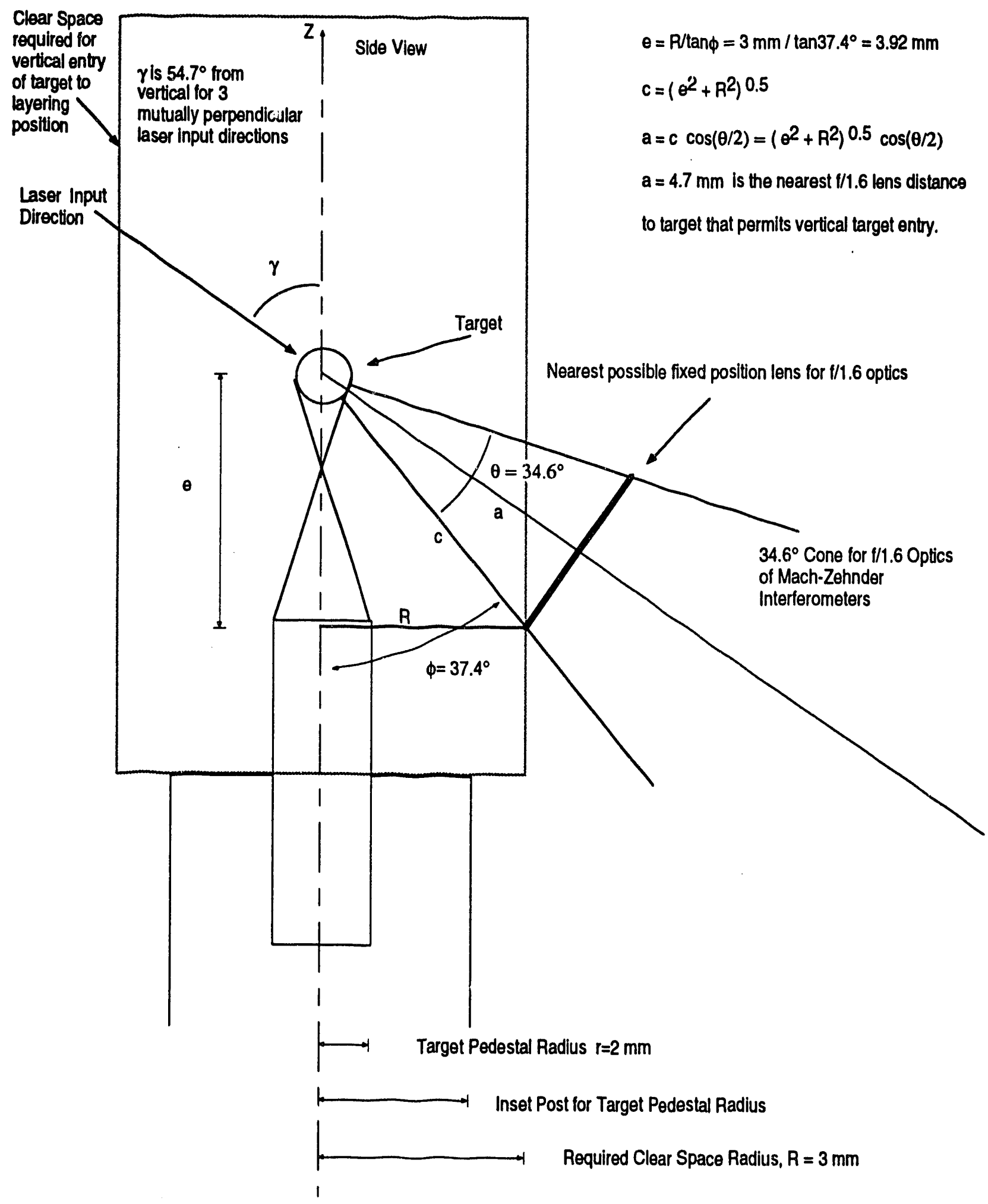

Figure 45: To prevent collision with transport tube \#2 while depositing a target into the layering cryostat, an $t / 1.6$ lens in the interferometer system cannot be closer that $4.7 \mathrm{~mm}$ to the target cent:r. 


\section{Attachment A \\ Design Requirements Report \\ University of Rochester \\ Cryogenic Target Delivery System}




\title{
DESIGN REQUIREMENTS REPORT UNIVERSITY Of ROCHESTER CRYOGENIC TARGET DELIVERY SYSTEM
}

\author{
by
}

R. L. FAGALY and D. N. BITTNER

Prepared under

DOE Contract No. DE-AC03-91SF18601

\section{GENERAL ATOMICS PROJECT 3896 \\ APRIL 1992}

\section{GEMERAL ATOMICS}




\section{Design Requirements Report University of Rochester Cryogenic Target Delivery System}

\section{Introduction}

The upgrade of the OMEGA laser at the University of Rochester's Laboratory for Laser Energetics will result in a need for large targets with thick uniform condensed layers of $D_{2}$ or DT and maintained at cryogenic temperatures. This mandates a cryogenic target delivery system capable of filling, layering, characterizing and delivering cryogenic targets to the OMEGA Upgrade target chamber. In addition, the development of a target delivery system for OMEGA could also benefit experiments at Lawrence Livermore National Laboratory in that the same technologies could be applied to NOVA, NOVA Upgrade or the future LMF. General Atomics has been assigned the task of developing the conceptual design for the cryogenic target delivery system. Construction and installation of the system will be part of a separate task. Design and fabrication activities will be closely coordinated with the University of Rochester and with both Lawrence Livermore National Laboratory (LLNL) and Los Alamos National Laboratory (LANL), drawing upon their knowledge base in fuel layering and cryogenic characterization. The program goal is to design, construct, and test the entire target delivery system by December 1995 . When completed (including an operational demonstration), the system will be shipped to Rochester for reassembly and commissioning in time for the OMEGA Upgrade cryogenic campaign, scheduled to start in 1997.

\section{Purpose of Document}

The purpose of this document is to describe the minimum requirements that are necessary and sufficient for a cryogenic target delivery system capable of delivering cryogenically layered $\mathrm{D}_{2}$ or DT targets into the OMEGA Upgrade target chamber. Design requirements that are the responsibility of the ICF National Laboratories or the University of Rochester are noted.

This Design Requirements Report will form the basis of the conceptual design of the OMEGA Upgrade Cryogenic Target Delivery System. We believe that a design can be developed that satisfies all the design requirements. However, it must be recognized that the design is not finalized. It is possible that certain requirements will prove to be incompatible with other requirements. Evaluation of the design against the design requirements will provide a mechanism for identification and resolution of any such incompatibilities.

It is anticipated that this document will be revised as the OMEGA Upgrade facility is finalized and constructed and as the target delivery system design matures. Modifications, additions or deletions will be made by the following procedure:

1) Requests for changes may be made by any member of the system design team (University of Rochester, LLNL, LANL or GAWJSA)

2) When the number and importance of requested changes justifies revision of this document, and when the system design team have agreed on resolution of the change, GA will prepare a revision for review by the GA project manager, the University of Rochester technical contact and the LLNL technical contact 
3) The revised document will then be submitted to the Lead Lab Technicai Coordinator and the Contracting Officer's Technical Representative for review and approval.

4) Upon approval, the revised document will then be numbered appropriately and issued.

There are modules (layering and characterization) of the target delivery system where the choice of technique to be used is still not decided. It is the responsibility of the ICF National Laboratories to specify these techniques and demonstrate that they are suitable for OMEGA Upgrade targets. Active research programs are currently underway at LLNL and LANL to develop these techniques. It is anticipated that an ICF community workshop will be held prior to December 1993 to make the choice of technique to be used. The Design Requirements Report will then be modified and reissued to reflect this decision.

\section{System Description}

The cryogenic target delivery system will be configured in modules: Target Support, Cryogenic Fill Station, Cryogenic Storage, Transport, Layering, Characterization and Target Insertion. Suitable plastic target shells will be supplied by GA. These targets will be mounted, filled with appropriate fuel gases, cryogenically cooled and transported into a module that will create a uniform liquid/solid fuel layer. They will then be characterized (perhaps simultaneously with the layering system) and transported into the target chamber. Pre-shot characterization will confirm proper target positioning and, if needed, verify layer uniformity. A cryogenic storage system will also be developed to allow for inventory purposes and (possibly) for off-site transport of filled targets. A flow diagram of the process is shown in fig. 1.

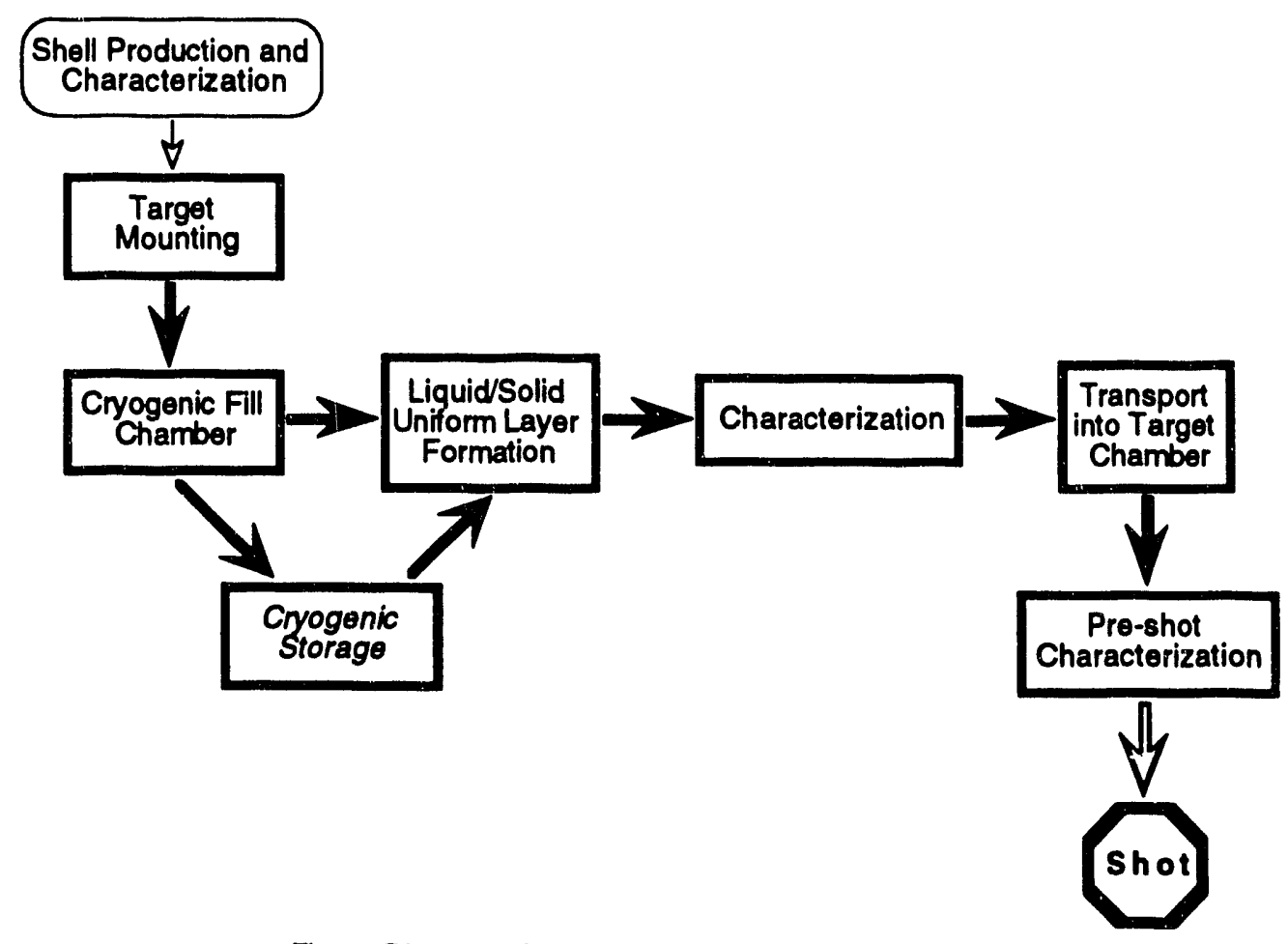

Fig. 1. Diagram of Cryogenic Target Delivery System. 


\section{System Design Requirements}

4.1. Timeline

OMEGA is scheduled to shut down operations in October 1992. OMEGA Upgrade is scheduled to start operation by 1995 , with the first cryogenic target campaign planned for 1997. In order to meet this schedule, it will be necessary to demonstrate operation of the target delivery system at GA by the end of 1995 . Since cryogenic shots are scheduled to start in the second half of 1997, the system must be installed at Rochester and fully integrated and operational by the middle of 1997.

Design Requirements Report Due:

Baseline Concept Definition Due:

Concept Definition Review Completed:

Specification of Cryogenic Target Layering and Characterization Techniques:

Target Delivery System Testing Completed:

Systems Demonstration at Rochester:
April 30, 1992

July 31,1992

September 30, 1992

December 31, 1993

December 31, 1995

June 30, 1997

This schedule is based on the University of Rochester's estimate that the OMEGA Upgrade cryogenic campaign will start in 1997. This schedule requirement poses severe demands on the schedule for system design, construction and licensing. Licensing for tritium operation is expected to be a pacing item and will require early completion of design activities. This will dictate the pace and level of effort of the design activity, and will receive serious study early in the conceptual design task.

The technology for layering and characterizing liquid and/or solid $D_{2}$ and DT is not sufficiently developed. This is a major area of uncertainty in the program. We have assumed Livermore and/or Los Alamos will transfer all the layering and characterization technology required for $\mathrm{D}_{2}$ or DT-filled CH-based polymer targets by December 31, 1993.

\subsection{Unfilled Shell Characteristics}

Although shell production is not part of this task, the shells used must satisfy specific criteria (sphericity, wall thickness and concentricity, surface finish, etc.). Shells will be procured from normal target fabrication production runs. It may be necessary to modify these production shells to meet the requirements of a cryogenic target (e.g., opaque shells to riveet the need for an optical shine-through barrrier for $351 \mathrm{~nm}$ light). The physical properties of the polymer(s) used will be important in determining permeation fill rates and final pressures. The physical properties (e.g., yield strength, permeability, radiation damage) of the polymer(s) used will be important in determining DT and $D_{2}$ fill rates and pressures. Should opaque targets be used, the coating(s) properties with respect to hydrogen permeation must be considered. Also, before tritium is added, the effects of radiation damage on these properties will have to be assessed. Once filled, the assembly is referred to as a target. 
4.2.1. The target delivery system will utilize $700-1100 \mu \mathrm{m}$ diameter $\mathrm{CH}$-based polymer shells filled with $D_{2}$ or DT.

4.2.2. The shell wall thickness shall be $5-10 \mu \mathrm{m}$.

4.3. Target Support

Current OMEGA targets $(\sim t \mathrm{~mm}$ diameter) are supported between spiderwebs, and glued in place with a thin coating of parylene. The proposed cryogenic targets will be about $4 x$ larger, and contain 50-100x as much fuel as present targets. Ideally, a stalk or pendulum mount is preferable; this requires development of a glue that will survive thermal cycling. Until such a mounting method is developed, the baseline mounting method will be Rochester's crescent and spiderweb. In any case, the target mounting method must not cause a major perturbation on surface finish. Additionally, the targets must be kept refrigerated after gas filling; this places new restrictions on access to the target. Because of the restricted access to cryogenic targets (especially DT-filled), the target will be attached to the support (holder) before gas filling. The target delivery system will mount the targets on a target support such that:

4.3.1. The target mounting method must be thermally and mechanically stable to better than $\pm 5 \mu \mathrm{m}$ dimensionality.

4.3.2. The target mount must not interfere with any of the 60 OMEGA Upgrade laser beams or associated diagnostics.

\subsection{Cryogenic Fill Station}

High pressure fills in polymer capsules require maintaining shells at cryogenic temperatures after filling to prevent the shells from bursting. This, combined with the requirement that the gas permeate into the shells, implies that the system must work in a temperature range from room temperature down to as low as liquid helium temperatures. It is assumed that no targets will require overcoatings that are significantly less permeable to $D_{2}$ or DT than that of the $\mathrm{CH}$-based polymer material used to make the shells. Factors that affect the design of the fill station include requirements for target throughput and tritium inventory capacity. The fill station must be designed to the highest environmental safety and health standards.

4.4.1. The target delivery system shall include the ability to fill empty targets with $D_{2}$ or $D T$ gas to pressures greater than $1000 \mathrm{~atm}$. at room temperature.

4.4.2. The target delivery system shall include the ability to cool filled targets to below the triple point of the filled gas without rupturing the filled target.

4.4.3. The tritium inventory of the DT fill station shall be less than 1 gram of tritium, but sufficient to fill at least six months supply of targets.

4.4.4. The DT fill station must not release any tritium to the environment. 
4.4.5. The DT fill station must contain a backup system for tritium recovery.

4.4.6. The DT fill station must include power failure protection.

\subsection{Cryogenic Storage}

A storage system is not an absolute necessity for a cryogenic target delivery system. However, it can be very useful in that it would allow the accumulation of a stockpile of targets. This target inventory would act as a buffer, so that the production rate could be somewhat independent of the target usage rate. The storage system could also be designed to allow cryogenic targets to be transported off site. The rate of ${ }^{3} \mathrm{He}$ buildup will place constraints on the amount of time a DT-filled target may be stored. The projected rate of target usage will determine the size of the inventory to be maintained. The physical properties of the polymer(s) used in target construction will affect the design of the storage system. These properties will determine, at least in part, the storage temperature and the possible duration of storage.

4.5.1. If a cryogenic storage system (for filled targets) is used, it must be able to withstand a complete loss of refrigerant without a release of tritium to the environment.

4.5.2. If used, the cryogenic storage system must be able to hold at least six filled targets.

4.6. Transport

Filled targets must be maintained at cryogenic temperatures. The target transport systems must move a mounted target from the DT-fill chamber and transport it between the DT-fill system, cryogenic storage, layering, characterization and target insertion systems. Transport methods must not cause temperature excursions that would degrade the quality of the layered fuel.

4.6.1. Temperature excursion: in the environment surrounding the layered target shall not exceed $\pm 0.2 \mathrm{~K}$.

4.6.2. In the situation when a filled target accidentally bursts, the transport method must not allow a release of tritium to the environment.

\subsection{Layering}

The layering system is a key element in the target delivery system. The ICF National Laboratories have the responsibility of specifying the method that will be used to create the uniform cryogenic fuel layer. Depending on the type of system chosen (Beta layering, differential thermal heating, fast refreeze, etc.), system characteristics may vary. Our plan is to treat the layering system as a detachable element which, when completed, is inserted into a section of the target delivery system. Design features and requirements are to be supplied by the ICF National Laboratories.

We will monitor laboratory progress in layering techniques $\left(D_{2}\right.$ and $\left.D T\right)$. After the layering 
technique is decided upon, a design will be developed and submitted to both Rochester and the national laboratory responsible for the chosen layering technique. Upon approval of the design, the layering module will be constructed, tested and integrated into the rest of the system. Because characterization is integral to any layering technique, design and construction activities will be coordinated with the characterization group. Specific requirements as to the layering method are as follows:

4.7.1. The target delivery system will use a layering method(s) to be determined.

4.7.2. The layering method(s) shall produce uniform liquid and/or solid $D_{2}$ or DT layers ranging between 10 and $100 \mu \mathrm{m}$.

4.7.3. The design goal of the layering method will be to produce an inner surface finish better than $1000 \AA$ with fuel mass uniformity, concentricity and sphericity all better than $2 \%$.

4.7.4. The layering method must maintain the fuel layer characteristics until the target is shot.

\subsection{Characterization (at cryogenic temperatures)}

It will be important to have sufficient resolution in a characterization system to determine nonuniformities in the transparent capsules. This is also important in the cryogenic fuel layers. Some overlap of characterization techniques should be possible. Should opaque targets be used, an appropriate characterization method must be developed in parallel to this task. Final pre-shot characterization will be needed to confirm proper location of the target in the target chamber. It may be necessary to perform a final check on the shape of the fuel layer in the target chamber to confirm the integrity of the layering process during transport to the target chamber.

Like the layering method(s), development of characterization techniques is an ICF National Laboratory task. If the current characterization system used at Rochester is adaptable to OMEGA Upgrade, an analysis of the system must be made and appropriate modifications done. If the characterization system is not suitable, then alternative approaches must be considered, with direction coming from the national laboratories. Specific items relating to characterization are as follows:

4.8.1. The target delivery system will use a characterization method to be determined.

4.8.2. The characterization method must not deform the fuel layer until the target is shot.

4.8.3. The characterization method must verify fuel homogeneity.

4.8.4. The goal of the characterization method is to provide information on surface roughness to $\pm 1000 \AA$.

4.8.5. The characterization method must provide information on fuel layer thickness, concentricity and sphericity at the $1 \%$ level. 
4.8.6. The final pre-shot characterization in the target chamber must determine the location of the target to $\pm 5 \mu \mathrm{m}$ and confirm the integrity of the fuel layer (if necessary).

\subsection{Target Insertion}

Targets will need to be positioned at the focus of the OMEGA upgrade laser system. In addition, they must be maintained at cryogenic temperatures until the laser system fires. This may require the use of cold helium gas jets and/or a retractable cryogenic shroud. The system must also allow for precise target positioning and characterization within the target chamber and must be highly integrated with the target transfer module. Operation with DT targets will preclude human access to the target chamber immediately after a shot.

4.9.1. The target insertion system must be compatible with the 60 OMEGA Upgrade laser beams and associated diagno.stics.

4.9.2. The target chamber insertion system for the OMEGA Upgrade laser must have an interlock to prevent the laser from firing if there is any possibility of damage to the laser or optics.

4.9.3. The target insertion system must have greater than $\pm 5 \mathrm{~mm}$ positioning travel at the laser focus.

4.9.4. The target insertion system must have better than $\pm 5 \mu \mathrm{m}$ positioning accuracy at the laser focus.

4.9.5. The target insertion system must maintain the target to better than $\pm 5 \mu \mathrm{m}$ prior to the laser shot.

4.9.6. Any refrigeration technique(s) that cool via gas conduction must not allow the gas pressure to exceed $10^{-4}$ torr at the tank center at the time of the laser shot.

4.9.7. The target insertion system must be able to cycle targets in less than two hours.

\subsection{Safety and Environmental Requirements}

Environmental safety and health issues are of utmost importance. They will be taken into consideration at all times. This includes determination of safety and tritium issues for each module.

4.10.1. The target delivery system must meet all local, state and federal safety and environmental requirements, especially those related to the handling of radioactive materials.

4.10.2. The total tritium inventory in the target delivery system shall not exceed 1 gram.

4.10.3. No system or subsystem shall allow the release of tritium to the environment. 

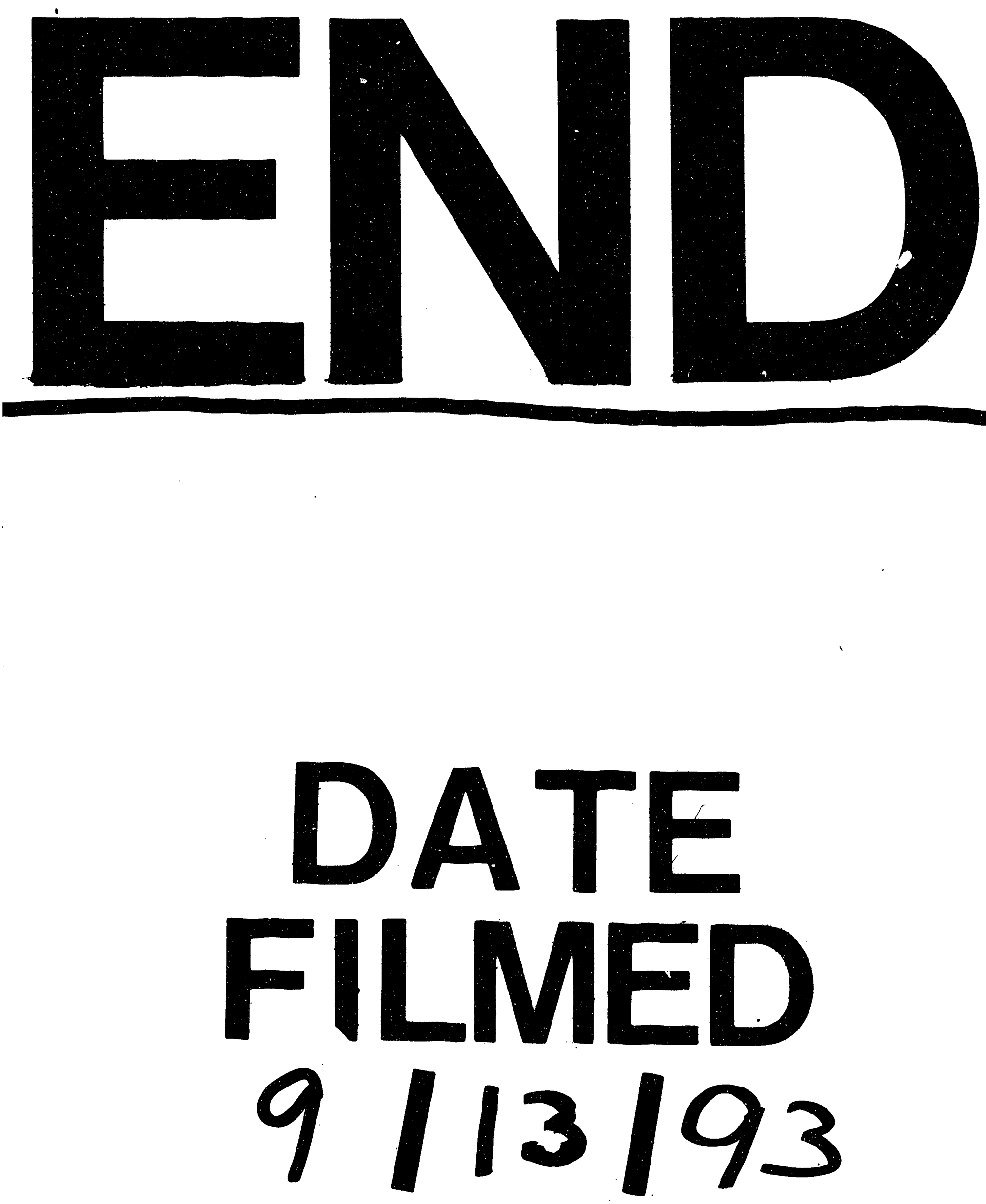
